Preprint typeset in JHEP style. - HYPER VERSION

hep-th/0310001

CPHT-RR 090.0903

\title{
D-branes in Standard Model building, Gravity and Cosmology
}

\author{
Elias Kiritsis \\ CPHT, UMR du CNRS 7644, Ecole Polytechnique, \\ 91128, Palaiseau, FRANCE \\ and \\ Department of Physics, University of Crete \\ PO Box 2208, 71003 Heraklion, GREECE \\ E-mail: kiritsis@cpht.polytechnique.fr
}

\begin{abstract}
D-branes are by now an integral part of our toolbox towards understanding nature. In this review we will describe recent progress in their use to realize fundamental interactions. The realization of the Standard Model and relevant physics and problems will be detailed. New ideas on realizing 4-dimensional gravity use the brane idea in an important way. Such approaches will be reviewed and compared to the standard paradigm of compactification. Branes can play a pivotal role both in early- and late-universe cosmology mainly via the brane-universe paradigm. Brane realizations of various cosmological ideas (early inflation, sources for dark matter and dark energy, massive gravity etc) will be also reviewed.
\end{abstract}

To appear in Physics Reports 


\section{Contents}

1. Introduction and perspective 2

2. A survey of various string theory compactifications 6

2.1 The heterotic string 6

2.2 The type-I string 7

2.3 Type II string theory 9

3. Orientifold vacua, chirality and supersymmetry 10

3.1 D-brane mechanics 11

3.2 D-branes at orbifold singularities 12

3.3 Internal magnetic fields and branes intersecting at angles 14

3.4 Intersecting $D_{6}$-branes 16

3.5 Supersymmetry breaking 17

3.5.1 Supersymmetry breaking in low-string scale orientifolds 18

4. Anomalous U(1)s in six and four dimensions and their role in string model building

4.1 Anomalies and symmetries in four dimensions 21

4.2 Anomalies in six dimensions 23

4.3 String calculation of anomalous U(1) masses 26

4.4 An example: anomalous $\mathrm{U}(1)$ masses in the $Z_{6}^{\prime}$ orientifold 28

4.5 Physical axions 29

5. D-brane Standard Model building 32

5.1 Symmetries and Charges 34

5.2 Gauge couplings 37

5.3 Viable D-brane configurations 39

5.4 The quark and lepton mass structure 43

5.5 The Standard Model structure from intersecting D-branes 47

6. Novel realizations of four-dimensional gravity 49

6.1 Randall-Sundrum localization 49

6.2 Brane Induced Gravity 52

6.3 Randall-Sundrum meets Brane-Induced Gravity 57

6.4 Graviton emission in the bulk and brane energy-loss 59 
7. The brane-universe and its cosmology 62

7.1 Brane cosmological evolution in the Randall-Sundrum paradigm. 62

7.2 How Brane-induced gravity affects the cosmological evolution 66

7.3 Brane-bulk energy exchange 68

7.4 Phenomenology of brane-bulk energy exchange and its impact on brane cosmology 70

7.4.1 The cosmological equations for brane-bulk energy exchange 72

7.4 .2 The four-dimensional regime 73

7.4 .3 The exact solution for RS outflow 74

7.4.4 Inflating fixed points 76

7.4.5 Tracking solutions 78

7.4.6 Fixed points in the non-linear regime 79

7.4.7 Other accelerating solutions 80

7.5 Mirage Cosmology 81

7.5.1 Probe-brane geodesics and induced cosmology 84

7.5.2 Orbits, bounces and static universes 88

7.5.3 A partial survey of various induced mirage cosmologies 89

7.5.4 Near-horizon region 92

7.5.5 Static universe solutions 92

7.5.6 The Hyper-universe 94

7.6 Brane/antibrane systems and inflation 96

7.7 The cosmology of massive gravity and late time acceleration 98 7.7.1 Cubic interactions 102

7.8 Massive gravity and gauge theory 103

8. Acknowledgements 108

\section{Introduction and perspective}

The basic problem in particle physics is to extend our knowledge of fundamental physics beyond the energies so far explored by accelerators and other experiments. The Standard Model of physics, the culmination of twentieth century research in the high energy frontier, has been confirmed to a great degree of accuracy in many experiments. Despite the fact that the Higgs particle remains experimentally elusive, few doubt that there may be major surprises in this direction. It is fair to say that it remains to be decided whether the Higgs is a fundamental scalar or a bound state, and what some of its parameters are. 
In a related direction, there is concrete experimental evidence consistently indicating that neutrinos have (tiny) masses and mixings and the SM should be extended to accommodate this. Several ideas on how this can be done, have been forwarded many years ago and we are currently awaiting for experiment to decide.

On the other hand there are two theoretical issues that since the late seventies have made physicists believe that the SM, although extremely successful in describing current experimental data, cannot be the final story.

(A) (Quantum) gravity is not incorporated.

(B) The SM suffers from the hierarchy problem, namely, that some low energy scales like the mass of the Higgs are technically unnatural if the SM is extended to energies much beyond the present energy frontier.

There may be further questions concerning the explanation of the values of the parameters of the SM but, although important, they are not as central as the ones above.

To these issues we should add some novel questions coming from the spectacular progress in the last twenty years of observational cosmology, which can no more be ignored:

(C) What and how has made the universe inflate in its early stages?

(D) What comprises the dark matter of the universe?

(E) Why the present day cosmological constant scale (or dark energy) is of the same order of magnitude as the Humble constant and many orders of magnitude smaller than the natural scale of four-dimensional gravity, $M_{P}$ or other fundamental particle physics scales?

It is felt by many physicists, that the previous questions are most probably interrelated.

The adventure of grand unification in the late seventies/early eighties followed a path, extending the successful ideas of non-abelian gauge symmetry that led to the SM description of the fundamental interactions to its natural conclusion. In this context, the natural scale of the unified theory turned out to be $M_{U} \sim \mathcal{O}\left(10^{16-17}\right) \mathrm{GeV}$ which is not far from the Planck mass $M_{P} \sim 10^{19} \mathrm{GeV}$ where quantum gravitational effects could no more be ignored. Moreover, a new question surfaced:

$\left(\mathbf{B}^{\prime}\right)$ Why $M_{U}$ is so close to $M_{P}$ ?

In addition, the issue of question (B), became acute: the theories are technically useless due to the instability induced by the hierarchy problem.

Several ideas were put forward to deal with the large hierarchy of scales. The minimal one, technicolor, despite many attempts so far has failed to produce satisfactory models, mostly because it invokes strong coupling physics of non-abelian gauge theories that makes such theories complicated and not very predictive.

An independent idea, involving a new symmetry, supersymmetry, had more success, at the expense of introducing new degrees of freedom. Since the low energy world is not supersymmetric, if supersymmetry is responsible for the stability of the 
fundamental theory, it must be spontaneously broken at low energy. Mechanisms for this are known, but it is fair to say that no complete and successful model exists so far.

However, physicists today more than ever believe that supersymmetry in some form holds the key to the successful resolution of the hierarchy problem and if so, its avatars should be visible in the next round of accelerator experiments. Moreover, this belief was strengthened by string theory which eventually come into the arena of fundamental interactions.

It soon became obvious that spontaneously broken global supersymmetry, although technically enough to stabilize grand unified theories, is too constrained to describe the low energy data. The advent of local supersymmetry allows such constraints to be weakened but brings gravity back in the game. Gravity is an integral part of a locally supersymmetric theory (supergravity) and this opens the new pandora's box of quantum gravity: the theory is non-renormalizable and the issue of stability (quadratic divergences) resurfaces.

String theory, after a short lived stint as a theory of hadrons, regained popularity, because it was (and still is) the only theory that provides a workable theory of quantum gravity (at least in perturbation theory and for energies below the Planck scale). It provided a new theoretical argument for supersymmetry: the presence of space-time fermions in the theory implies an underlying supersymmetry.

In particular, heterotic string theory, provided a framework that incorporated the successes of grand unification, supersymmetry, and a controllable treatment of quantum gravity and justifiably monopolized the attention of high-energy physicists for a decade.

The heterotic string approach to the fundamental interactions [1], provided an answer to the question $\left(\mathbf{B}^{\prime}\right)$. It predicts that the unification scale is close to the four-dimensional Planck scale.

Model building in the context of the heterotic string has given several models which at low energy come close to the standard model [2, 3]. However, although the setup is very appealing, there are still unresolved problems in this context.

- Semi-realistic heterotic ground-states contain particles with fractional electric charges (other than the quarks). Strong coupling dynamics in the hidden sector is advocated in order to bypass this problem.

- $\mathrm{N}=1$ supersymmetric vacua have an involved structure and the solutions to the D- and F-flatness conditions are complicated.

- The mechanism of supersymmetry breaking is not well understood. There are two candidate mechanisms: Gaugino condensation can be argued to happen in semi-realistic heterotic vacua, breaking supersymmetry. It involves, however, strong coupling dynamics and because of this, it has so far failed to have a quantitative description. Moreover, it is not known (modulo some partial recent progress using 
non-perturbative dualities) how to implement it beyond the effective field theory level.

An alternative mechanism of supersymmetry breaking which is geometrical and can be implemented at the full string theory level is the Scherk-Schwarz mechanism, which was introduced earlier in higher dimensional supergravity, [4], and was subsequently implemented in string theory [5]-8]. The supersymmetry breaking scale is proportional to an inverse compactification length. However, in the context of simple (orbifold) compactifications of the heterotic string all compactification scales are of the order of the string scale $M_{s}$ which is of the same order as the four-dimensional Planck scale. It is thus difficult to generate a supersymmetry breaking scale of a few $\mathrm{TeV}$, necessary for the successful resolution of the hierarchy problem.

- The hierarchy of SM fermion masses is not easy to generate. There are ideas in this direction using higher-order terms in the tree-level potential and strong coupling dynamics in the hidden sector, but no complete model exists.

- There is a generic axion problem associated with many semi-realistic heterotic vacua.

Despite these shortcomings, the heterotic string is an elegant candidate for describing nature and may provide further breakthroughs in this respect.

In the last decade, further progress in the understanding of string theory indicated that other ten-dimensional supersymmetric string theories (type-IIA/B closed strings and type-I closed and open strings) should be treated on the same footing as the heterotic theory. The other string theories till then had been handicapped because IIA/B string theory could not accommodate the SM fields in the perturbative spectrum [9], and the type-I theory had an intricate and little-understood structure [10]-[16]. It was successfully argued [17, 18] that the five distinct ten-dimensional supersymmetric string theories can be viewed as vacua of a larger (unique?) theory, coined M-theory. Moreover, non-perturbative dualities relate the strong coupling behavior of a given theory to another weakly coupled string theory or a still elusive eleven-dimensional theory.

A key element in these non-perturbative dualities was the recognition that extended solitonic-like objects (D-branes and NS5-branes) form an integral part of the theory. Moreover, D-branes were shown to have a simple weak-coupling description as defects where closed strings can open up 19. A detailed understanding of their fluctuations ensued, which geometrized gauge theories and provided new vistas in the relationship between gauge theory and gravity/string theory [20, 21] with a interesting reconsideration of the strong coupling dynamics of gauge theories. An important byproduct was that D-brane degrees of freedom could explain the macroscopic entropy of black holes [22, 23] and made plausible that a unitary description of their physics may be at hand.

These developments enlarged substantially the arena for the search of SM-like vacua in string theory. In novel contexts, D-branes play a central role, and the 
purpose of the present lectures is to discuss their role in new avenues for SM building, as well as to new realizations of four-dimensional gravity. Moreover, D-branes turn out to provide new contexts and new intuition for older mechanisms (like inflation) deemed necessary in cosmology, and to indicate new approaches to the problems of dark matter and the nature of the initial cosmological singularity.

In the search for new string vacua, an exciting new possibility emerged, namely that a compactification scale [24]-[27] or the string scale maybe much lower than the four-dimensional Planck scale [28]-32] and in fact as low as a few TeV, without grossly conflicting with available experimental data. Such ground states, namely orientifolds, can be considered as generalized compactifications of type-I string theory. They contain D-branes whose (localized) fluctuations should describe the SM fields. This gives flesh and blood to earlier ideas of a brane-universe [33]- 35], gives a new perspective of the hierarchy problem [36], borrowing on ideas from the gaugetheory/gravity correspondence and provides new realizations of four-dimensional gravity [37]. Moreover, it provides novel contexts for the early-universe cosmology [38]-[63].

\section{A survey of various string theory compactifications}

Different string theories have distinct ways of realizing the gauge interactions that are responsible for the SM forces. Ten-dimensional gravity is always an ingredient, coming from the closed string sector. The simplest way to convert it to four dimensional gravity is via compactification and this is what we will assume here. In section 6 we will describe other ways of turning higher-dimensional gravity to four-dimensional, but the implementation of such ideas in string theory is still in its infancy.

Upon compactification to four dimensions on a six-dimensional manifold of volume $V_{6} M_{s}^{-6}$ the four-dimensional Planck scale is given at tree level by

$$
M_{P}^{2}=\frac{V_{6}}{g_{s}^{2}} M_{s}^{2}
$$

where $g_{s}$ is the string coupling constant and the volume $V_{6}$ is measured in string units. We have dropped numerical factors of order one.

\subsection{The heterotic string}

The ten-dimensional theory, apart from the gravitational super-multiplet, contains also a (super) Yang-Mills (sYM) sector with gauge group $E_{8} \times E_{8}$ or $S O(32)$.

Here, the gauge fields descent directly from ten dimensions, and we obtain for the four-dimensional gauge coupling constants

$$
\frac{1}{g_{Y M}^{2}}=\frac{V_{6}}{g_{s}^{2}}
$$


Such tree-level relations are corrected in perturbation theory and the couplings run with energy. The tree-level couplings correspond to their values at the string (unification) scale. In a stable and reliable perturbation theory, such corrections are small and this indicates that in order to comply with experimental data $g_{Y M} \sim \mathcal{O}(1)$ and (2.1,2.1.1) imply that

$$
M_{P}^{2}=\frac{M_{s}^{2}}{g_{Y M}^{2}} \quad \Rightarrow \quad M_{P} \sim M_{s}
$$

Thus, we obtain that for realistic perturbative vacua, the unification scale is tied to the four-dimensional Planck scale. This is a remarkable postdiction of the heterotic string that answers question B'.

The issue of supersymmetry breaking is of crucial importance in order to eventually make contact with the low-energy dynamics of the Standard Model.

There are two alternatives here, gaugino condensation (dynamical) and ScherkSchwarz (geometrical) supersymmetry breaking.

The first possibility can be implemented in the heterotic string, however it involves non-perturbative dynamics and consequently it is not well-controllable in perturbation theory. Moreover, we do not know how to describe this dynamics at the string level.

If supersymmetry is broken à la Scherk-Schwarz, then, the supersymmetry breaking scale is related to the size $R$ of an internal compact direction as

$$
M_{\text {susy }} \sim \frac{1}{R}
$$

The successful resolution of the hierarchy problem requires that $M_{\text {susy }} \sim$ a few $\mathrm{TeV}$ so that $M_{\text {susy }} / M_{P}<<1$. This implies, $R>>M_{s}^{-1}$ and from (2.1.1) $g_{s}>>1$ in order to keep $g_{Y M} \sim \mathcal{O}(1)$. Thus, we are pushed in the non-perturbative regime. To obtain information, on the strong coupling dynamics of the heterotic string we must use non-perturbative dualities. Under such dualities, the heterotic string is mapped to type-I string theory [18], type II theory [17] or heterotic M-theory [64]. A review of such dualities and their nontrivial tests can be found in [65, 66].

\subsection{The type-I string}

In ten dimensions, the strong coupling limit of the heterotic $\mathrm{SO}(32)$ string is the weakly-coupled type-I string. There are many non-trivial tests of this duality in ten or less dimensions [67]- [70].

In the Einstein frame, this duality implies

$$
G_{\mu \nu}^{I}=G_{\mu \nu}^{h e t} \quad, \quad A_{\mu}^{I}=A_{\mu}^{h e t} \quad, \quad g_{s}^{h e t}=\frac{1}{g_{s}^{I}} \quad, \quad M_{P}^{h e t}=M_{P}^{I}
$$


Since the ten-dimensional Planck scale, $\hat{M}_{P}$, is given by $\hat{M}_{P}^{8}=M_{s}^{8} / g_{s}^{2}$ in both theories we obtain from (2.2.1)

$$
M_{s}^{I}=\frac{M_{s}^{h e t}}{\sqrt{g_{s}^{h e t}}}
$$

Thus, at strong heterotic coupling, the dual type-I string scale is much smaller that the heterotic one: dual type-I strings have a much bigger size than the heterotic ones.

We are, however, interested in four-dimensional compactifications. It can be shown [71] that upon toroidal compactification to four-dimensions the strongly coupled heterotic string is dual to a weakly coupled type-I string when the compactification torus has 4,5 or 6 large dimensions.

In the type-I vacua, gauge symmetries can arise from $\mathrm{D}_{p}$-branes that stretch along the four Minkowski directions and wrap their extra $p-3$ dimensions in a submanifold of the compactification manifold. Let us denote by $V_{\|}$the volume of such a submanifold in string units.

The relation of the four-dimensional Planck scale to the string scale is the same as in (2.1) since gravity comes from the closed string sector. However, the fourdimensional YM coupling of the D-brane gauge fields now become

$$
\frac{1}{g_{Y M}^{2}}=\frac{V_{\|}}{g_{s}}
$$

and

$$
\frac{M_{P}^{2}}{M_{s}^{2}}=\frac{V_{6}}{g_{s} V_{\|}} .
$$

where $M_{P}$ is the four-dimensional Planck scale. Now $M_{s}$ can be much smaller than $M_{P}$ while keeping the theory perturbative, $g_{s}<1$, by having the volume of the space transverse to the $\mathrm{D}_{p}$ branes $\frac{V_{6}}{V_{\|}}>>1$. Thus, in this context, the string scale $M_{s}$ can be anywhere between the four-dimensional Planck scale and a few TeV without any obvious experimental objection [28]-[32]. The possibility of perturbative string model building with a very low string scale is intriguing and inherently interesting due to several reasons

- If $M_{s}$ is a few $\mathrm{TeV}$, string effects will be visible at experiments around that energy scale, namely accelerator experiments in the near future. If nature turns out to work that way, the experimental signals will be forthcoming. In the other extreme case $M_{s} \sim M_{P}$, there is little chance to see telltale signals of the string at $\mathrm{TeV}$-scale experiments.

- Supersymmetry can be broken directly at the string scale without the need for fancy supersymmetry breaking mechanisms (for example by direct orbifolding). Past the string scale there is no hierarchy problem since there is no field theoretic running of couplings. 
It seems that there is thus no-hierarchy problem to solve in this case. It is not difficult to realize, however, that here the essence of the problem is hidden elsewhere. Some of the internal dimensions in this context must be much larger than the string scale. The radii are expectation values of associated scalar fields that will generically have a potential in the absence of supersymmetry. The hierarchy problem now reads: why the minimum of the potential is at $R>>1$ ? Although we can imagine potentials with logs providing large hierarchies of their minima and computations in semi-realistic models indicate that this is possible [72, 73], it has not yet been achieved in realistic models in string theory.

\subsection{Type II string theory}

Another non-perturbative duality relates the heterotic string compactified on $T^{4}$ to type-IIA theory compactified on $K 3$ [17, 18]. There is by now direct [74] and indirect [75] non-trivial evidence for this.

This duality keeps the Einstein metrics and Planck scales of the two theories fixed, and inverts the six-dimensional string coupling $\tilde{g}_{s}$ defined as

$$
\frac{1}{\tilde{g}_{s}^{2}}=\frac{V_{4}}{g_{s}^{2}} \quad, \quad \tilde{g}_{s}^{h e t}=\frac{1}{\tilde{g}_{s}^{I I A}}
$$

Moreover, it dualizes the six-dimensional two-index antisymmetric tensor. In this way, the heterotic string is a magnetic string soliton of the electric type IIA string and vice versa.

It can be shown [71] that this duality maps a strongly coupled heterotic string to a weakly coupled type II string when we have 1,2,3,4,6 large dimensions.

In type II compactifications, the four-dimensional matter gauge fields come from the RR sector and generate abelian symmetries. There are no charged massive gauge fields in the perturbative spectrum to enhance the gauge symmetry to a nonabelian one. However, such enhancement can happen non-perturbatively. When two-cycles of the compactification manifold shrink to zero volume, states produced by $\mathrm{D}_{2}$ branes wrapping these cycles generate massless charged gauge bosons that enhance the symmetry to a non-abelian group.

We thus consider a compactification on $K_{3} \times T^{2}$ of volume $V_{K 3}$ and $V_{2}$ respectively in string units.

As in the heterotic case, the relation between the four-dimensional Planck scale and the string scale remains the same

$$
M_{P}^{2}=\frac{V_{K 3} V_{2}}{g_{s}^{2}} M_{s}^{2}
$$

On the other hand, since the non-abelian gauge symmetry comes from the RR sector there is no $V_{K 3}$ nor $g_{s}$ dependence in the four-dimensional YM coupling

$$
\frac{1}{g_{Y M}^{2}}=V_{2} \quad \Rightarrow \quad \frac{M_{P}^{2}}{M_{s}^{2}}=\frac{V_{K 3}}{g_{s}^{2}} \frac{1}{g_{Y M}^{2}}
$$


We can consequently generate a large hierarchy between $M_{P}$ and $M_{s}$ by choosing $V_{K 3}>>1$ or $g_{s}<<1$.

The second case with $V_{K 3} \sim 1$ and $g_{s} \sim 10^{-14}$ was explored in [76] and new interesting features of the dynamics uncovered. It is however, hard to construct calculable models since the physics at the singularity of the internal space is related to Little String Theory (LST) [77] which is not very well understood.

\section{Orientifold vacua, chirality and supersymmetry}

An efficient way of constructing type-I vacua is in terms of orientifolds [10-16], an upgrade to the notion of orbifold in closed string theory.

One starts from a type IIB (flat) vacuum described by a CFT invariant under world-sheet parity $\Omega$. One then orbifolds by a group of discrete symmetries that also includes $\Omega$. The structure of the orientifold group is then $G=G_{1}+\Omega G_{2}$ where $G_{1,2}$ are symmetry groups of the CFT. At generic values of the toroidal moduli, such symmetries are (left and/or right) translations and rotations preserving the lattice.

The one-loop amplitude implementing the $\Omega$ projection is interpreted as a Kleinbottle amplitude [78, 79] and has potential ultraviolet divergences (tadpoles).

Such tadpoles can be interpreted as sources in space-time introduced by the orientifold $(\Omega)$ projection. They couple to the massless IIB fields, in particular the metric (so they have energy or tension), the dilaton and the RR-forms (under which they are minimally coupled). Such sources are localized in sub-manifolds of spacetime, typically hyper-planes and are known as orientifold planes, $O_{p}$. They are BPSlike (|tension $|=|$ charge $\mid$ ) and there are four different kinds in each odd dimension characterized by positive vs negative tension and positive vs negative RR charge. The most common orientifold planes have negative tension, a fact that comes handy in the search of a flat vacuum.

Flatness and stability will be assured if D-branes are introduced in a way that guarantees the cancellation of tadpoles (energy and charge). They can be thought of as the twisted sector of the $\Omega$ projection.

Thus, an orientifold vacuum can be described as a collection of orientifold planes and D-branes in various configurations. RR-tadpole cancellation is necessary for consistency, since it is equivalent to the vanishing of gauge charge in a compact space. NS-tadpole cancellation is equivalent to the vanishing of forces in the Dbrane/O-plane vacuum configuration. A vacuum with un-canceled NS tadpoles is not in equilibrium and this shows up at (open) one-loop. In particular, there will be

UV divergences that signal this lack of equilibrium. From the closed string point of view this is an IR instability.

Orientifold planes, unlike D-branes do not have fluctuations. This is as well since in the case of negative tension such fluctuations would have negative kinetic terms. 
However, acting as sources, they do couple to the massless sector fields of the closed sector.

\subsection{D-brane mechanics}

$\mathrm{D}_{p}$-branes are defined as sub-manifolds of space-time where the closed strings can open up and attach. In flat space-time, such open strings have Neumann boundary conditions along the $p+1$ longitudinal directions of a $\mathrm{D}_{p}$-brane and Dirichlet for the rest.

The massless spectrum of fluctuations of a single D-brane, according to the Polchinski prescription [19], is that of a vibrating open superstring with its two endpoints forced to be attached to the brane. For the flat type II theory, this gives a vector $A_{\mu}\left(x^{\nu}\right), 9-p$ scalars $\Phi_{I}\left(x^{\nu}\right)$ and a 16-component fermion. This is the dimensional reduction of the $\mathrm{D}=10 \mathrm{~N}=1$ gauge multiplet to $p+1$ dimensions. The expectation values of the scalars $\Phi_{I}$ are the transverse coordinates of the position of the brane.

$\mathrm{N}$ distinct parallel branes are described by the $N^{2}$ possible strings stretched between them, generating the spectrum (and interactions) of a $\mathrm{U}(\mathrm{N})$ sYM theory. Moving the branes apart, the gauge symmetry is Higgsed to $U(1)^{N}$ in the generic case.

In general, for a string coordinate with Neumann boundary conditions on both endpoints (NN), the mode expansion contains a center of mass momentum, no winding and integral oscillator modes. There are zero modes in the Ramond sector.

For a string coordinate with Dirichlet boundary conditions on both endpoints (DD), the mode expansion contains a winding number, no momentum and integral oscillator modes. There are again zero modes in the R-sector.

Finally, for a string coordinate with Dirichlet boundary conditions on one endpoint and Neumann on the other, (DN), the mode expansion contains no winding or momentum and half-integral oscillator modes. There are fermionic zero modes in the NS-sector.

T-duality acts on D-branes by interchanging $\mathrm{N}$ and $\mathrm{D}$ boundary condition. Thus, a T-duality in a direction transverse to a $\mathrm{D}_{p}$ brane transforms it into a $\mathrm{D}_{p+1}$ brane while T-duality in a direction longitudinal to a $\mathrm{D}_{p}$ brane transforms it into a $\mathrm{D}_{p-1}$ brane. T-duality splits O-planes and fractionalizes their charge.

An open string with one endpoint attached on a stack of $\mathrm{N}$ coincident parallel D-branes has a spectrum that transforms as the fundamental (or anti-fundamental depending on the orientation) representation of the $\mathrm{U}(\mathrm{N})$ gauge symmetry.

One important property that is crucial for model building is the issue of the reduction of the supersymmetry of the world-volume gauge theory. This is a prerequisite in order to obtain chiral four-dimensional fermions, an crucial ingredient of the standard model. 
As we will expand below, there are two such methods available in the context of orientifolds: internal world-volume magnetic fields [80, 81, 82, 84, 85] and branes stuck at orbifold singularities [86, 87]. The second option is an extreme case of the generic situation of branes wrapping non-trivial cycles of the compactification manifold.

\subsection{D-branes at orbifold singularities}

We will describe here the example of $\mathrm{D}_{3}$ and $\mathrm{D}_{7}$ branes stuck at orbifold singularities following 87].

We thus consider $n \mathrm{D}_{3}$ branes transverse to a $Z_{N}$ singularity. Since the brane description is local in this case, all that matters is the local structure of the singularity and this is similar to $R^{6} / Z_{N}$. The $Z_{N}$ rotation acts on the $\mathrm{SO}(6) \mathrm{R}$-symmetry quantum numbers of the massless brane fields. The vectors $A_{\mu}$ transform in the singlet, the fermions in the spinor and the scalars in the vector.

Complexifying the scalars by pairs, the $Z_{N}$ rotation acts on them as

$$
R_{\theta}=\operatorname{diag}\left(e^{2 \pi i \frac{b_{1}}{N}}, e^{-2 \pi i \frac{b_{1}}{N}}, e^{2 \pi i \frac{b_{2}}{N}}, e^{-2 \pi i \frac{b_{2}}{N}}, e^{2 \pi i \frac{b_{3}}{N}}, e^{-2 \pi i \frac{b_{3}}{N}}\right)
$$

while on the spinor it acts as

$$
S_{\theta}=\operatorname{diag}\left(e^{2 \pi i \frac{a_{1}}{N}}, e^{2 \pi i \frac{a_{2}}{N}}, e^{2 \pi i \frac{a_{3}}{N}}, e^{2 \pi i \frac{a_{4}}{N}}\right)
$$

with

$a_{1}=\frac{b_{2}+b_{3}-b_{1}}{2} \quad, \quad a_{2}=\frac{b_{1}-b_{2}+b_{3}}{2} \quad, \quad a_{3}=\frac{b_{1}+b_{2}-b_{3}}{2} \quad, \quad a_{4}=-\frac{b_{1}+b_{2}+b_{3}}{2}$

Moreover we can parameterize the action of the rotation on the Chan-Paton (CP) indices without loss of generality using the matrices

$$
\gamma_{3, \theta}=\operatorname{diag}\left(\mathbf{1}_{n_{0}}, \theta \mathbf{1}_{n_{1}}, \cdots, \theta^{N-1} \mathbf{1}_{n_{N-1}}\right)
$$

where $\theta=e^{\frac{2 \pi i}{N}}, n=\sum_{i=0}^{N-1} n_{i}$ and $\mathbf{1}_{n}$ is the unit $n \times n$ matrix.

The orbifold action on the gauge boson state is

$$
A_{\mu} \sim \psi_{-\frac{1}{2}}^{\mu}|\lambda\rangle \rightarrow \psi_{-\frac{1}{2}}^{\mu}\left|\gamma_{3, \theta} \lambda \gamma_{3, \theta}^{-1}\right\rangle
$$

where the matrix $\lambda$ keeps track of the $\mathrm{CP}$ indices.

Thus, the invariant gauge bosons must satisfy $\lambda=\gamma_{3, \theta} \lambda \gamma_{3, \theta}^{-1}$. The solutions to this equation are $n_{i} \times n_{i}$ block diagonal matrices, and thus the invariant gauge bosons are in the adjoint of $\prod_{i=0}^{N-1} U\left(n_{i}\right)$.

The three complex scalars $\Phi_{k}$ obtained from the complexification of the six real scalars, transform as

$$
\Phi_{k} \sim \psi_{-\frac{1}{2}}^{k}|\lambda\rangle \rightarrow e^{-2 \pi i \frac{b_{k}}{N}} \psi_{-\frac{1}{2}}^{k}\left|\gamma_{3, \theta} \lambda \gamma_{3, \theta}^{-1}\right\rangle
$$


and the invariant scalars must satisfy $\lambda=e^{2 \pi i \frac{b_{k}}{N}} \quad \gamma_{3, \theta} \lambda \gamma_{3, \theta}^{-1}$. The solution to this equation gives scalars in the following representation of the gauge group

$$
\text { scalars } \quad \rightarrow \quad \sum_{k=1}^{3} \sum_{i=0}^{N-1}\left(n_{i}, \bar{n}_{i-b_{k}}\right)
$$

Finally the fermions are labelled as

$$
\psi_{a} \sim\left|\lambda ; s_{1}, s_{2}, s_{3}, s_{4}\right\rangle
$$

where $s_{i}= \pm \frac{1}{2}$, with $\sum_{i=1}^{4} s_{i}=$ odd (GSO projection). The states with $s_{4}=-\frac{1}{2}$ correspond to left, four-dimensional Weyl fermions while $s_{4}=\frac{1}{2}$ corresponds to right, four-dimensional Weyl fermions. The $s_{1,2,3}$ spinor quantum numbers are R-symmetry spinor quantum numbers. We can thus label the 8 on-shell fermion states as $\left|\lambda ; \alpha, s_{4}\right\rangle$ where $\alpha=1,2,3,4$ is the R-spinor quantum number. The fermions transform as

$$
\left|\lambda ; \alpha, s_{4}\right\rangle \rightarrow e^{2 \pi i \frac{a_{\alpha}}{N}}\left|\gamma_{3, \theta} \lambda \gamma_{3, \theta}^{-1} ; \alpha, s_{4}\right\rangle
$$

and the invariant fermions satisfy $\lambda=e^{2 \pi i \frac{a_{\alpha}}{N}} \gamma_{3, \theta} \lambda \gamma_{3, \theta}^{-1}$ The solution to this equation gives left moving fermions in the following representation of the gauge group

$$
\text { left Weyl fermions } \quad \rightarrow \quad \sum_{\alpha=1}^{4} \sum_{i=0}^{N-1}\left(n_{i}, \bar{n}_{i+a_{\alpha}}\right)
$$

a representation that is generically chiral.

When $\sum_{i=1}^{3} b_{i}=0$ so that $a_{4}=0$ we have an $\mathrm{N}=1$ supersymmetric configuration (the rotation $\in S U(3) \subset S O(6)$ ) and this is an $\mathrm{N}=1$ orbifold singularity. The $a_{4}$ fermions become the gaugini while the $a_{1,2,3}$ fermions are the $\mathrm{N}=1$ supersymmetric partners of the scalars.

To cancel the twisted tadpoles we must introduce also $\mathrm{m}_{7}$ branes (that we take to be transverse to the last complex coordinate, the third plane).

For the 77 strings the story is similar with a new CP matrix

$$
\gamma_{7, \theta}= \begin{cases}\operatorname{diag}\left(\mathbf{1}_{m_{0}}, \theta \mathbf{1}_{m_{1}}, \cdots, \theta^{N-1} \mathbf{1}_{m_{N-1}}\right), & b_{3} \text { even, } \\ \operatorname{diag}\left(\theta \mathbf{1}_{m_{0}}, \theta^{3} \mathbf{1}_{m_{1}}, \cdots, \theta^{2 N-1} \mathbf{1}_{m_{N-1}}\right), & b_{3} \text { odd }\end{cases}
$$

The extra fields that are localized on the $\mathrm{D}_{3}$ brane world-volume come from the 37 and 73 strings. For such strings, there are 4 ND directions which provide 4 zero modes in the NS sector (directions $4,5,6,7$ ) while from the NN and DD directions we have zero modes in the $\mathrm{R}$ sector ( directions $2,3,8,9$ )

The invariant (complex) scalars (NS sector) must satisfy

$$
\lambda_{37}=e^{-i \pi \frac{b_{1}+b_{2}}{N}} \gamma_{3, \theta} \lambda_{37} \gamma_{7, \theta}^{-1}
$$




$$
\lambda_{73}=e^{-i \pi \frac{b_{1}+b_{2}}{N}} \gamma_{7, \theta} \lambda_{73} \gamma_{3, \theta}^{-1}
$$

with spectrum

$$
\begin{aligned}
& \sum_{i=0}^{N-1}\left[\left(n_{i}, \bar{m}_{i-\frac{b_{1}+b_{2}}{2}}\right)+\left(m_{i}, \bar{n}_{i-\frac{b_{1}+b_{2}}{2}}\right)\right], \quad b_{3} \text { even, } \\
& \sum_{i=0}^{N-1}\left[\left(n_{i}, \bar{m}_{i-\frac{b_{1}+b_{2}+1}{2}}\right)+\left(m_{i}, \bar{n}_{i-\frac{b_{1}+b_{2}-1}{2}}\right)\right], \quad b_{3} \text { odd }
\end{aligned}
$$

The invariant fermions coming from the R-sector must satisfy

$$
\begin{aligned}
& \lambda_{37}=e^{i \pi \frac{b_{3}}{N}} \gamma_{3, \theta} \lambda_{37} \gamma_{7, \theta}^{-1} \\
& \lambda_{73}=e^{i \pi \frac{b_{3}}{N}} \gamma_{7, \theta} \lambda_{73} \gamma_{3, \theta}^{-1}
\end{aligned}
$$

with spectrum

$$
\begin{aligned}
& \sum_{i=0}^{N-1}\left[\left(n_{i}, \bar{m}_{i+\frac{b_{3}}{2}}\right)+\left(m_{i}, \bar{n}_{i+\frac{b_{3}}{2}}\right)\right], \quad b_{3} \text { even, } \\
& \sum_{i=0}^{N-1}\left[\left(n_{i}, \bar{m}_{i+\frac{b_{3}-1}{2}}\right)+\left(m_{i}, \bar{n}_{i+\frac{b_{3}+1}{2}}\right)\right], \quad b_{3} \text { odd }
\end{aligned}
$$

A similar analysis holds for $\mathrm{D}_{7}$ branes stretched transverse to the other two planes.

Finally, the twisted tadpole cancelation conditions in the sector labeled by $k=$ $1,2, \cdots, N-1$ are

$$
\operatorname{Tr}\left(\gamma_{3, \theta}^{k}\right) \prod_{i=1}^{3}\left[2 \sin \frac{\pi k b_{i}}{N}\right]+\operatorname{Tr}\left(\gamma_{7, \theta}^{k}\right) \sum_{i=1}^{3} 2 \sin \frac{\pi k b_{i}}{N}=0
$$

For viable models twisted tadpoles have to be canceled and there are many solutions to this.

Thus, such configurations provide a generically chiral spectrum of four-dimensional fermions.

\subsection{Internal magnetic fields and branes intersecting at angles}

It is well known that magnetic fields can generate chirality. The reason is that an (internal) magnetic field splits the masses of spinors according to their spin quantum numbers, and if appropriately adjusted, it can lead to chiral pieces of a non-chiral spinor remaining massless while the other parts picking up a mass. This mechanism can be implemented also in open string theory [80, 81]. What we will show here 
is that there is a T-dual version of this mechanism for chirality generation that has a pure geometrical interpretation, namely strings stretched between intersecting branes.

Consider an open string that has Neumann boundary conditions on a Euclidean 2-plane with coordinates $x^{1,2}$.

$$
\left.\partial_{\sigma} x^{1}\right|_{\sigma=0}=0 \quad,\left.\quad \partial_{\sigma} x^{2}\right|_{\sigma=0}=0 \quad,\left.\quad \partial_{\sigma} x^{1}\right|_{\sigma=\pi}=0 \quad,\left.\quad \partial_{\sigma} x^{2}\right|_{\sigma=\pi}=0
$$

We will turn on a constant magnetic field for the gauge field associated with the $\sigma=\pi$ end-point of the string. The world-sheet action is

$$
\begin{aligned}
S & =\frac{1}{2 \pi \alpha^{\prime}}\left[\int d \tau d \sigma \frac{1}{2}\left(\partial_{\tau} x^{i} \partial_{\tau} x^{i}+\partial_{\sigma} x^{i} \partial_{\sigma} x^{i}\right)+\left.\int d \tau A_{i} \partial_{\tau} x^{i}\right|_{\sigma=\pi}\right] \\
& =\frac{1}{4 \pi \alpha^{\prime}}\left[\int d \tau d \sigma\left(\partial_{\tau} x^{i} \partial_{\tau} x^{i}+\partial_{\sigma} x^{i} \partial_{\sigma} x^{i}\right)-\left.\int d \tau \partial_{j} A_{i} x^{i} \partial_{\tau} x^{j}\right|_{\sigma=\pi}\right]
\end{aligned}
$$

where in the second line we integrated the boundary term by parts and dropped a total $\tau$-derivative. For a constant magnetic field threading the plane, $F_{i j}=B \epsilon_{i j}$. Varying $x^{i}$ to get the equations of motion and integrating by parts we obtain

$$
\begin{gathered}
\delta S=-\frac{1}{2 \pi \alpha^{\prime}} \int d \tau d \sigma \delta x^{i}\left(\partial_{\tau}^{2}+\partial_{\sigma}^{2}\right) x^{i}+ \\
+\frac{1}{2 \pi \alpha^{\prime}} \int d \tau\left[-\left.\delta x^{i} \partial_{\sigma} x^{i}\right|_{\sigma=0}+\left.\delta x^{i}\left(\partial_{\sigma} x^{i}+B \epsilon_{i j} \partial_{\tau} x^{j}\right)\right|_{\sigma=\pi}\right]
\end{gathered}
$$

we thus obtain the bulk free equations $\left(\partial_{\tau}^{2}+\partial_{\sigma}^{2}\right) x^{i}=0$ along with the modified boundary conditions

$$
\begin{array}{cl}
\left.\partial_{\sigma} x^{1}\right|_{\sigma=0}=0 \quad,\left.\quad \partial_{\sigma} x^{2}\right|_{\sigma=0}=0 \\
\partial_{\sigma} x^{1}+\left.B \partial_{\tau} x^{2}\right|_{\sigma=\pi}=0 \quad, \quad \partial_{\sigma} x^{2}-\left.B \partial_{\tau} x^{1}\right|_{\sigma=\pi}=0
\end{array}
$$

We will now perform a T-duality in the coordinate $x^{2}$ which exchanges $\partial_{\sigma} x^{2} \leftrightarrow$ $\partial_{\tau} x^{2}$. The T-dual boundary conditions now read

$$
\begin{gathered}
\left.\partial_{\sigma} x^{1}\right|_{\sigma=0}=0 \quad,\left.\quad \partial_{\tau} x^{2}\right|_{\sigma=0}=0 \\
\partial_{\sigma} x^{1}+\left.B \partial_{\sigma} x^{2}\right|_{\sigma=\pi}=0 \quad, \quad \partial_{\tau} x^{2}-\left.B \partial_{\tau} x^{1}\right|_{\sigma=\pi}=0
\end{gathered}
$$

The boundary conditions at the $\sigma=0$ end-point describe a string attached to a D-brane localized along the line $x^{2}=0$. On the other hand, the boundary conditions at the $\sigma=\pi$ end-point describe a string attached to a D-brane rotated by an angle $\theta$ counter-clockwise, where $B=-\cot \theta$. Otherwise stated the second D-brane is localized along the line $\sin \theta x^{1}+\cos \theta x^{2}=0$. 
The net result is that an open string coupled to a constant magnetic field is T-dual to a string stretched between two D-branes intersecting at an angle $\theta=$ $-\operatorname{arccot}(B)$.

The effect of the boundary conditions (3.3.7) on the oscillator expansion of the string is to eliminate momentum and winding terms, and to shift the oscillators $a_{n} \rightarrow a_{n+\theta}$. Thus, the overall effect is the same as that of an orbifold of the plane by a angle of rotation $\theta$. Similar remarks apply to the world-sheet fermions.

\subsection{Intersecting $D_{6}$-branes}

To obtain chiral four-dimensional spinors, no internal two-torus should be left intact. Thus, the full picture involves $D_{6}$ branes that intersect at each of the three internal tori ${ }^{1}$ [82-85].

$D_{6}$-branes can be obtained by performing a T-duality along 3 directions of the standard type-I string. A T-duality along a direction changes the orientifold projection from $\Omega$ to $\Omega I$ where $I$ flips the sign of the T-dualized coordinate [78].

We are thus led to consider IIB on $T^{6}$ moded by $\Omega I_{3}$. Introducing coordinates $x_{i}^{1}, x_{i}^{2}, i=1,2,3$ for the three two-tori, $I_{3}$ flips the sign of the $x_{i}^{2}$ coordinates. Turningon magnetic fields $B_{i}$ in the type-I theory corresponds to this T-dual version to $D_{6}$-branes intersections at angles $\theta_{i}$ on each of the three internal two-tori.

The $D_{6}$-branes stretch in the $3+1$ Minkowski directions and wrap a one-cycle in each of the three internal tori. Introducing the standard $a_{i}, b_{i}$ cycles on each torus, a given $D_{6}$ brane wraps the homology 3-cycle

$$
\left[\Pi_{A}\right]=\prod_{i=1}^{3}\left(m_{i}\left[a_{i}\right]+n_{i}\left[b_{i}\right]\right)
$$

where the product is a wedge product.

Since $\Omega I_{3}$ reflects the $\mathrm{D}$-branes along the $x_{i}^{2}$ coordinates, it maps a given $\mathrm{D}$-brane A to its mirror image A' wrapped on the cycle

$$
\left[\Pi_{A^{\prime}}\right]=\prod_{i=1}^{3}\left(m_{i}\left[a_{i}\right]-n_{i}\left[b_{i}\right]\right)
$$

At each (oriented) intersection there is a chiral fermion (localized in four dimensions) which is left-moving (right-moving) if the intersection numbers is $1(-1)$. Thus the net number of chiral fermions coming from the string between a brane $\mathrm{A}$ and a brane $\mathrm{B}$ is equal to the total intersection number

$$
I_{A B}=\int_{T^{6}}\left[\Pi_{A}\right] \wedge\left[\Pi_{B}\right]=\prod_{i=1}^{3}\left(m_{A}^{i} n_{B}^{i}-n_{A}^{i} m_{B}^{i}\right)
$$

\footnotetext{
${ }^{1}$ We consider a $T^{6}$ with moduli such that it factorizes into a product, for simplicity.
} 
where we have used

$$
\int_{T^{2}}[a] \wedge[b]=1
$$

In the general case, a $D_{p}$ brane wrapping a p-dimensional sub-torus of $T^{N}$, and a $D_{N-p}$ brane wrapping a $(N-p)$-dimensional sub-torus of $T^{N}$ intersect at points in $T^{N}$. Choosing coordinates $X^{I}, I=1,2, \cdots, N$ for $T^{N}, x^{a}, a=1,2, \cdots, p$ for $D_{p}$ and $\xi^{i}, i=1,2, \cdots, N-p$ for $D_{N-p}$, all of them periodic up to integers, the wrapping numbers are defined as

$$
X^{I}=N_{a}^{I} x^{a} \quad, \quad X^{I}=M_{i}^{I} \xi^{i}, \quad N_{a}^{I}, M_{i}^{I} \in \mathbb{Z}
$$

Then, the intersection number is

$$
I_{p, N-p}=\epsilon_{I_{1} I_{2} \cdots I_{N}} \epsilon^{a_{1} a_{2} \cdots a_{p}} \epsilon^{i_{1} i_{2} \cdots i_{N-p}} N_{a_{1}}^{I_{1}} \cdots N_{a_{p}}^{I_{p}} M_{i_{1}}^{I_{p+1}} \cdots N_{i_{N-p}}^{I_{N}}
$$

Considering now stacks of $N_{A} D_{6}$-branes, from the AA strings we obtain gauge bosons in the adjoint of $U\left(N_{A}\right)$ as well as scalars that are generically massive (and maybe tachyonic) [82, 84].

From the $\mathrm{AB}+\mathrm{BA}$ strings we obtain $I_{A B}$ chiral fermions in the $\left(N_{A}, \bar{N}_{B}\right)$ of $U\left(N_{A}\right) \times U\left(N_{B}\right)$. From the AB'+B'A strings we obtain $I_{A B^{\prime}}$ chiral fermions in the $\left(N_{A}, N_{B}\right)$ of $U\left(N_{A}\right) \times U\left(N_{B}\right)$. Finally, from the AA'+A'A strings we obtain chiral fermions in the symmetric or antisymmetric representation of $U\left(N_{A}\right)$. Their number is model-dependent.

The tadpole cancellation conditions specify that the D-branes should be put on top of the orientifold planes (wrapping the cycle $\left[\Pi_{O}\right]$ ) namely

$$
\sum_{\text {all branes }} N_{A}\left[\Pi_{A}\right]+\left[\Pi_{O}\right]=0
$$

By arranging the multiplicities $N_{A}$ and the wrapping numbers $m_{A}^{i}, n_{A}^{i}$, models can be obtained with the standard model gauge group, and the correct spectrum [82, 83, 84, 88. -91].

It should be mentioned that such intersecting configurations of D-branes break supersymmetry completely for arbitrary angles. If however $\sum_{i=1}^{3} \theta_{i}=0$ in a given intersection, then $\mathrm{N}=1$ supersymmetry is preserved locally.

\subsection{Supersymmetry breaking}

Supersymmetry in string theory is broken by compactification (ie. background fields). For the flat (orbifold or orientifold) compactifications supersymmetry is typically broken by the orbifold/orientifold projection.

We distinguish two types of orbifold actions on toroidal compactifications.

- Non-free susy-breaking orbifolds. In this case the orbifold action has fixed points and the compactification manifold is singular. At the singularities (orbifold 
planes) the twisted fields are localized. The breaking of supersymmetry here is "explicit" in the sense that there are no massive gravitini with masses below the string scale. Another way of saying this is that the supersymmetry breaking scale is the string scale, $M_{\text {susy }} \sim M_{s}$.

- Freely-acting susy-breaking orbifolds. Here the orbifold action has no fixed points, and the compactification manifold is flat and smooth. The lightest gravitino has a mass proportional to an inverse internal radius and thus the supersymmetry breaking scale is $M_{\text {susy }} \sim 1 / R$. Such compactifications are essentially stringy [5]-[8] Scherk-Schwarz compactifications [4], (as shown in [8]). Moreover, the nature of the supersymmetry breaking is spontaneous, since (broken) supersymmetric Ward identities are satisfied [92]. In the limit $R \rightarrow \infty$ supersymmetry is restored in one higher dimension.

\subsubsection{Supersymmetry breaking in low-string scale orientifolds}

As it was mentioned earlier, supersymmetry must break in a realistic string groundstate. When the string scale is low (in the $\mathrm{TeV}$ region) the supersymmetry must break around that scale. In the context of orientifold ground states this may be accomplished at the string level by a combination of the following mechanisms 93.[99, 72, 137, 138,

- Non-freely acting supersymmetry-breaking orbifolds in the bulk, that generically induce breaking in the open sector.

- Freely-acting (SS) supersymmetry breaking orbifolds in the bulk that may also induce breaking in the open sector. The breaking could affect all modes in the open sector, or only the massive ones.

- Intersecting branes induce supersymmetry breaking in the open sector. This also is related as we have seen to non-trivial internal magnetic fields on the branes [80, 81].

- The presence of anti-branes induces supersymmetry breaking in the open sector.

It is obvious that at tree level, supersymmetry may break in the open sector and/or in the bulk. We may imagine more involved situations in which a tree level breaking in the closed sector may be communicated by loop effects to the open sector and vice versa. Although this is possible, calculability is reduced in such cases.

It is also possible that different sectors of open strings may be invariant under different supersymmetries, and finally supersymmetry will be broken in a given sector by radiative corrections 104. Calculability is generically impaired also in such cases. It should be remembered that in the open string case, unlike the closed string case, essentially nothing is known on two- and higher-loop diagrams.

Many different mechanisms and combinations of supersymmetry breaking patterns have been investigated in toy models [100]- [102]. 
Thus, it seems that at the simplest level, supersymmetry must break at tree level in the open sector, namely in the standard model part of the open sector (The open sector can in principle contain hidden matter, interacting to the SM fields via particles coming from stretched/massive strings).

A priori we can imagine that supersymmetry may be intact at tree level in the closed sector. We will however argue that for (NS) tadpole cancellation, supersymmetry should also be broken in the closed sector.

Imagine an $\mathrm{N}=1$ bulk compactification (orientifold) in the closed sector with an open sector that breaks supersymmetry completely. The (open) supersymmetry breaking can be interpreted as coming from $\mathrm{D}$ and $\mathrm{F}$ auxiliary field expectation values that always give a positive contribution to the effective potential. The only term that could cancel such contributions and make the vacuum energy (dilaton/graviton tadpole) vanish is the supergravity auxiliary field [105. Thus, supersymmetry should be also broken in the bulk.

It is interesting that this effective supergravity picture fits nicely with the function of orientifold planes in orientifolds. Orientifold planes do not only cancel RR charges but also energy (since they carry negative tension). They can be interpreted as localized expectation values of the (higher-dimensional) supergravity auxiliary fields.

This picture is vague in the context of $\mathrm{N}>1$ four-dimensional supersymmetry since the full off-shell structure of the supergravity and the associated auxiliary fields are not well understood. However, we expect that it can be made precise in the $\mathrm{N}=1$ case. It has been also argued [72, 773 that the breaking of bulk supersymmetry cannot be hard $\left(M_{S U S Y}=M_{s}\right)$ since this induces quadratic instabilities $\left(\sim M_{P}^{2}\right)$ in the theory when large dimensions are present. This can be seen as follows: The tendimensional one-loop vacuum energy in this case is $\sim M_{s}^{10}$ and the four-dimensional one

$$
\Lambda_{4} \sim M_{s}^{4} V_{6} \sim M_{s}^{2} M_{P}^{2}
$$

where we have used (2.1). This indicates a hierarchy problem when $M_{P}>>M_{s}$.

The cancellation of RR tadpoles in a open string ground state is necessary for the consistency of the theory [106]. The cancellation of the NS tadpoles, on the other hand is equivalent to the equilibrium condition of the brane configuration, or in general of the ground-state. When equilibrium is not present, one-loop amplitudes are infinite and calculability is lost.

Since, in constructing a stringy SM, one-loop corrections are crucial for comparisons with data, such a situation seems doomed. Although we may consider shifting the background data to cancel the tadpoles perturbatively, this does not seem to be a calculationally promising avenue. It is thus imperative to cancel NS tadpoles at tree-level. 
A final issue is that of stability of the ground-state alias the absence of tachyons. We should consider separately the open and closed tachyons.

- Closed string tachyons signal an instability in the closed sector and must be canceled.

- Open string tachyons signal an instability of the brane configuration but that is what the SM wants! The standard Higgs (as well as other possible Higgses) are tachyons in the symmetric $S U(3) \times S U(2) \times U(1)$ vacuum. One may thus allow the appropriate tachyons at tree level, and this is the case in some semi-realistic models [137, 138, 82, 83, 84, 88]-[91]. Then the rolling of the Higgs to the minimum of its potential can be interpreted as a rearrangement of the brane configuration (brane recombination [107]). The flip side of this is that in the final ground state the branes do not seem flat anymore and further progress is needed in order to be able to calculate.

The other possibility 108, 109], which may be easily realized in orientifolds is that the candidate Higgs field is massless at tree level (and typically has a quartic term in its potential. At one-loop radiative corrections to its mass can be negative overall. In particular this is the case in the non-supersymmetric SM since fermions give negative contributions, and massive fermions dominate. Thus, one-loop correction to the Higgs mass is dominated by the top and is negative. The flip side of this situation is that two-loop calculations are beyond reach.

Finally we should mention that one may construct models with a string scale close to the Planck scale and unbroken supersymmetry [152-158.

\section{Anomalous $U(1) \mathrm{s}$ in six and four dimensions and their role in string model building}

In string theory, we often encounter $\mathrm{U}(1)$ gauge symmetries with an anomalous spectrum, namely $\operatorname{Tr}[Q] \neq 0, \operatorname{Tr}\left[Q^{3}\right] \neq 0$ etc. The theory is however free of the associated anomalies due to the presence of the generalized Green-Schwarz mechanism. The anomalous variation of the action is canceled by non-invariant, classical contributions of antisymmetric tensor fields. This mechanism works in various dimensions, with a prototypical example, the cancellation of mixed and reducible anomalies in the ten-dimensional heterotic and type-I string theories.

In four dimensions, anomalous U(1)'s have their anomalies canceled via their Stuckelberg coupling to a pseudoscalar axion. As a result, the gauge symmetry is broken and the anomalous U(1) gauge boson obtains a mass.

There are two reasons to be interested in this issue: First, it seems that (several) anomalous U(1) symmetries are unavoidable [108] in brane constructions of the SM. Second, the fate of the associated global symmetries (that may include baryon and lepton number) is of paramount phenomenological importance in such realizations 
of the SM. Finally, anomalous U(1) gauge bosons mix at tree level with the massive standard model gauge bosons and thus there are constraints coming from the SM $\rho$-parameter [113]. Also, the anomalous U(1) gauge bosons mediate at loop level several SM processes, like $g-2$ and data provide constraints on their masses [114.

A recent review on anomalies and anomalous $\mathrm{U}(1) \mathrm{s}$ in field theory with extra dimensions can be found in 103

\subsection{Anomalies and symmetries in four dimensions}

We will now consider an anomalous $\mathrm{U}(1)$ gauge field $A_{\mu}$ in a four-dimensional theory. Due to non-vanishing triangle diagrams

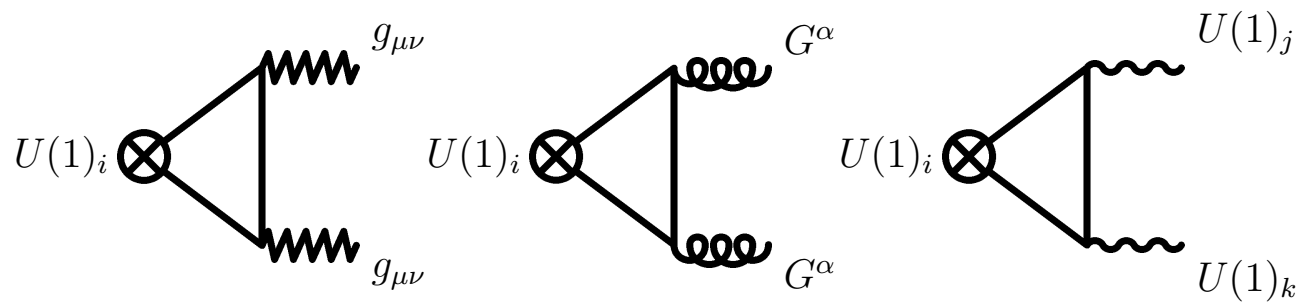

the effective action has an anomalous variation under a gauge transformation $A_{\mu} \rightarrow A_{\mu}+\partial_{\mu} \epsilon$

$$
\delta S_{\text {triangle }}=C \int d^{4} x \in\left(\operatorname{Tr}[Q] R \wedge R+\frac{1}{3} \operatorname{Tr}\left[Q^{3}\right] F \wedge F+\operatorname{Tr}\left[Q T^{a} T^{a}\right] \operatorname{Tr}[G \wedge G]\right)
$$

where $R$ is the gravitational two-form, $G$ the field strength of a non-abelian gauge boson and $C$ is a universal coefficient.

This one-loop anomalous variation is cancelled by an axion field $a$ that couples appropriately to the action:

$$
\begin{gathered}
S_{e f f}=\int d^{4} x\left[-\frac{1}{4 g^{2}} F_{\mu \nu} F^{\mu \nu}-\frac{M^{2}}{2}\left(\partial_{\mu} a+A_{\mu}\right)^{2}+\right. \\
\left.+C a\left(\operatorname{Tr}[Q] R \wedge R+\frac{1}{3} \operatorname{Tr}\left[Q^{3}\right] F \wedge F+\operatorname{Tr}\left[Q T^{a} T^{a}\right] \operatorname{Tr}[G \wedge G]\right)\right]
\end{gathered}
$$

Under the $\mathrm{U}(1)$ gauge transformation the axion transforms as $a \rightarrow a-\epsilon$, and since $\delta S_{\text {eff }}=-\delta S_{\text {triangle }}$ the full effective action, including the one-loop determinants is invariant. By choosing the physical gauge $a=0$ we can see that the $\mathrm{U}(1)$ gauge boson has acquired an (unormalized) mass $M$. We will indicate how to calculate this mass further in these lectures.

In heterotic $\mathrm{N}=1$ vacua there is a unique anomalous $\mathrm{U}(1)$ and its anomalies are cancelled by the universal two-index anti-symmetric tensor. In the type-I vacua, we may have several anomalous $\mathrm{U}(1) \mathrm{s}$ and in four-dimensional compactifications their anomalies are cancelled by a combination of RR untwisted and twisted axions [115, 116]. 
There are however, further effects associated with the anomalous U(1)'s and they are best visible when $\mathrm{N}=1$ supersymmetry is present. In this case, the axion belongs to a chiral multiplet, and has a CP-even partner $s$. When the axion comes from the twisted RR-sector, its partner is a scalar from the twisted NS sector. Its non-zero expectation value blows-up the orbifold singularity.

First, supersymmetry implies that there is a CP-even partner to the anomalycancelling CP-odd coupling of the axion. Thus, the D-brane gauge couplings have a non-trivial tree level correction of the form

$$
\frac{1}{g^{2}}=\frac{V_{\|}}{g_{s}}+\sum_{i} \lambda_{i} s_{i}
$$

where the sum is on the various twisted scalar partners of the axions that couple to the given gauge field and $\lambda_{i}$ are computable coefficients [117].

As it was shown in [118], at the orbifold fixed point $\left\langle s_{i}\right\rangle=0$. Once the orbifold is blown up, the tree-level gauge coupling changes.

Second, there is a D-term potential of the form

$$
V \sim \frac{1}{g^{2}} D^{2}=\frac{1}{g^{2}}\left(s+\sum_{i} Q_{i}\left|\phi_{i}\right|^{2}\right)^{2}
$$

where $Q_{i}$ are the $\mathrm{U}(1)$ charges of the scalars $\phi_{i}$. The minima of this potential are qualitatively distinct depending on the expectation value of $s$.

- $\langle s\rangle \neq 0$. Then, at least one charged scalar acquires a non-zero expectation value in order to minimize the D-term and the global U(1) symmetry is broken. This is the case in the heterotic string where $\langle s\rangle=\left\langle e^{\phi}\right\rangle=g_{s}$, and for non-trivial (interacting) vacua, $\langle s\rangle \neq 0$.

- $\langle s\rangle=0$. Here, the D-term is generically minimized by vanishing expectation values of the charged scalars and thus, although the gauge $\mathrm{U}(1)$ symmetry is broken (and the gauge boson massive), the associated global symmetry remains intact in perturbation theory.

This possibility is of utmost importance in D-brane model building, when the string scale is low. Potential baryon and lepton number violating operators are suppressed only by $M_{s} \sim \mathrm{TeV}$ and would be catastrophic unless baryon number is a good symmetry. It can be arranged that baryon number is an anomalous $\mathrm{U}(1)$ gauge symmetry, and its associated scalar stays at the orbifold point so that the symmetry remains as a global symmetry, to protect against fast proton decay [119, 120].

Non-perturbative effects (instantons) break the global symmetry. For anomalous $\mathrm{U}(1)$ s there are generically mixed anomalies with non-abelian groups. This indicates that the associated non-abelian instantons violate the global symmetry.

We should distinguish two cases here.

- The non-abelian group is unbroken and the interaction is strong. This implies a strong breaking of the global symmetry. This is the case of the axial U(1) flavor symmetry in QCD. 
- The non-abelian group symmetry is broken and the interaction necessarily weak. In this case the breaking is small. An example of this is the breaking of baryon number by weak $\mathrm{SU}(2)$ instantons, a process estimated by 't Hooft to be highly suppressed [121].

An important issue for phenomenology is the value for the physical mass of the anomalous $\mathrm{U}(1)$ gauge bosons. The physical mass depends on the following effective theory data.

(i) The UV mass $M$ in (4.1.2). This can be calculated only by a string calculation and we will describe it in a following section.

(ii) The mass has an additive contribution from the Higgs effect if the SM Higgs is charged under the anomalous $\mathrm{U}(1)$ in question (and it always is charged under at least one anomalous $\mathrm{U}(1))$.

(iii) The mass depends multiplicatively on the gauge coupling of the anomalous $\mathrm{U}(1)$.

An important question is, how much of the above discussion applies in the absence of unbroken supersymmetry?

The qualitative structure of anomaly cancellation remains the same. In orientifolds at tree level the D-term potential is as before, but it is corrected (typically mildly) at one-loop, and this is welcome in order to generate a negative mass-square for the Higgs. The UV mass remains the same (one-loop) since it is tied to anomalies. However, the contributions from the Higgs effect and the gauge coupling change in general at one loop.

\subsection{Anomalies in six dimensions}

An analysis of four-dimensional mixed U(1) anomalies, which can be done at the level of the massless (effective field theory) spectrum, is not enough to determine which of them obtain masses, [122, 123].

The reason is analogous to the fact that in the standard model massive quarks and leptons contribute to anomalies. As shown by 't Hooft, any fermion that can potentially become massless at some ground state of the theory, contributes to anomalies. For example, although SM fermions are massive in the broken vacuum, they are massless in the symmetric vacuum, and thus contribute to anomalies.

In our case, possible vacua of the theory correspond also to expectation values of the moduli scalars becoming infinite and the theory decompactifies to a higherdimensional string theory. Typically there is a part of the original spectrum which decompactifies uniformly (some states remain localized in four dimensions). This higher-dimensional theory may have anomalies not present in the four-dimensional theory. They are induced by the KK-descendants of the fermions that although they are massive in four dimensions, they become massless in higher dimensions. It is thus possible, that a non-anomalous $\mathrm{U}(1)$ of the four-dimensional theory, becomes 
an anomalous higher dimensional $\mathrm{U}(1)$. Thus, it is important to investigate the effect of the presence of higher-dimensional anomalies.

The first non-trivial higher-dimensional case arises in six dimensions. Here the leading anomalous diagrams are quadrangles with one insertion of the divergence of the $\mathrm{U}(1)$ current and the three further insertions of gauge fields (gravitons,abelian and non-abelian gauge fields) in various combinations.

Moreover, the available form gauge fields to cancel the anomalies are (up to duality) pseudo-scalars (axions) and two-index antisymmetric tensors (of which typically there are plenty in six-dimensional vacua) [115, 125].

It is enough to consider mixed anomalies of a single anomalous $U(1)$ with a single non-abelian group for our purposes.

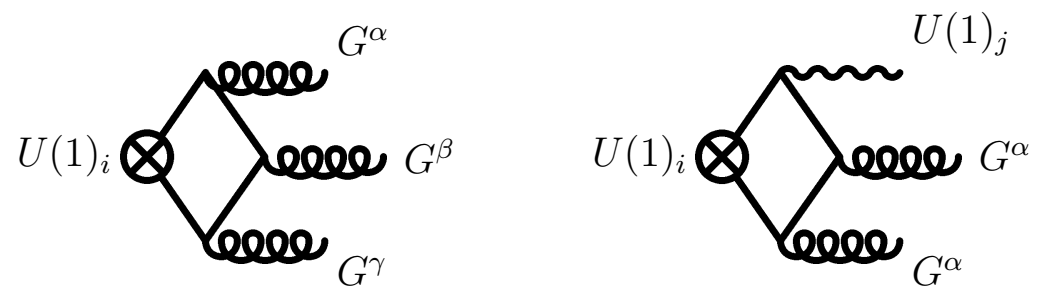

Gravitational and $\mathrm{U}(1)$ anomalies have a similar structure [115].

In six dimensions there are two distinct $\mathrm{U}(1) /$ non-abelian mixed anomalies.

- Those corresponding to $\operatorname{Tr}\left[Q T^{a}\left\{T^{b}, T^{c}\right\}\right]$, where $\mathrm{Q}$ is the $\mathrm{U}(1)$ charge generator and the diagrams involved have three insertions of gluons.

Such an anomaly induces a non-invariance of the effective action, under $\mathrm{U}(1)$ gauge transformations $A_{\mu} \rightarrow A_{\mu}+\partial_{\mu} \epsilon$,

$$
\left.\delta S_{\text {quadrangle }}=C \int d^{6} x \in \operatorname{Tr}\left[Q T^{a}\left\{T^{b}, T^{c}\right\}\right] G^{a} \wedge G^{b} \wedge G^{c}\right]
$$

where $G^{a}$ is the field strength of the non-abelian gauge boson.

This one-loop anomalous variation is cancelled by a pseudoscalar axion $a$ that couples as

$S_{e f f}=\int d^{6} x\left[-\frac{1}{4 g_{6}^{2}} F_{\mu \nu} F^{\mu \nu}-\frac{M_{6}^{2}}{2}\left(\partial_{\mu} a+A_{\mu}\right)^{2}+C a \operatorname{Tr}\left[Q T^{a}\left\{T^{b}, T^{c}\right\}\right] G^{a} \wedge G^{b} \wedge G^{c}\right]$

Under the $\mathrm{U}(1)$ gauge transformation the axion transforms as $a \rightarrow a-\epsilon$, and again since $\delta S_{\text {eff }}=-\delta S_{\text {quadrangle }}$ the full effective action, including the one-loop determinants is invariant.

In the presence of this type of six-dimensional anomaly, the anomalous $\mathrm{U}(1)$ gauge boson picks a six-dimensional unormalized mass $M_{6}$. With a gauge field scaling as mass and the axion being dimensionless, $g_{6}$ scales as length and $M_{6}$ as (mass) ${ }^{2}$. Upon compactification to four-dimensions on a two-dimensional compact manifold of volume $V_{2}$ the unormalized mass and the gauge coupling become

$$
\frac{1}{g_{4}^{2}}=\frac{V_{2}}{g_{6}^{2}} \quad, \quad M_{4}^{2}=V_{2} M_{6}^{2}
$$


and the normalized physical four-dimensional mass is

$$
M_{\mathrm{phys}}=g_{4} M_{4}=g_{6} M_{6}
$$

- Anomalies corresponding to $\operatorname{Tr}\left[Q^{2} T^{a} T^{a}\right]$, the diagrams involved having two insertions of gluons and one anomalous $\mathrm{U}(1)$ gauge boson. The one-loop non-invariance of the effective action, here is

$$
\delta S_{\text {quadrangle }}=C \int d^{6} x \in \operatorname{Tr}\left[Q^{2} T^{a} T^{a}\right] F \wedge G^{a} \wedge G^{a}
$$

and the anomaly is cancelled by a two-index antisymmetric tensor $B_{\mu \nu}$

$$
S_{\text {eff }}=\int d^{6} x\left[-\frac{1}{4 g_{6}^{2}} F_{\mu \nu} F^{\mu \nu}-\frac{1}{3 !} \hat{H}_{\mu \nu \rho} \hat{H}^{\mu \nu \rho}+\operatorname{Tr}\left[Q^{2} T^{a} T^{a}\right] B \wedge G^{a} \wedge G^{a}\right]
$$

where

$$
\hat{H}_{\mu \nu \rho}=\partial_{\mu} B_{\nu \rho}+2 A_{\mu} \partial_{\nu} A_{\rho}+\text { cyclic permutations } \sim d B+C S_{3}
$$

where $C S_{3}$ is the Chern-Simons 3 -form of the $\mathrm{U}(1)$ gauge field. Under the U(1) gauge transformation the antisymmetric tensor transforms as

$$
B_{\mu \nu} \rightarrow B_{\mu \nu}-\epsilon F_{\mu \nu}
$$

so that $\hat{H}_{\mu \nu \rho}$ is gauge invariant. The gauge variation is non-zero for the last term and since $\delta S_{\text {eff }}=-\delta S_{\text {quadrangle }}$ the full effective action, including the one-loop determinants is invariant.

Note that in this case, there is no mass generated for the anomalous $\mathrm{U}(1)$ gauge boson, and the associated $\mathrm{U}(1)$ gauge symmetry is not broken by the anomaly.

The general case involves a combination of the two types of mixed anomalies. A six-dimensional mass is generated only in the presence of the first type of anomaly.

Although there are no known eight-dimensional ground states with anomalous $\mathrm{U}(1) \mathrm{s}$ we will briefly describe the effects of anomalies in this case.

- A $\operatorname{Tr}\left[Q^{3} T^{a} T^{a}\right]$ anomaly is canceled by a four-form gauge field $C_{4}$ with a field strength $d C_{4}+F \wedge C S_{3}(A)$ and anomaly-canceling term $C_{4} \wedge G^{a} \wedge G^{a}$. The anomalous gauge boson remains massless.

- A $\left.\operatorname{Tr}\left[Q^{2} T^{a}\left\{T^{b}, T^{c}\right\}\right]\right]$ anomaly is canceled by a two-form gauge field $C_{2}$ with a field strength $d C_{2}+C S_{3}(A)$ and anomaly-canceling term $C_{2} \wedge G^{a} \wedge G^{b} \wedge G^{c}$. The anomalous gauge boson remains massless.

- A $\operatorname{Tr}\left[Q T^{4}\right]$ anomaly is canceled by an axion $a$ with kinetic term $(\partial a+A)^{2}$ and anomaly canceling term $a G^{a} \wedge G^{b} \wedge G^{c} \wedge G^{d}$. In this case the anomalous U(1) gauge boson acquires a mass. 


\subsection{String calculation of anomalous $\mathrm{U}(1)$ masses}

In view of the fact that anomalous $\mathrm{U}(1)$ symmetries are generic in D-brane realizations of the SM, their detailed study is of phenomenological importance for two reasons.

- Anomalous massive gauge bosons are a generic prediction and their masses can be at most as large as the string scale (or lower) which for low-scale vacua is in the $\mathrm{TeV}$ range. Moreover, it is possible that their UV masses be much smaller than the string scale [123] unlike the heterotic case. Thus, such gauge bosons can be produced in colliders, and they also affect, via their virtual effects, SM processes 114. Moreover, they generically mix with weak gauge bosons affecting their tree level couplings and may have measurable consequences [113. If their masses turn out to be much lower than the string scale, they may make such ground-states incompatible with existing experimental data.

- Four-dimensional U(1)'s free of four-dimensional anomalies, namely the hypercharge of the SM, may suffer in a given ground state of higher-dimensional anomalies that induce a mass term, rendering the model incompatible with experimental data.

It is thus obvious that a string calculation of their masses is important. Although we have argued that the presence or absence of mass can be detected by a careful study of anomalies in various decompactification limits, the precise value of the mass depends on the UV structure of the open (matter) sector and via UV/IR (open/closed) correspondence to IR data of the gravitational (closed) sector.

The mass can be indirectly calculated by a disk calculation of the mixing between the relevant axion and the anomalous $\mathrm{U}(1)$ gauge boson, or by a one-loop calculation of the two-gauge boson amplitude. Although the first method seems simpler, normalizing the amplitude is very difficult, except for the case of axions descending from the ten-dimensional RR forms (untwisted axions). Thus, generally the second calculation is preferable and we will sketch it below. The anomalous $U(1)$ masses were calculated in [123] for supersymmetric vacua and in [124] for non-supersymmetric vacua.

The two possible surfaces that can contribute to terms quadratic in the gauge boson at the one-loop level are the annulus and the Möbius strip. Of those, only the annulus with the gauge field vertex operators inserted at the two opposite ends has the appropriate structure to contribute to the mass-term. Indeed, vertex operators inserted at the same boundary will be proportional to $\operatorname{Tr}\left[\gamma_{k} \lambda^{a} \lambda^{b}\right]$, where $\gamma_{k}$ is the representation of the orbifold group element in the $k$-th orbifold sector acting on the Chan-Paton (CP) matrices $\lambda^{a}$. On the other hand, for gauge fields inserted on opposite boundaries, the amplitude will be proportional to $\operatorname{Tr}\left[\gamma_{k} \lambda^{a}\right] \operatorname{Tr}\left[\gamma_{k} \lambda^{b}\right]$ and it is this form of traces that determines the anomalous $U(1) \mathrm{s}$ [116]. As we show below, the mass comes from an UV contact term. The potential UV divergences that come from vertex operators inserted on the same boundary (both in cylinder and Möbius 
strip) cancel because of tadpole cancellation [126].

We must concentrate on the $C P$-even part of the amplitude which receives contributions only from even spin structures. This implies that we need the gauge boson vertex operators in the zero-ghost picture:

$$
V^{a}=\lambda^{a} \epsilon_{\mu}\left(\partial X^{\mu}+i(p \cdot \psi) \psi^{\mu}\right) e^{i p \cdot X},
$$

where $\lambda$ is the Chan-Paton matrix and $\epsilon^{\mu}$ is the polarization vector. Due to the structure of the vertex operators, the annulus amplitude for the two anomalous gauge bosons is $\mathcal{O}\left(p^{2}\right)$ before integration over the annulus modulus $t$. A contribution to the mass will be generated if a $1 / p^{2}$ pole appears after the $t$ integration. This can only come from the UV region $t \rightarrow \infty$. The IR region, only produces the standard $\log \left(p^{2}\right)$ running behavior of the effective gauge couplings. Thus, the UV tadpole responsible for the mass can be calculated directly in the closed string channel $(t \rightarrow 1 / t)$. For $\mathrm{N}=0,1$ sectors we obtain for the unormalized mass [123, 124]

$$
\left.M_{a b}^{2}\right|_{N=1}=\frac{1}{\pi^{3}} \sum_{N=1} \operatorname{Tr}\left[\gamma_{k} \lambda^{a}\right] \operatorname{Tr}\left[\gamma_{k} \lambda^{b}\right] \operatorname{Str} t_{k}\left[\frac{1}{12}-s^{2}\right]_{\text {massless closed channel }}
$$

where $s$ is the four-dimensional helicity. When the gauge fields come from different branes, then, the associated $\gamma$ matrices appear in the formula above.

The contribution of $\mathrm{N}=2$ sectors is volume-dependent. If the boundary conditions along the untwisted torus are $\mathrm{NN}$ and its volume (in string units) $V_{2}$, then

$$
\left.M_{a b}^{2}\right|_{N=2}=-\frac{2 V_{2}}{\pi^{3}} \sum_{N=2 \text { sectors }} \operatorname{Tr}\left[\gamma_{k} \lambda^{a}\right] \operatorname{Tr}\left[\gamma_{k} \lambda^{b}\right] \operatorname{Str} k\left[\frac{1}{12}-s^{2}\right]_{\text {massless open channel }}
$$

where here the helicity supertrace is the same in the open and closed channels. This in particular implies that volume dependent contributions to the anomalous $\mathrm{U}(1)$ masses can be calculated in the effective (open) field theory.

In the DD case, relevant for mass matrix elements coming from $D_{p<9}$ branes, the mass is similar as above with $\frac{V_{2}}{\alpha^{\prime}} \rightarrow \frac{\alpha^{\prime}}{V_{2}}\left(V_{2} \rightarrow 1 /\left(4 V_{2}\right)\right.$ for the $\alpha^{\prime}=1 / 2$ choice used here.

We can intuitively understand the volume-depended contributions of the anomalous $\mathrm{U}(1)$ masses from the following geometrical picture. The contribution to a given quadratic term $A_{\mu}^{i} A_{\mu}^{j}$ is mediated by all axions that mix at the disk level with both $A_{\mu}^{i}$ and $A_{\mu}^{j}$

Consider first the case of the two anomalous $\mathrm{U}(1)$ gauge bosons coming from the same stack of branes that stretch in the four-dimensional Minkowski space, $M_{4}$ and wrap an internal manifold $G_{A}$. The axion that couples to the U(1)'s lies on an orbifold plane stretching along $M_{4}$, and wrapping an internal manifold $G_{a}$. Let $G_{c}$ be the common submanifold of $G_{A}$ and $G_{a}$ with volume $V_{c}, V_{A}$ the volume of $G_{A}^{t}$ (the part of $G_{A}$ transverse to $G_{c}$ ) and $V_{a}$ the volume of $G_{a}^{t}$ (the part of $G_{a}$ transverse 
to $G_{c}$ ). Upon T-duality in the directions spanned by $G_{a}^{t}$ we obtain a D-brane that wraps the directions of $G_{a}$ but with volume $V_{c} / V_{a}$. The axion and the gauge fields now overlap over all directions along $G_{a}$.

Standard dimensional reduction now implies that

$$
M_{i j}^{2} \sim \frac{V_{c}}{V_{a}}
$$

and this captures the general behavior.

When the two gauge fields come from different stacks of branes, then (4.3.4) is still valid if in the previous discussion we substitute the D-brane world-volume with the common part of the two D-brane world-volumes.

The $\mathrm{N}=2$ contributions, linear in the internal volumes are effectively higherdimensional effects and thus sensitive to higher dimensional anomalies as advocated earlier.

\subsection{An example: anomalous $U(1)$ masses in the $Z_{6}^{\prime}$ orientifold}

The orbifold rotation vector is $\left(v_{1}, v_{2}, v_{3}\right)=(1,-3,2) / 6$. There is an order two twist $(k=3)$ and we must have one set of D5-branes. Tadpole cancellation then implies the existence of 32 D9-branes and 32 D5-branes that we put together at one of the fixed points of the $Z_{2}$ action (say the origin). More details on this orientifold can be found in [127. The gauge group has a factor of $U(4) \times U(4) \times U(8)$ coming from the D9-branes and an isomorphic factor coming from the D5-branes. The $N=1$ sectors correspond to $k=1,5$, while for $k=2,3,4$ we have $N=2$ sectors.

The potentially anomalous $U(1)$ s are the abelian factors of the gauge group. The four-dimensional anomalies of these $U(1) \mathrm{s}$ (and their cancellation mechanism) were computed in [116]. The mixed anomalies with the six non-abelian groups are given by the matrix ${ }^{2}$

$$
\left(\begin{array}{cccccc}
2 & 2 & 4 \sqrt{2} & -2 & 0 & -2 \sqrt{2} \\
-2 & -2 & -4 \sqrt{2} & 0 & 2 & 2 \sqrt{2} \\
0 & 0 & 0 & 2 & -2 & 0 \\
-2 & 0 & -2 \sqrt{2} & 2 & 2 & 4 \sqrt{2} \\
0 & 2 & 2 \sqrt{2} & -2 & -2 & -4 \sqrt{2} \\
2 & -2 & 0 & 0 & 0 & 0
\end{array}\right),
$$

where the columns label the $U(1)$ s while the rows label the non-abelian factors $S U(4)_{9}^{2} \times S U(8)_{9} \times S U(4)_{5}^{2} \times S U(8)_{5}$. The upper $3 \times 3$ part corresponds to the 99 sector and the lower one to the 55 sector. As can be seen by this matrix, the two linear combinations $\sqrt{2}\left(A_{1}+A_{2}\right)-A_{3}$ and $\sqrt{2}\left(\tilde{A}_{1}+\tilde{A}_{2}\right)-\tilde{A}_{3}$ are free of mixed four-dimensional non-abelian anomalies. It can also be shown that they are also free of mixed $U(1)$ anomalies.

\footnotetext{
${ }^{2}$ Here we use a different normalization for the $U(1)$ generators than in [116].
} 
The un-normalized mass matrix has eigenvalues and eigenvectors [123]:

$$
\begin{gathered}
m_{1}^{2}=6 V_{2} \quad, \quad-A_{1}+A_{2} \\
m_{2}^{2}=\frac{3}{2 V_{2}}, \quad-\tilde{A}_{1}+\tilde{A}_{2} \\
m_{3,4}^{2}=\frac{5 \sqrt{3}+48 V_{3} \pm \sqrt{3\left(25-128 \sqrt{3} V_{3}+768 V_{3}^{2}\right)}}{12}
\end{gathered}
$$

with respective eigenvectors

$$
\pm a_{ \pm}\left(A_{1}+A_{2}-\tilde{A}_{1}-\tilde{A}_{2}\right)-A_{3}+\tilde{A}_{3}
$$

where

$$
\begin{gathered}
a_{ \pm}=\frac{\mp 3+\sqrt{25-128 \sqrt{3} V_{3}+768 V_{3}^{2}}}{4 \sqrt{2}\left(4 \sqrt{3} V_{3}-1\right)} ; \\
m_{5,6}^{2}=\frac{15 \sqrt{3}+80 V_{3} \pm \sqrt{5\left(135-384 \sqrt{3} V_{3}+1280 V_{3}^{2}\right)}}{12},
\end{gathered}
$$

with respective eigenvectors

$$
\pm b_{ \pm}\left(A_{1}+A_{2}+\tilde{A}_{1}+\tilde{A}_{2}\right)+A_{3}+\tilde{A}_{3}
$$

where

$$
b_{ \pm}=\frac{ \pm 9 \sqrt{3}-\sqrt{5\left(135-384 \sqrt{3} V_{3}+1280 V_{3}^{2}\right)}}{4 \sqrt{2}\left(20 V_{3}-3 \sqrt{3}\right)}
$$

$V_{1,2,3}$ are the volumes of the three two-dimensional internal tori.

Note that the eigenvalues are always positive. They are also invariant under the T-duality symmetry of the theory $V_{2} \rightarrow 1 / 4 V_{2}$. Thus, all $U(1)$ s become massive, including the two anomaly-free combinations. The reason is that these combinations are anomalous in six dimensions [128]. Observe however, that in the limit $V_{3} \rightarrow 0$, the two linear combinations that are free of four-dimensional anomalies become massless. This is consistent with the fact that the six-dimensional anomalies responsible for their mass cancel locally in this limit [122, 123].

More examples can be found in 123, 124. The relationship between fourdimensional masses and six dimensional anomalies has been analyzed in detail in 128

\subsection{Physical axions}

When the Higgs effect is also at work in the presence of anomalous U(1)'s then there may remain physical massless axions in the spectrum. They are linear combinations

of the closed string axions responsible for anomaly cancellation and the Higgs phases. 
Such axions will obtain small masses from instanton effects. However, their couplings to matter are strongly constrained by experimental data, and it is thus important to understand to what extent they can be problematic. In D-brane realizations of the standard model we must have at least two Higgs fields in order to be able to give masses to all quarks and leptons [108]. Moreover, each Higgs is charged under hypercharge and an anomalous $\mathrm{U}(1)$.

We will analyze here the case of a single anomalous $U(1)$ coupled to a Higgs field in order to discuss the relevant effects.

Consider an anomalous $\mathrm{U}(1)$ gauge boson $A_{\mu}$ coupled to a complex Higgs $H$, an anomaly-canceling axion $a$, other (non)-abelian gauge fields $G$ and matter fermions $\psi$.

$$
\begin{aligned}
S=- & \frac{1}{4 g^{2}} F_{\mu \nu}^{2}-\frac{1}{2}\left(k \partial_{\mu} a+M A_{\mu}\right)^{2}+\mathcal{A}_{i} a \operatorname{Tr}\left[G_{i} \wedge G_{i}\right]- \\
& -\frac{1}{2}\left|\partial_{\mu} H+i e A_{\mu} H\right|^{2}+V(|H|)+\gamma H \psi \bar{\psi}
\end{aligned}
$$

where $\mathcal{A}_{i}$ is the mixed anomaly $\mathcal{A}_{i} \sim \operatorname{Tr}\left[Q T_{i}^{a} T_{i}^{a}\right]$. Parameterize $H=r e^{i \theta}$.

The relevant $\mathrm{U}(1)$ gauge transformations are

$$
A_{\mu} \rightarrow A_{\mu}+\partial_{\mu} \epsilon \quad, \quad a \rightarrow a-\frac{M}{k} \epsilon \quad, \quad \theta \rightarrow \theta-e \epsilon
$$

The minimum of the Higgs potential imposes $r=v$ and expanding around it (ignoring the radial fluctuations) the relevant action becomes

$$
S=-\frac{1}{4 g^{2}} F_{\mu \nu}^{2}-\frac{1}{2}\left(k \partial_{\mu} a+M A_{\mu}\right)^{2}+\mathcal{A}_{i} a \operatorname{Tr}\left[G_{i} \wedge G_{i}\right]-\frac{v^{2}}{2}\left|\partial_{\mu} \theta+e A_{\mu}\right|^{2}+\gamma v e^{i \theta} \psi \bar{\psi}
$$

We now redefine the scalars

$$
\begin{gathered}
\phi=k M a+e v^{2} \theta, \quad \chi=-e k^{2} a+k M \theta \\
a=\frac{k M \phi-e v^{2} \chi}{k^{2}\left(M^{2}+e^{2} v^{2}\right)} \quad, \quad \theta=\frac{e k \phi+M \chi}{k\left(M^{2}+e^{2} v^{2}\right)}
\end{gathered}
$$

The scalar $\chi$ is gauge invariant while $\phi$ transforms under gauge transformations.

The action now becomes

$$
\begin{gathered}
S=-\frac{1}{4 g^{2}} F_{\mu \nu}^{2}-\frac{1}{2} \frac{\left(\partial_{\mu} \phi+\left(M^{2}+e^{2} v^{2}\right) A_{\mu}\right)^{2}}{M^{2}+e^{2} v^{2}}-\frac{v^{2}}{2 k^{2}\left(M^{2}+e^{2} v^{2}\right)}\left(\partial_{\mu} \chi\right)^{2}+ \\
+\frac{\mathcal{A}_{i}\left(k M \phi-e v^{2} \chi\right)}{k^{2}\left(M^{2}+e^{2} v^{2}\right)} \operatorname{Tr}\left[G_{i} \wedge G_{i}\right]+\gamma v e^{\frac{i(e k \phi+M \chi)}{k\left(M^{2}+e^{2} v^{2}\right)}} \psi \bar{\psi}
\end{gathered}
$$

We will gauge fix $\phi=0$ (physical gauge) and rescale $\chi$

$$
\hat{\chi}=\frac{v}{k \sqrt{M^{2}+e^{2} v^{2}}} \chi
$$


to finally obtain

$$
\begin{gathered}
S=-\frac{1}{4 g^{2}} F_{\mu \nu}^{2}-\frac{M^{2}+e^{2} v^{2}}{2} A_{\mu}^{2}-\frac{1}{2}\left(\partial_{\mu} \hat{\chi}\right)^{2}- \\
-\frac{\mathcal{A}_{i} e v}{k \sqrt{M^{2}+e^{2} v^{2}}} \hat{\chi} \operatorname{Tr}\left[G_{i} \wedge G_{i}\right]+\gamma v e^{\frac{i M \hat{\chi}}{v \sqrt{M^{2}+e^{2} v^{2}}}} \psi \bar{\psi}
\end{gathered}
$$

The Yukawa coupling between the physical axion and the fermions is

$$
\gamma_{e f f}=\gamma \frac{M}{\sqrt{M^{2}+e^{2} v^{2}}}
$$

In order to suppress this interaction we must have $M<<e v$. Then

$$
S=-\frac{1}{4 g^{2}} F^{2}-\frac{e^{2} v^{2}}{2} A^{2}-\frac{1}{2}(\partial \hat{\chi})^{2}-\frac{\mathcal{A}_{i}}{k} \hat{\chi} \operatorname{Tr}\left[G_{i} \wedge G_{i}\right]+\gamma v e^{\frac{i M \hat{x}}{e v^{2}}} \psi \bar{\psi}
$$

Consider the anomalous gauge boson to come from a D-brane, and the axion from an orbifold plane. Call the internal volume of the common intersection $V_{c}$, while the totally transverse volumes $V_{A}$ and $V_{a}$.

Then

$$
\frac{1}{g^{2}}=\frac{V_{c} V_{A}}{g_{s}} \quad, \quad M^{2}=\frac{V_{c}}{V_{a}} M_{s}^{2} \quad, \quad k^{2}=\frac{V_{c} V_{a}}{g_{s}^{2}} \quad, \quad \mathcal{A}_{i} \sim \frac{a_{i}}{M_{s}}
$$

and

$$
M_{A}=\sqrt{g_{s}} \frac{e v}{\sqrt{V_{c} V_{A}}} \quad, \quad \gamma_{e f f}=\gamma \frac{M_{s}}{e v} \sqrt{\frac{V_{c}}{V_{a}}} \quad, \quad \mu_{i}=\frac{g_{s}}{\sqrt{V_{a} V_{c}}} \frac{a_{i}}{M_{s}}
$$

where $\mu_{i}$ is the axionic trilinear coupling to other gauge bosons. Doing a chiral transformation we can transfer the axionic couplings to the Yukawas:

$$
\hat{\gamma}_{e f f}=\frac{m_{\psi} M_{s}}{e v^{2}} \sqrt{\frac{V_{c}}{V_{a}}}+\frac{m_{\psi}}{M_{s}} \frac{g_{s}}{\sqrt{V_{a} V_{c}}}
$$

where we have used $m_{\psi}=\gamma v$. Thus, for suppression, we must have large $V_{a}$ and we can set $V_{c} \sim \mathcal{O}(1)$ since, as we will argue later, we want a minimum of large dimensions.

If $V_{A} \sim 1$, then the axion coupling is $\mathcal{O}(1)$ and the anomalous gauge-boson mass is of the order of the $Z^{0}$ mass. This is experimentally excluded. Thus $V_{A}$ should be large. Then, we end up with a gauge boson stretching in 4 large dimensions and we expect problems from supernovae energy-loss. To estimate it, let us recall the amplitude for KK-graviton energy loss from supernovae, 279

$$
P_{g} \sim \frac{1}{M_{P}^{2}}(R T)^{n} \sim g_{s}^{2} \frac{T^{n}}{M_{s}^{n+2}}
$$


where we assumed $\mathrm{n}$ large dimensions of common size $\mathrm{R}, \mathrm{T}$ is the temperature at the supernova core and the four-dimensional Planck scale is $M_{P}^{2}=R^{n} M_{s}^{n+2} / g_{s}^{2}$. The factor $R T$ counts the number of $\mathrm{KK}$ states that can be radiated away (per large dimension).

The similar rate for the emission of KK-states of a (massive) vector is

$$
P_{A} \sim g^{2}\left(R T-M_{A}\right)^{n_{A}} \frac{1}{T^{2}}
$$

where $g$ is the four-dimensional gauge coupling $g^{2}=g_{s} /\left(M_{s} R\right)^{n_{A}}$ and $n_{A}$ is the number of large dimensions felt by the gauge field. When $M_{A} \geq R T$ then the emission rate is suppressed kinematically. In the opposite case $M_{A}<<R T$ we have

$$
P_{A} \sim g_{s} \frac{T^{n_{A}-2}}{M_{s}^{n_{A}}}
$$

and

$$
\frac{P_{A}}{P_{g}} \sim \frac{1}{g_{s}}\left(\frac{M_{s}}{T}\right)^{n+2-n_{A}}
$$

Since $n-n_{A} \geq 0$, for low scale string models $\frac{P_{A}}{P_{g}} \geq 10^{8}-10^{10}$ and gauge boson emission provides more stringent constraints than KK-graviton emission.

These constraints can be avoided if $M_{A} \sim 30 \mathrm{MeV}$. This requires $V_{A} \sim 10^{7}$ with $\frac{g^{2}}{4 \pi} \sim 10^{-8}$. This may be allowed, however in this case there are again 4 large dimensions and there are only two string-size dimensions in which standard model branes can stretch.

A way out of this impasse is to have additional $\mathrm{U}(1)$ symmetry breaking effects in the potential, by moving, for example, away from the orbifold point.

\section{D-brane Standard Model building}

Our purpose here is to investigate closely the configuration of D-branes that can lead to the SM spectrum and gauge groups. In general, an orientifold ground state consists of a six-dimensional compactification manifold (with potential orbifold singularities). Embedded are $D_{p \geq 3}$ branes who stretch along Minkowski space and wrap the extra p-3 dimensions on appropriate cycles of the compact manifold. Also included are Orientifold planes that cancel the tadpoles of the theory.

Since masses of open strings are proportional to their lengths, it is obvious that the branes that give rise to the SM fields must be very close together in the internal space. Thus, we can talk about the local group of SM D-branes and we may focus our discussion on this. The presence of other branes further away may affect global rather than local properties of the model (but can be important for the overall stability of the configuration). 
For simplicity we will assume that the compact space is an orbifolded torus, but our discussion applies also to curved compactifications.

We will also focus on ground states with a string scale in the $\mathrm{TeV}$ range, because such ground states may have easily reachable experimental consequences for the future collider experiments.

The standard relation between the string scale and the Planck scale, namely $M_{P}^{2}=\frac{V_{6}}{g_{s}^{2}} M_{s}^{2}$ implies that the internal volume must be very large in string units. The hierarchy problem in this context is the question of what stabilises the value of $V_{6}>>1$. No compelling answer exists to this question so we will bypass it and move on.

Since $M_{s} \sim \mathrm{TeV}$, supersymmetry can break at the string scale.

An important constraint comes from the fact that a SM D-brane wrapping a large dimension automatically implies a multitude of KK states for the appropriate gauge bosons with small masses. These are experimentally unacceptable. Thus, D-branes generating the SM gauge group should wrap string-sized dimensions.

The interesting question is how many large dimensions the internal manifold can have. We have two competing effects. If there are several large dimensions, the space where we can stretch the SM branes is very much reduced, and it is difficult to generate the small differences of the SM gauge couplings (that in the simplest case are proportional to the internal volumes). Thus, it would seem that the optimum would correspond to one large dimension. However as shown in [129, in this case UV/IR duality implies power corrections to the effective field theory that destroy decoupling. The next best is two large dimensions, since in this case quantum corrections are soft (logarithmic) and this is what we will assume in the sequel.

There is another important ingredient in the SM and this is neutrinos with naturally light (e.g. $10^{-3}-1 \mathrm{eV}$ ) masses. The only known mechanism in open string theory that can achieve this, stipulates that right-handed neutrinos come from a brane wrapping one or more large dimensions [130.136.

The relevant effective action is

$$
S_{\text {neutrino }} \sim \int d^{4} x \quad\left(V \bar{\nu}_{R} \not \partial \nu_{R}+m \bar{\nu}_{R} \nu_{L}\right)
$$

where $V$ is large and $m \sim \mathcal{O}(1)$ since the brane carrying $\nu_{L}$ is intersecting the one of $\nu_{R}$ only along the four-dimensional Minkowski space. Normalizing the kinetic term to one, we obtain a mass $\sim m / \sqrt{V}$ which is naturally small. For this to be protected, large $\left(\sim M_{s}\right)$ Majorana masses for the neutrinos must be forbidden, and this is taken care of by good lepton number conservation.

Thus, in order to have naturally light neutrinos, at least one of the SM branes should wrap the large dimensions. There are several further questions that we will subsequently address. Concerning the structure of generations, as advocated earlier they may be obtained either from multiplicities in the case of branes at singularities or multiple intersections of branes. 


\subsection{Symmetries and Charges}

Extensions of the SM with a low string scale may have a priori severe problems with proton decay unless baryon number is a good global symmetry. It is similar with lepton number since it is an approximate symmetry of the low energy physics and is needed to protect small neutrino masses.

As we have argued earlier, in orientifolds, anomalous U(1) symmetries may remain as unbroken global symmetries in perturbation theory, broken only by nonperturbative effects. It is thus a requirement that the brane configuration guarantees that baryon and lepton number are such anomalous $\mathrm{U}(1)$ gauge symmetries.

We may now move on to discuss the embedding of the SM charges in the D-brane configuration.

The minimal $D$-brane configuration that can successfully accommodate the SM gauge group consists of three sets of branes with gauge symmetry $U(3)_{c} \times U(2)_{L} \times$ $U(1)_{1}$. The first set contains three coincident branes ("color" branes). An open string with one end attached to this set, transforms as an $S U(3)_{c}$ triplet (or antitriplet), but also carries an additional $U(1)_{c}$ quantum number which can be identified with the (gauged) baryon number. Similarly, $U(2)_{L}$ is realized by a set of two coincident branes ("weak" branes) and open strings attached to them from the one end are $S U(2)_{L}$ doublets characterized by an additional $U(1)_{L}$ quantum number, the (gauged) weak "doublet" number. Moreover, consistency of the SM embedding requires the presence of an additional $U(1)_{1}$ factor, generated by a single brane. This is needed for several reasons, the most important being mass generation for all quarks and leptons of the heaviest generation.

In all the above brane configurations there exist states (e.g. the $S U(2)_{L}$ singlet anti-quarks) which correspond to open strings with only one of their ends attached to one of the three sets of D-branes. The other end must go to another brane. This requires the existence of at least one more $\mathrm{U}(1)$ brane. Moreover, this brane can wrap a large dimension and can provide right-handed neutrinos with small masses.

Thus, we consider an additional D-brane (in the bulk) giving rise to an extra abelian gauge factor $U(1)_{b}$.

\begin{tabular}{|c|c|c|c|c|}
\hline particle & $U(1)_{c}$ & $U(1)_{L}$ & $U(1)_{1}$ & $U(1)_{b}$ \\
\hline$Q\left(\mathbf{3}, \mathbf{2}, \frac{1}{6}\right)$ & +1 & $w$ & 0 & 0 \\
$u^{c}\left(\overline{\mathbf{3}}, \mathbf{1},-\frac{2}{3}\right)$ & -1 & 0 & $a_{1}$ & $a_{2}$ \\
$d^{c}\left(\overline{\mathbf{3}}, \mathbf{1},+\frac{1}{3}\right)$ & -1 & 0 & $b_{1}$ & $b_{2}$ \\
$L\left(\mathbf{1}, \mathbf{2},-\frac{1}{2}\right)$ & 0 & +1 & $c_{1}$ & $c_{2}$ \\
$e^{c}(\mathbf{1}, \mathbf{1},+1)$ & 0 & $d_{L}$ & $d_{1}$ & $d_{2}$ \\
\hline
\end{tabular}

Table 1: SM particles with their generic charges under the abelian part of the gauge group $U(3)_{c} \times U(2)_{L} \times U(1)_{1} \times U(1)_{b}$. 
The total gauge group is

$$
\begin{aligned}
G & =U(3)_{c} \times U(2)_{L} \times U(1)_{1} \times U(1)_{b} \\
& =S U(3)_{c} \times U(1)_{c} \times S U(2)_{L} \times U(1)_{L} \times U(1)_{1} \times U(1)_{b}
\end{aligned}
$$

and contains four abelian factors. The assignment of the SM particles is partially fixed from its non-abelian structure. The quark doublet $Q$ corresponds to an open string with one end on the color and the other on the weak set of branes. The anti-quarks $u^{c}, d^{c}$ must have one of their ends attached to the color branes. The lepton doublet and possible Higgs doublets must have one end on the weak branes. However, there is a freedom related to the abelian structure, since the hypercharge can arise as a linear combination of all four abelian factors. In a generic model, the abelian charges can be expressed without loss of generality in terms of ten parameters displayed in Table 1 .

We should stress here that we assume the minimal SM spectrum without superpartners and other exotics.

In a convenient parameterization, normalizing the $U(N) \sim S U(N) \times U(1)$ generators as $\operatorname{Tr} T^{a} T^{b}=\delta^{a b} / 2$, and measuring the corresponding $U(1)$ charges with respect to the coupling $g / \sqrt{2 N}$, the ten parameters are integers: $a_{1,2}, b_{1,2}, c_{1,2}, d_{2}=0, \pm 1$, $d_{1}=0, \pm 1, \pm 2, d_{L}=0, \pm 2, w= \pm 1$ satisfying

$$
\sum_{i=1,2}\left|a_{i}\right|=\sum_{i=1,2}\left|b_{i}\right|=\sum_{i=1,2}\left|c_{i}\right|=1, \sum_{i=1,2, L}\left|d_{i}\right|=2
$$

The first three constraints in (5.1.2) correspond to the requirement that the $u^{c}$ and $d^{c}$ anti-quarks, as well as the lepton doublet, must come from open strings with one end attached to one of the abelian D-brane sets. The fourth constraint forces the positron $e^{c}$ open string to be stretched either between the two abelian branes, or to have both ends attached to the abelian $U(1)_{1}$ brane, or to the weak set of branes. In the latter case, it has $U(1)_{L}$ charge \pm 2 and is an $S U(2)_{L}$ singlet arising from the antisymmetric product of two doublets. The parameter $w$ in Table 1 refers to the $U(1)_{L}$ charges of the quark-doublets, that we can choose to be \pm 1 , since doublets are equivalent with anti-doublets. Note that a priori one might also consider the case in which one of the $u^{c}$ and $d^{c}$ anti-quarks arises as a string with both ends on the color branes $(\mathbf{3} \times \mathbf{3}=\overline{\mathbf{3}}+\mathbf{6})$, so that its $U(1)_{c}$ charges would be \pm 2 . This, however, would invalidate the identification of $U(1)_{c}$ with the baryon number and forbid the presence of quark mass terms, since one of the combinations $Q u^{c}$ and $Q d^{c}$ would not be neutral under $U(1)_{c}$.

The hypercharge can in general be a linear combination of all four abelian group factors. However, we must restrict ourselves to models in which the bulk $U(1)_{b}$ does not contribute to the hypercharge. Since the $U(1)_{b}$ gauge coupling is tiny (because the brane wraps a large volume) if it participates in the hypercharge, it will force the hypercharge gauge coupling to be tiny, contrary to the experimental results. 
Hence,

$$
Y=k_{3} Q_{c}+k_{2} Q_{L}+k_{1} Q_{1} .
$$

The correct assignments for SM particles are reproduced, provided

$$
\begin{aligned}
k_{3}+k_{2} w=\frac{1}{6} \quad, \quad-k_{3}+a_{1} k_{1}=-\frac{2}{3} \quad, \quad-k_{3}+b_{1} k_{1}=\frac{1}{3} \\
k_{2}+c_{1} k_{1}=-\frac{1}{2} \quad, \quad k_{2} d_{L}+d_{1} k_{1}=1 .
\end{aligned}
$$

Notice that the above equations imply that $k_{1} \neq 0$.

The next step, after assigning the correct hypercharge to the SM particles, is to check for the existence of candidate fermion mass terms. Here, we discuss only the question of masses for one generation (the heaviest) and we do not address the general problem of flavor. To lowest order, the mass terms are of the form $Q d^{c} H_{d}^{\dagger}, Q u^{c} H_{u}$ and $L e^{c} H_{e}^{\dagger}$ where $H_{d}, H_{u}, H_{e}$ are scalar Higgs doublets with appropriate charges. For the generic charge assignments of Table [1, the required Higgs charges are

$$
\begin{aligned}
& H_{u}=\left(\mathbf{1}, \mathbf{2}, 0,-w,-a_{1},-a_{2}\right) \\
& H_{d}=\left(\mathbf{1}, \mathbf{2}, 0,+w,+b_{1},+b_{2}\right) \\
& H_{e}=\left(\mathbf{1}, \mathbf{2}, 0,1+d_{L}, c_{1}+d_{1}, c_{2}+d_{2}\right) .
\end{aligned}
$$

Provided the constraints (5.1.2) are satisfied, both $H_{u}$ and $H_{d}$ have the right charges and correspond to strings stretched between the weak and one of the abelian branes. Thus, (5.1.2) guarantees the existence of tree-level quark masses. On the other hand, the existence of $H_{e}$ depends on the particular choice of parameters, e.g. for $c_{1}+d_{1}=2, H_{e}$ does not exist and a tree-level lepton mass term $\left(L e^{c} H^{\dagger}\right)$ is forbidden. The generic constraint that guarantees tree-level lepton masses is

$$
\sum_{i=1,2}\left|c_{i}+d_{i}\right|=\left|1+d_{L}\right|=1 .
$$

The hypercharge constraints (5.1.4) can be easily solved. They require $a_{1} \neq b_{1}$ and

$$
\begin{aligned}
& k_{3}=\frac{a_{1}+2 b_{1}}{3\left(b_{1}-a_{1}\right)} \quad, \quad k_{2}=-\frac{\left(a_{1}+b_{1}\right)}{2\left(b_{1}-a_{1}\right) w} \quad, \quad k_{1}=\frac{1}{b_{1}-a_{1}} \\
& c_{1}=-\frac{b_{1}-a_{1}}{2}+\frac{\left(a_{1}+b_{1}\right)}{2 w} \quad, \quad d_{1}=b_{1}-a_{1}+\frac{\left(a_{1}+b_{1}\right) d_{L}}{2 w} .
\end{aligned}
$$

Using charge conjugation, it is sufficient to search for solutions with $\left(a_{1}, b_{1}\right) \in$ $\{(-1,0),(-1,1),(0,1)\}$. Solving for these choices, we get three allowed hypercharge embeddings:

$$
\begin{aligned}
& \text { (i) } a_{1}=-1, b_{1}=1: Y=\frac{1}{6} Q_{c}+\frac{1}{2} Q_{1} \\
& \text { (ii) } a_{1}=-1, b_{1}=0: Y=-\frac{1}{3} Q_{c}+\frac{w}{2} Q_{L}+Q_{1} \\
& \text { (iii) } a_{1}=0, b_{1}=1: Y=\frac{2}{3} Q_{c}-\frac{w}{2} Q_{L}+Q_{1} .
\end{aligned}
$$


Case (i) leads to $c_{1}=-1, c_{2}=0, d_{1}=2, d_{1}=d_{L}=0$. This is a special solution where the $U(1)_{b}$ brane decouples from the model since no SM particles are attached to it. It satisfies (5.1.6) and thus leads to tree level lepton masses. The solution exists for both $w= \pm 1$, as the value of $w$ does not play an important role when $k_{2}=0$. In case (ii), we have $c_{1}=-(1+w) / 2, d_{L}=0, d_{1}=1$ or $c_{1}=(1+w) / 2, d_{L}=2 w, d_{1}=d_{2}=0$, while case (iii) leads to $c_{1}=(w-1) / 2, d_{L}=0, d_{1}=1$ or $c_{1}=(1+w) / 2, d_{L}=2 w, d_{1}=$ $d_{2}=0$.

Combining the above three cases with the constraints (5.1.2) and (5.1.6), we get nine distinct configurations with tree-level quark and lepton masses. Out of these only four have a lepton number symmetry. We display these models in table 2 [108, 109].

\begin{tabular}{|c|c|c|c|c|c|c|c|c|c|c|c|c|}
\hline & $a_{1}$ & $a_{2}$ & $b_{1}$ & $b_{2}$ & $c_{1}$ & $c_{2}$ & $d_{1}$ & $d_{2}$ & $d_{L}$ & $w$ & $Y$ & $n_{h}$ \\
\hline 1 & -1 & 0 & 0 & -1 & 0 & -1 & 1 & 1 & 0 & -1 & $-\frac{1}{3} Q_{c}-\frac{1}{2} Q_{L}+Q_{1}$ & 2 \\
2 & 0 & 1 & 1 & 0 & 0 & -1 & 1 & 1 & 0 & 1 & $\frac{2}{3} Q_{c}-\frac{1}{2} Q_{L}+Q_{1}$ & 2 \\
3 & -1 & 0 & 0 & -1 & 0 & -1 & 0 & 0 & -2 & -1 & $-\frac{1}{3} Q_{c}-\frac{1}{2} Q_{L}+Q_{1}$ & 2 \\
4 & 0 & 1 & 1 & 0 & 0 & -1 & 0 & 0 & -2 & 1 & $\frac{2}{3} Q_{c}-\frac{1}{2} Q_{L}+Q_{1}$ & 2 \\
\hline
\end{tabular}

Table 2: The four brane configurations consistent with baryon and lepton number conservation.

We should note that in order for lepton number to appear as a gauged symmetry, the presence of $U(1)_{b}$ is crucial.

\subsection{Gauge couplings}

We will now match the gauge couplings of the configurations described in the previous section with the measured ones in order to determine the string scale.

We will have to be a bit more explicit about the brane configuration. The branes we can use are either $D_{3} / D_{7}$, or $D_{5} / D_{9}$ branes. The two sets can be interchanged by duality. We will focus on the second set. Then the Standard model particles must be associated with $D_{5}$ branes except for the $U(1)_{b}$ brane that can be either $D_{5}$ or $D_{9}$. We will take it to be a $D_{5}$ without loss of generality. We split, for simplicity, the six internal dimensions $4,5, \cdots, 9$ into three two-tori. The third two-torus along 8,9 is the one that has large volume.

Without loss of generality we take the world-volume of the $\mathrm{U}(3)$ branes to stretch along $0,1,2,3,4,5$, and that of the $\mathrm{U}(2)$ branes along $0,1,2,3,6,7$. We need the internal volumes wrapped to be distinct, in order to create the difference between the weak and the strong coupling constant. The $U(1)_{b}$ stretches then along $0,1,2,3,8,9$ and wraps the two large dimensions. the final $\mathrm{U}(1)$ brane must wrap the string size dimensions. We have two distinct options:

- It is parallel to the $\mathrm{U}(3)$ branes. Then (2.2.3) indicates that it must have the same gauge coupling at the string scale: $g_{1}=g_{3}$. 


\begin{tabular}{|c|c|c|c|c|c|c|}
\hline & $\left|k_{3}\right|$ & $\left|k_{2}\right|$ & $\left|k_{1}\right|$ & $M_{U}(\mathrm{TeV})$ & $g_{2}\left(M_{U}\right) / g_{3}\left(M_{U}\right)$ & $g_{2}\left(M_{U}\right) g_{3}\left(M_{U}\right)$ \\
\hline \multirow{4}{*}{$g_{1}=g_{3}$} & $\frac{1}{6}$ & 0 & $\frac{1}{2}$ & $4.6 \times 10^{20}$ & 1.1 & 0.21 \\
\cline { 2 - 7 } & $\frac{1}{3}$ & $\frac{1}{2}$ & 1 & $2.4 \times 10^{3}$ & 0.76 & 0.48 \\
\cline { 2 - 7 } & $\frac{2}{3}$ & $\frac{1}{2}$ & 1 & 7.2 & 0.65 & 0.61 \\
\hline \multirow{3}{*}{$g_{1}=g_{2}$} & $\frac{1}{6}$ & 0 & $\frac{1}{2}$ & $1.5 \times 10^{22}$ & 1.1 & 0.26 \\
\cline { 2 - 7 } & $\frac{1}{3}$ & $\frac{1}{2}$ & 1 & 0.32 & 0.57 & 0.73 \\
\cline { 2 - 6 } & $\frac{2}{3}$ & $\frac{1}{2}$ & 1 & - & - & - \\
\hline
\end{tabular}

Table 3: The string scale $M_{s}=2.5 M_{U}$ and the two independent gauge couplings for the two possible brane configurations and the various hypercharge embeddings.

- It is parallel to the $\mathrm{U}(2)$ branes. Then $g_{1}=g_{2}$.

Thus the strategy is to relate the two independent parameters $g_{2}, g_{3}$ and the string scale $M_{s}$ to the measured values of the three SM couplings. This will determine $g_{2,3}$ and the string scale.

In our normalizations, the hypercharge coupling $g_{Y}$ at the string scale is expressed as

$$
\frac{1}{g_{Y}^{2}}=\frac{6 k_{3}^{2}}{g_{3}^{2}}+\frac{4 k_{2}^{2}}{g_{2}^{2}}+\frac{2 k_{1}^{2}}{g_{1}^{2}}
$$

The one loop coupling evolution is given by $\left(\alpha_{i}=g_{i}^{2} / 4 \pi\right)$,

$$
\frac{1}{\alpha_{i}\left(M_{s}\right)}=\frac{1}{\alpha_{i}\left(M_{Z}\right)}+\frac{b_{i}}{4 \pi} \ln \frac{\Delta^{I} M_{s}}{M_{Z}},
$$

where $b_{3}=-7, b_{2}=-10 / 3+n_{h} / 6, b_{Y}=20 / 3+n_{h} / 6$ and $n_{h}$ is the number of scalar Higgs doublets.

The results are presented in Table 3. In the above calculations we have assumed that the number of doublets $n_{h}$ is the minimum $n_{h}=2$ required by the model.

It is obvious that the models that have consistent (small) values of the string scale are the third and the fifth in Table 3. Such values for the string scale are indicative since they may be modified by non-trivial threshold corrections.

On the other hand the coupling of $U(1)_{b}$ can be calculated from (2.1,2.2.3) to be

$$
g_{b}^{2}=\frac{2 g_{s}}{\alpha_{2} \alpha_{3}} \frac{M_{s}^{2}}{M_{P}^{2}}
$$

giving $g_{b} \sim 10^{-16}-10^{-14}$ when $M_{s} \sim 1-10 \mathrm{TeV}$.

There is finally another possibility that is relevant only for D-brane models with a string scale close to the four-dimensional Planck scale [110]. For such models the $\mathrm{U}(1)_{b}$ brane does not wrap a large dimension. It can consequently participate in 
the hypercharge. Taking this into account there is one more acceptable hypercharge embedding (up to signs) given by

$$
Q_{Y}=\frac{1}{6} Q_{3}-\frac{1}{2}\left(Q_{1}+Q_{b}\right)
$$

The spectrum of a model in this class is

$$
\begin{aligned}
& Q(\mathbf{3}, \mathbf{2},+1,-1,0,0) \quad, \quad u^{c}(\overline{\mathbf{3}}, \mathbf{1},-1,0,1,0) \quad, \quad d^{c}(\overline{\mathbf{3}}, \mathbf{1},-1,0,-1,0) \\
& L(\mathbf{1}, \mathbf{2}, 0,+1,0,1) \quad, \quad e^{c}(\mathbf{1}, \mathbf{1}, 0,0,-1,-1) \quad, \quad \nu_{R}(\mathbf{1}, \mathbf{1}, 0,0,1,-1)
\end{aligned}
$$

An economic solution of solving the tadpole conditions is putting the $U(1)_{1}$ brane on top of the color branes indicating again a "petite unification": $g_{3}=g_{1}$. This also produces the appropriate intersection numbers needed for family replication. Asking for the hypercharge gauge boson to remain massless indicates a symmetric configuration with $g_{b}=g_{2}$. We are thus left with two independent gauge couplings at the string scale. Fitting them to gauge coupling one obtains a "consistent" string scale of $M_{s} \sim 2 \times 10^{16} \mathrm{GeV}$ [110].

\subsection{Viable D-brane configurations}

So far, we have classified all possible $U(3)_{c} \times U(2)_{L} \times U(1)_{1} \times U(1)_{b}$ brane models that can successfully accommodate the SM spectrum. The quantum numbers of each model as well as the hypercharge embedding are summarized in Table 2 . Furthermore, compatibility with type I string theory with string scale in the TeV region, requires the bulk to be two-dimensional of (sub)millimeter size, and leads to two possible configurations: Place the $U(1)_{1}$ brane on top of the weak $U(2)_{L}$ stack of branes or on top of the color $U(3)_{c}$ branes. These impose two different brane coupling relations at the string (unification) scale: $g_{1}=g_{2}$ or $g_{1}=g_{3}$, respectively. For every model, using the hypercharge embedding of Table 2, the one loop gauge coupling evolution and one of the above brane coupling conditions, we can determine the unification (string) scale that reproduces the weak angle at low energies. The results are summarized in Table 3 .

We will now describe in more detail the four viable brane configurations that we label A, A',B,B'.

Models $A$ and $A^{\prime}$

We consider here the models 1 and 3 of Table 2, hereafter referred as models $A$ and $A^{\prime}$ respectively. They are characterized by the common hypercharge embedding

$$
Y=-\frac{1}{3} Q_{c}-\frac{1}{2} Q_{L}+Q_{1}
$$

but they differ slightly in their spectra. The spectrum of model $A$ is

$$
\begin{aligned}
& Q(\mathbf{3}, \mathbf{2},+1,-1,0,0) \quad, \quad u^{c}(\overline{\mathbf{3}}, \mathbf{1},-1,0,-1,0) \quad, \quad d^{c}(\overline{\mathbf{3}}, \mathbf{1},-1,0,0,-1) \\
& L(\mathbf{1}, \mathbf{2}, 0,+1,0,-1) \quad, \quad e^{c}(\mathbf{1}, \mathbf{1}, 0,0,+1,+1) \quad, \quad \nu_{R}(\mathbf{1}, \mathbf{1}, 0,0,0, \pm 2) \\
& H_{u}(\mathbf{1}, \mathbf{2}, 0,+1,+1,0) \quad, \quad H_{d}(\mathbf{1}, \mathbf{2}, 0,-1,0,-1)
\end{aligned}
$$



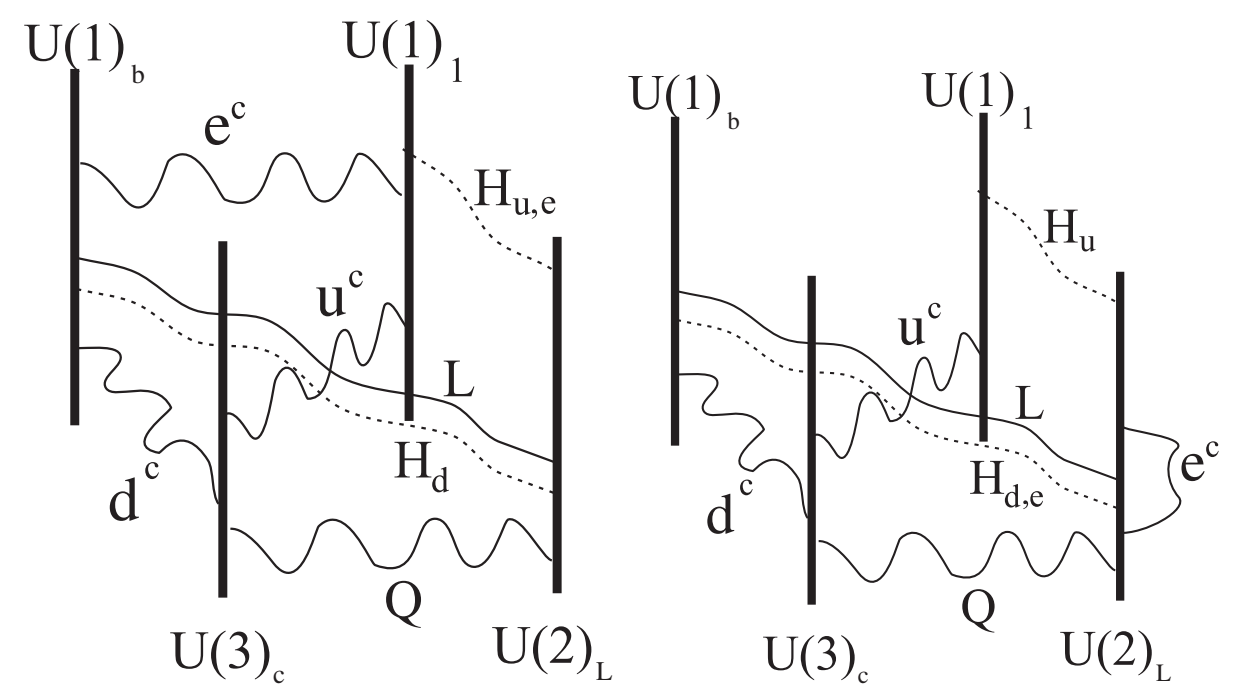

Figure 1: Pictorial representation of models $A, A^{\prime}$.

while in model $A^{\prime}$ the right-handed electron $e^{c}$ is replaced by an open string with both ends on the weak brane stack, and thus $e^{c}=(\mathbf{1}, \mathbf{1}, 0,-2,0,0)$. The two models are presented pictorially in Figure 1 .

Apart from the hypercharge combination (5.3.1) all remaining abelian factors are anomalous. Indeed, for every abelian generator $Q_{I}, I=(c, L, 1, b)$, we can calculate the mixed gauge anomaly $K_{I J} \equiv \operatorname{Tr} Q_{I} T_{J}^{2}$ with $J=S U(3), S U(2), Y$, and gravitational anomaly $K_{I 4} \equiv \operatorname{Tr} Q_{I}$ for both models $A$ and $A^{\prime}$ :

$$
K^{(A)}=\left(\begin{array}{cccc}
0 & -1 & -\frac{1}{2} & -\frac{1}{2} \\
\frac{3}{2} & -1 & 0 & -\frac{1}{2} \\
-\frac{3}{2} & \frac{1}{3} & -\frac{1}{3} & \frac{1}{6} \\
0 & -4 & -2 & -4
\end{array}\right), K^{\left(A^{\prime}\right)}=\left(\begin{array}{cccc}
0 & -1 & -\frac{1}{2} & -\frac{1}{2} \\
\frac{3}{2} & -1 & 0 & -\frac{1}{2} \\
-\frac{3}{2} & -\frac{5}{3} & -\frac{4}{3} & -\frac{5}{6} \\
0 & -6 & -3 & -5
\end{array}\right)
$$

It is easy to check that the matrices $K K^{T}$ for both models have only one zero eigenvalue corresponding to the hypercharge combination (5.3.1) and three non vanishing ones corresponding to the orthogonal $U(1)$ anomalous combinations. As discussed earlier, the three extra gauge bosons become massive, leaving behind the corresponding global symmetries unbroken in perturbation theory. The three extra $U(1)$ 's can be expressed in terms of known SM symmetries:

$$
\begin{aligned}
\text { Baryon number } B & =\frac{1}{3} Q_{c} \\
\text { Lepton number } \quad L & =\frac{1}{2}\left(Q_{c}+Q_{L}-Q_{1}-Q_{b}\right) \\
\text { Peccei-Quinn } \quad Q_{P Q} & =-\frac{1}{2}\left(Q_{c}-Q_{L}-3 Q_{1}-3 Q_{b}\right)
\end{aligned}
$$

Thus, our effective SM inherits baryon and lepton number as well as Peccei-Quinn (PQ) global symmetries from the anomaly cancellation mechanism. 
Accordind to our previous discussion of anomalous U(1) masses:

1. The two $U(1)$ combinations, orthogonal to the hypercharge and localized on the strong and weak D-brane sets, acquire in general masses of the order of the string scale from contributions of $N=1$ sectors, in agreement with effective field theory expectations based on $4 \mathrm{~d}$ anomalies.

2. Such contributions are not sufficient though to make heavy the third $U(1)$ propagating in the bulk, since the resulting mass terms are localized and suppressed by the volume of the bulk. In order to give string scale mass, one needs instead $N=2$ contributions associated to 6 d anomalies along the two large bulk directions.

3. Special care is needed to guarantee that the hypercharge remains massless despite the fact that it is anomaly free.

The presence of massive gauge bosons associated to anomalous abelian gauge symmetries is generic. Their mass is given by $M_{A}^{2} \sim g_{s} M_{s}^{2}$, up to a numerical model dependent factor and is somewhat smaller that the string scale. When the latter is low, they can affect low energy measurable data, such as $g-2$ for leptons 114 and the $\rho$-parameter [113], leading to additional bounds on the string scale.

An extension of the model is the introduction of a right-handed neutrino. A natural candidate state would be an open string ending on the $U(1)_{b}$ brane. Its charge is then fixed to +2 by the requirement of existence of the single possible neutrino mass term $L H_{d} \nu_{R}$. The suppression of the brane-bulk couplings due to the wave function of $\nu_{R}$ would thus provide a natural explanation for the smallness of neutrino masses.

Coming to the issue of gauge couplings, in this configuration we must take the $U(1)_{1}$ brane on top of the weak branes, leading to $g_{1}=g_{2}$. The required string scale is low $M_{s} \sim \mathcal{O}(500) \mathrm{GeV}$ (300-800 GeV, depending on the threshold corrections), and could account for the stability of the hierarchy.

\section{Models $B$ and $B^{\prime}$}

Another phenomenologically promising pair of configurations consists of solutions 4 and 9 of Table 2, named hereafter $B$ and $B^{\prime}$, which corresponds to the hypercharge embedding

$$
Y=\frac{2}{3} Q_{c}-\frac{1}{2} Q_{L}+Q_{1}
$$

The spectrum is

$$
\begin{aligned}
& Q(\mathbf{3}, \mathbf{2},+1,+1,0,0) \quad, \quad u^{c}(\overline{\mathbf{3}}, \mathbf{1},-1,0,0,1) \quad, \quad d^{c}(\overline{\mathbf{3}}, \mathbf{1},-1,0,1,0) \\
& L(\mathbf{1}, \mathbf{2}, 0,+1,0,-1) \quad, \quad e^{c}(\mathbf{1}, \mathbf{1}, 0,0,+1,+1) \quad, \quad \nu_{R}(\mathbf{1}, \mathbf{1}, 0,0,0, \pm 2) \\
& H_{u}(\mathbf{1}, \mathbf{2}, 0,-1,0,-1) \quad, \quad H_{d}(\mathbf{1}, \mathbf{2}, 0,+1,+1,0)
\end{aligned}
$$



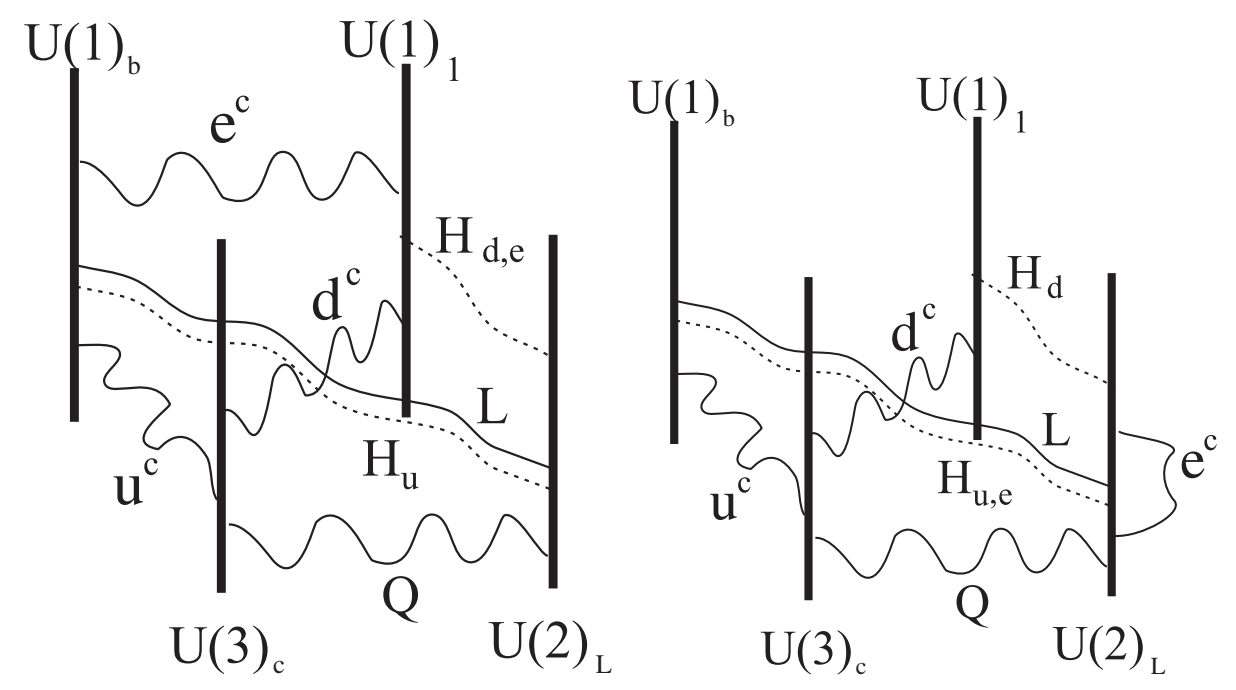

Figure 2: Pictorial representation of models $B$ and $B^{\prime}$.

for model $B$, while in $B^{\prime} e^{c}$ is replaced by $e^{c}(\mathbf{1}, \mathbf{1}, 0,-2,0,0)$. The two models are represented pictorially in Figure 2. The four abelian gauge factors are anomalous. Proceeding as in the analysis (5.3.2) of models $A$ and $A^{\prime}$, the mixed gauge and gravitational anomalies are

$$
K^{(B)}=\left(\begin{array}{cccc}
0 & 1 & \frac{1}{2} & \frac{1}{2} \\
\frac{3}{2} & 2 & 0 & -\frac{1}{2} \\
-\frac{3}{2} & \frac{2}{3} & \frac{4}{3} & \frac{11}{6} \\
0 & 8 & 4 & 2
\end{array}\right), K^{\left(B^{\prime}\right)}=\left(\begin{array}{cccc}
0 & 1 & \frac{1}{2} & \frac{1}{2} \\
\frac{3}{2} & 2 & 0 & -\frac{1}{2} \\
-\frac{3}{2} & -\frac{4}{3} & \frac{1}{3} & \frac{5}{6} \\
0 & 6 & 3 & 1
\end{array}\right)
$$

It is easy to see that the only anomaly free combination is the hypercharge (5.3.4) which survives at low energies. All other abelian gauge factors are anomalous and will be broken, leaving behind global symmetries. They can be expressed in terms of the usual SM global symmetries as the following $U(1)$ combinations:

$$
\begin{aligned}
& \text { Baryon number } B=\frac{1}{3} Q_{c} \\
& \text { Lepton number } \quad L=-\frac{1}{2}\left(Q_{c}-Q_{L}+Q_{1}+Q_{b}\right) \\
& \text { Peccei-Quinn } \quad Q_{P Q}=\frac{1}{2}\left(-Q_{c}+3 Q_{L}+Q_{1}+Q_{b}\right)
\end{aligned}
$$

The right handed neutrino can also be accommodated as an open string with both ends on the bulk abelian brane:

$$
\nu_{R}(\mathbf{1}, \mathbf{1}, 0,0,0,+2)+\nu_{R}^{c}(\mathbf{1}, \mathbf{1}, 0,0,0,-2)
$$

According to the RGE running results of Table 3, there is only one brane configuration, for the models under discussion, that reproduces the weak mixing angle 
at low energies. This consists of placing the $U(1)_{1}$ brane on the top of the color branes, so that $g_{1}=g_{3}$, which leads to $M_{s} \sim \mathcal{O}(10) \mathrm{TeV}$ (7-17 TeV, depending on the threshold corrections).

Finally we should stress that the triplication of families, can be obtained by the mechanisms of branes at singularities [87] or intersecting branes [82]- 84], 88, - 91].

\subsection{The quark and lepton mass structure}

An important ingredient in the realization of the SM is the structure of the quark and lepton masses and the associated Yukawa couplings. Although this problem remains open in the context of D-brane models, several features are evident at this level. The essential feature is that the Yukawa couplings relevant to fermion masses are constrained by the various $\mathrm{U}(1)$ symmetries and can present interesting patterns.

We will describe below the structure of the masses (and associated Yukawas) for the heaviest generation in the four low-scale string models of the previous section.

- Model A. The relevant Yukawa couplings compatible with all the gauge symmetries are

$$
M_{A}=\lambda_{u} Q u^{c} H_{u}+\lambda_{d} Q d^{c} H_{d}^{\dagger}+\lambda_{e} L e^{c} H_{u}^{\dagger}+\lambda_{\nu} L H_{d} \nu_{R}
$$

Here, charged leptons and up quarks (of the heaviest generation) obtain masses from the same Higgs $\left(H_{u}\right)$.

When all Yukawa couplings arise at the lowest (disk) order, it is easy to check that in the simplest case (absence of discrete selection rules, etc), they satisfy the following relations:

$$
\lambda_{u}=\lambda_{e}=\sqrt{2} g_{2}, \quad \lambda_{d}=\sqrt{2 g_{s}}, \quad \lambda_{\nu}=\sqrt{2} g_{b} .
$$

The central idea behind such relations is that couplings between two or three different branes scale with the common (intersection) volume.

Consequently the top and bottom quark masses are given by:

$$
m_{t}=g_{2} v \sin \beta \quad ; \quad m_{b}=\sqrt{g}_{s} v \cos \beta,
$$

where $\tan \beta=v_{u} / v_{d}$, with $v_{u}$ and $v_{d}$ the vacuum expectation values (VEVs) of the two higgses $H_{u}$ and $H_{d}$, respectively, and $v=\sqrt{v_{u}^{2}+v_{d}^{2}}=246 \mathrm{GeV}$. Note that in the case where the color branes are identified with D3 branes, one has $\sqrt{g_{s}}=g_{3}$, and in any case $g_{s} \geq g_{3}^{2}$. Note also that since the string scale in this model is relatively low, $M_{s} \lesssim 1 \mathrm{TeV}$, there is no much evolution of the low energy couplings from the electroweak to the string scale. Thus, using the known value of the bottom mass $m_{b} \simeq 4 \mathrm{GeV}$, one obtains for the top quark mass $m_{t} \simeq 162 \mathrm{GeV}$ which is less than $5 \%$ below its experimental value $m_{t}^{\exp }=174.3 \pm 5.1 \mathrm{GeV}$. In addition, the Higgs $\mathrm{VEV}$ ratio turns out to be large, $\tan \beta \simeq 100$. Note that such a large value is not 
in principle problematic as in the supersymmetric case, but it can lead to important higher order corrections.

On the other hand the $\tau$-mass is of the same order as the top mass, which is unrealistic. However, there is still the possibility that the lepton Yukawa coupling $\lambda_{e}$ vanishes to lowest order due to additional string discrete selection rules, and is generated by a higher dimensional operator of the form $L e^{c}\left(H_{u}^{\dagger} H^{\dagger} H\right)$ providing the appropriate suppression. ${ }^{3}$

- Model A'. The Yukawa couplings here are

$$
M_{A^{\prime}}=\lambda_{u} Q u^{c} H_{u}+\lambda_{d} Q d^{c} H_{d}^{\dagger}+\lambda_{e} L e^{c} H_{d}^{\dagger}+\lambda_{\nu} L H_{d} \nu_{R}
$$

with the same relation for the tree-level couplings as in (5.4.2). Using the parametrization in (5.4.3) we see that the relation of $m_{t}$ to $m_{b}$ is the same as in model A and the same remarks apply. Since here the lepton and down quark acquire their masses from the same Higgs, one obtains the phenomenologically interesting relation: $m_{b} / m_{\tau}=\sqrt{g_{s}} / g_{2}=g_{3} / g_{2}$, when strong interactions are on D3 branes. Thus, from Table 3, $m_{b} / m_{\tau} \simeq 1.75$ at the (string) unification scale, which is in the upper edge of the experimentally allowed region at the $Z$-mass, $1.46 \lesssim m_{b} /\left.m_{\tau}\right|_{\exp } \lesssim 1.75$. This relation could replace the successful GUT prediction $m_{b}=m_{\tau}$ of the conventional unification framework, in low scale string models. In conclusion model A' seems to be able to generate the required hierarchy of masses for the third generation.

- Model B. The relevant trilinear Yukawa couplings are,

$$
M_{B}=\lambda_{u} Q u^{c} H_{u}+\lambda_{d} Q d^{c} H_{d}^{\dagger}+\lambda_{e} L e^{c} H_{d}^{\dagger}+\lambda_{\nu} L H_{u} \nu_{R}
$$

The tree-level Yukawa couplings satisfy

$$
\lambda_{e}=\lambda_{u}=\sqrt{2 g_{s}}, \quad \lambda_{d}=\sqrt{2} g_{3}, \quad \lambda_{\nu}=\sqrt{2} g_{b}
$$

and we have

$$
m_{t}=\sqrt{g}_{s} v \sin \beta \quad ; \quad m_{b}=g_{3} v \cos \beta
$$

The first relation implies again a heavy top, while the bottom to tau mass ratio is now predicted, with a value $m_{b} / m_{\tau}=g_{3} / \sqrt{g_{s}} \lesssim 1$ which is apparently far from its experimental value. However, in this case, the string scale is relatively high and therefore one should take into account the renormalization group evolution above the weak scale. Solving the associated RGEs with the boundary conditions (5.4.7) and assuming $g_{3}=\sqrt{g_{s}}$, we obtain acceptable $m_{b}$ and $m_{\tau}$ masses for $M_{s} \sim 3 \times 10^{3}$ $\mathrm{TeV}$ and $\tan \beta \sim 80$. Note that the successful prediction of $m_{b}$ and $m_{\tau}$ is related to the condition $m_{b}=m_{\tau}$ at the (string) unification scale, which in the case of nonsupersymmetric Standard Model is obtained at relatively low energies [111, 112. 


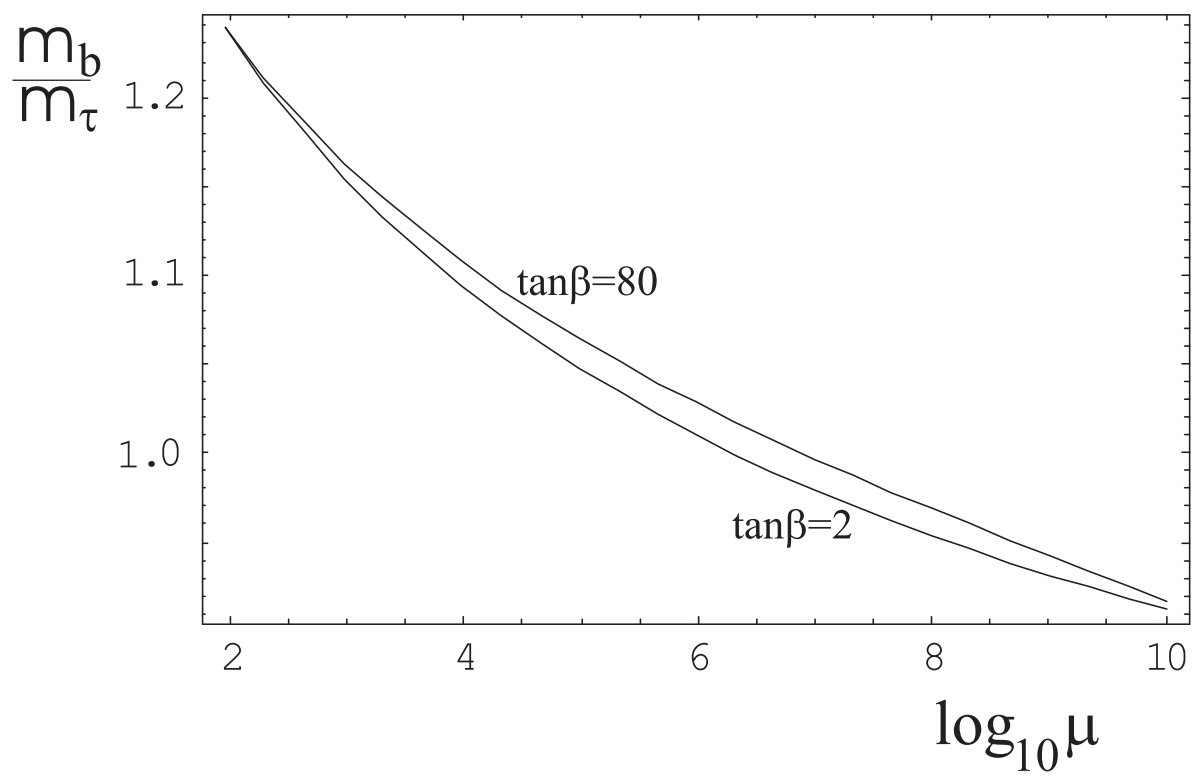

Figure 3: Evolution of the ratio $m_{b} / m_{\tau}$ as a function of the energy $\mu$ for $\tan \beta=2$ and $\tan \beta=80$. We have used as low energy parameters $m_{b}=4 \mathrm{GeV}, m_{\text {top }}=174 \mathrm{GeV}$ $a_{3}\left(M_{z}\right)=0.12, \sin ^{2} \theta_{W}=0.23113$.

Indeed, in Figure 3, we plot the mass ratio $m_{b} / m_{\tau}$ as a function of the energy, within the non supersymmetric Standard Model with two Higgs doublets. Nevertheless, the resulting value of $M_{s}$ is still significantly higher than the unification scale required from the analysis of gauge couplings in section 3. Moreover, the top quark mass turns out to be rather high, $m_{t} \sim 220 \mathrm{GeV}$. It is an open question wether this discrepancy can be attributed to threshold corrections that can be important in the case of two dimensional bulk 129.

- Model B'. The relevant Higgs couplings are given by

$$
M_{B^{\prime}}=\lambda_{u} Q u^{c} H_{u}+\lambda_{d} Q d^{c} H_{d}^{\dagger}+\lambda_{e} L e^{c} H_{u}^{\dagger}+\lambda_{\nu} L H_{u} \nu_{R}
$$

while the tree-level Yukawa couplings by

$$
\lambda_{u}=\sqrt{2 g_{s}}, \quad \lambda_{d}=\sqrt{2} g_{3}, \quad \lambda_{\nu}=\sqrt{2} g_{b} \text { and } \lambda_{e}=\sqrt{2} g_{2}
$$

Here, as in model A, the $\tau$ and top mass are of the same order and thus in conflict with experiment. As in model A, vanishing leading order coupling could be a way out.

In the discussion above we have paid attention to the heaviest generation. However, an important feature of the SM spectrum is the large hierarchy between the masses of the different generations. Can this be generated in the models above?

\footnotetext{
${ }^{3}$ Models with similar properties have been considered in the past in the perturbative heterotic string framework.
} 
A concrete idea in this direction si to generate such a hierarchy by exploiting the presence of world-sheet (disk) instanton corrections in models where branes intersect in the internal space [160. It relies on the fact that the BCFT three-point function responsible for the Yukawa couplings may have a prefactor coming from world-sheet instantons, if the relevant fields come from three branes that interest by-two in the internal space in such way as to create a triangle. Then the open string world-sheet must wrap this triangle and there is an exponential suppression due to the area of this triangle in space-time. Adjusting such areas it seem possible that an appropriate hierarchy of Yukawa couplings can be generated [160].

A further comment is due concerning neutrinos. By now there are stringent experimental limits in the mass matrix of neutrinos. A natural question is: how neutrinos with the right properties can be accommodated in D-brane models?

Right-handed neutrinos appear naturally in D-brane models. To generate (small) masses compatible with experiment, either one will have to wrap the associated brane around a large cycle, or produce the suppression from world-sheet instantons. Moreover, there are several possibilities in the neutrino sector.

A minimal possibility is to have a single right-handed neutrino which provides the mixing along with it KK-descendants. This possibility is rather constrained and was analyzed in [109]. Since in this case the KK-neutrinos are acting almost as sterile, there are stringent experimental constraints that indicate that one needs fine tuning in order that such a possibility to be compatible with data. On the other hand, if one has one right-handed neutrino per family then a priori there are are no such strong constraints. It remains however to be seen if there are brane models with a neutrino mass -matrix compatible with current data.

\begin{tabular}{|c||c|c|c|}
\hline$N_{i}$ & $\left(n_{i}^{1}, m_{i}^{1}\right)$ & $\left(n_{i}^{2}, m_{i}^{2}\right)$ & $\left(n_{i}^{3}, m_{i}^{3}\right)$ \\
\hline \hline$N_{3}=3$ & $(1,0)$ & $(2,1)$ & $(1,1 / 2)$ \\
\hline$N_{2}=2$ & $(0,-1)$ & $(1,0)$ & $(1,3 / 2)$ \\
\hline$N_{1}=1$ & $(1,3)$ & $(1,0)$ & $(0,1)$ \\
\hline$N_{1^{\prime}}=1$ & $(1,0)$ & $(0,-1)$ & $(1,3 / 2)$ \\
\hline
\end{tabular}

Table 4: Example of D6-brane wrapping numbers giving rise to the right number of SM families (from ([90, 91]). 


\subsection{The Standard Model structure from intersecting D-branes}

We will describe in this section, as an example a non-supersymmetric D-brane model utilizing intersecting D6-branes that gives the correct spectrum [90, 91].

We will be using the results and notation of section 3.4. The important ingredients are four stacks of branes that generate the minimal brane gauge group $U(3) \times U(2) \times U(1) \times U(1)^{\prime}$. We will label the associated stack of branes by the indices $3,2,1,1^{\prime}$. The hypercharge embedding is given by the possibility (5.2.4)

$$
Y=\frac{1}{6} Q_{3}-\frac{1}{2}\left(Q_{1}-Q_{1^{\prime}}\right)
$$

To generate the three families of the SM we will need some specific intersection numbers (defined in (3.4.3)) among the four stacks of branes

$$
\begin{aligned}
& I_{3,2}=1 ; I_{3,2 *}= 2 \\
& I_{3,1}=-3 ; I_{3,1 *}=-3 \\
& I_{2,1^{\prime}}=0 ; I_{2,1^{\prime} *}=-3 \\
& I_{1,1^{\prime}}=-3 ; I_{1,1^{\prime} *}=3
\end{aligned}
$$

These can be achieved by choosing the wrapping numbers on the three two-tori as in table 1 . A negative number denotes that the corresponding fermions should have opposite chirality to those with positive intersection number.

\begin{tabular}{|c|c|c|c|c|c|c|c|}
\hline Intersection & Matter fields & & $Q_{3}$ & $Q_{2}$ & $Q_{1}$ & $Q_{1}^{\prime}$ & $\mathrm{Y}$ \\
\hline \hline$(3,2)$ & $Q_{L}$ & $(3,2)$ & 1 & -1 & 0 & 0 & $1 / 6$ \\
\hline$\left(3,2^{*}\right)$ & $q_{L}$ & $2(3,2)$ & 1 & 1 & 0 & 0 & $1 / 6$ \\
\hline$(3,1)$ & $u_{R}$ & $3(\overline{3}, 1)$ & -1 & 0 & 1 & 0 & $-2 / 3$ \\
\hline$\left(3,1^{*}\right)$ & $d_{R}$ & $3(\overline{3}, 1)$ & -1 & 0 & -1 & 0 & $1 / 3$ \\
\hline$\left(2,1^{*}\right)$ & $L$ & $3(1,2)$ & 0 & -1 & 0 & -1 & $-1 / 2$ \\
\hline$\left(1,1^{\prime}\right)$ & $e_{R}$ & $3(1,1)$ & 0 & 0 & -1 & 1 & 1 \\
\hline$\left(1,1^{*}\right)$ & $\nu_{R}$ & $3(1,1)$ & 0 & 0 & 1 & 1 & 0 \\
\hline
\end{tabular}

Table 5: Standard model spectrum and $U(1)$ charges (from [90, 91])

The massless fermions living at the intersections are shown in table [. As we have argued in section 3.4, due to the orientifold operation one has to include the D6-branes which are "mirror" under that operation and have the same wrapping numbers except for a flip in sign for the $m_{a}^{i}$ 's. We have denoted the mirror D6branes with a star.

All U(1)'s of the model obtain masses except for the hypercharge. Thus, the low energy gauge group is that of the standard model $(S U(3) \times S U(2) \times Y)$. 
Generic intersecting D6-brane models are non-supersymmetric due to non-trivial intersections. To each of the intersections there are associated massive scalar fields which may be considered as "supersymmetric-partners", squarks and sleptons, of the massless chiral fermions. They have the same multiplicity $\left|I_{i j}\right|$ and carry the same gauge quantum numbers. The lightest of those states have masses

$$
\begin{aligned}
& t_{1}: \alpha^{\prime}(\text { Mass })^{2}=\frac{1}{2}\left(-\vartheta_{1}+\vartheta_{2}+\vartheta_{3}\right) \\
& t_{2}: \alpha^{\prime}(\text { Mass })^{2}=\frac{1}{2}\left(\vartheta_{1}-\vartheta_{2}+\vartheta_{3}\right) \\
& t_{3}: \quad \alpha^{\prime}(\text { Mass })^{2}=\frac{1}{2}\left(\vartheta_{1}+\vartheta_{2}-\vartheta_{3}\right)
\end{aligned}
$$

Here $\vartheta_{i}$ are the intersection angles (in units of $\pi$ ) at each of the three sub-tori. Thus, the masses depend on the moduli of the internal torus. Although in principle some of the scalars could be tachyonic, in general it is possible to vary the compact radii in order to get rid of all tachyons of a given model 90, 91.

On the D6-branes there is at the tree level, an $N=4$ SUSY gauge multiplet for each of the groups of the SM. It is expected that loop effects involving the fields at the intersections render the charged SUSY partners of the gauge bosons massive, with masses of the order of the string scale.

We also have possible Higgs fields coupling to quarks coming in four varieties with charges under $Q_{2}, Q_{1}$ and hypercharge given in table 6 .

\begin{tabular}{|c|c|c|c|}
\hline Higgs & $Q_{2}$ & $Q_{1}$ & $\mathrm{Y}$ \\
\hline \hline$h_{1}$ & 1 & -1 & $1 / 2$ \\
\hline$h_{2}$ & -1 & 1 & $-1 / 2$ \\
\hline \hline$H_{1}$ & -1 & -1 & $1 / 2$ \\
\hline$H_{2}$ & 1 & 1 & $-1 / 2$ \\
\hline
\end{tabular}

Table 6: Electroweak Higgs fields

Such Higgs fields can trigger the electroweak symmetry breaking.

There has been a lot of progress in constructing D-brane models that come quite close to the SM model. They involve non-supersymmetric intersecting brane models in a flat space [82, 84, [88]- 91, [137]- 144] or with a nontrivial internal space [145]-[151] as well as supersymmetric models, [152]-[158]. The effective low energy couplings of such models were also investigated [159]-165]. Although none so far can be claimed to be fully successful, progress in model building gives promise that fully realistic string models may be available soon. 


\section{Novel realizations of four-dimensional gravity}

We will now turn to another ingredient of fundamental interactions namely gravity. In D-brane constructions, gravity is obtained from the closed string sector and propagates in ten dimensions before compactification. Four-dimensional gravity emerges at large distance after six dimensions become compact. For future comparison we will study here the gravitational potential of a $4+\mathrm{n}$ dimensional gravity theory compactified on an n-dimensional torus with (equal) radii $R$.

$$
V\left(r, \theta_{i}\right)=\sum_{n_{i} \in Z} \frac{M^{-2-n}}{\left[r^{2}+R^{2} \sum_{i=1}^{n}\left(\theta_{i}+n_{i}\right)^{2}\right]^{\frac{n+1}{2}}}
$$

Here $V$ is the static gravitational potential of a point source located at $r=\theta_{i}=0, \mathrm{M}$ is the $(\mathrm{n}+4)$-dimensional Planck scale, $\mathrm{r}$ is the four-dimensional radial distance and $\theta_{i}, i=1,2 \cdots, n$ are angular coordinates on the internal torus with uniform period 1.

- At four-dimensional distances much smaller than the size of the internal torus gravity is $(\mathrm{n}+4)$-dimensional. The infinite sum in (6.1) is dominated by the $n_{i}=0$ term and thus

$$
V \sim \frac{M^{-2-n}}{\left[r^{2}+R^{2} \sum_{i=1}^{n}\left(\theta_{i}\right)^{2}\right]^{\frac{n+1}{2}}} \quad, \quad r<<R
$$

- On the other hand, for distances much longer than the size of the internal torus gravity becomes four-dimensional. Upon a Poisson resummation of (6.1) and then keeping the $\tilde{n}_{i}=0$ term we obtain

$$
V \sim \frac{1}{(M R)^{n}} \frac{M^{-2}}{r}, \quad r>>R
$$

which gives also the effective four-dimensional Planck scale as $M_{P}^{2}=M^{2}(M R)^{n}$ as expected.

Thus, compactification changes the behavior of gravity in the IR. The characteristic turn-over scale is the compactification scale.

\subsection{Randall-Sundrum localization}

We describe next an alternative realization of four-dimensional gravity that comes under the name of RS localization [37. Recent lectures describing in detail large dimensions and the RS universe can be found in [166, 167, 168].

We will consider the case of a five-dimensional bulk space with coordinates $x^{M}=$ $\left(y, x^{\mu}\right), \mu=0,1,2,3$. We also consider a three-brane located at $y=0$. Apart from the five-dimensional Einstein term we also have a constant energy density on the brane, and a constant energy density in the bulk. We can summarize the effective action as 


$$
S=\int d y d^{4} x \sqrt{g}\left[M^{3} R-\Lambda\right]-\delta(y) \int d^{4} \xi \sqrt{\hat{g}} V_{b}
$$

where $\hat{g}_{a b}$ is the induced metric on the brane $\hat{g}_{a b}=g_{M N} \frac{\partial x^{M}}{\partial \xi^{a}} \frac{\partial x^{N}}{\partial \xi^{b}}$. We will pick a static gauge for the brane coordinates $\xi^{a}=x^{a}$. To simplify matters we will also consider an orbifold structure under $y \rightarrow-y$. Thus, the two parts of space-time separated by the brane are mirror symmetric around the position of the brane.

We would like to solve the equations of motion stemming from the action (6.1.1). Let us first seek solutions invariant under the orbifold action which are flat along the brane, and depend only on the fifth-coordinate $y$.

The ansatz for the five-dimensional metric is

$$
d s^{2}=e^{2 A(y)} d x^{\mu} d x_{\mu}+d y^{2}
$$

In order for the flat-brane ansatz to provide a solution, the two vacuum energies must be related

$$
V_{b}^{2}=-12 \Lambda M^{3}
$$

This implies that the bulk vacuum energy $\Lambda$ must be negative. We will also define the RS (AdS) energy scale

$$
K=-\frac{\Lambda}{V_{b}}
$$

The gravitational equations have the solution

$$
A(y)=-K|y|
$$

The space on the one-side of the brane is a slice of $A d S_{5}$ patched-up with its mirror image at $y=0$. Indeed defining $r=e^{K y}$ for $y>0$ and scaling $x^{\mu} \rightarrow x^{\mu} / k$ we obtain

$$
d s^{2}=\frac{1}{K^{2} r^{2}}\left[d r^{2}+d x^{\mu} d x_{\mu}\right] \quad, \quad r \geq 1
$$

which is the $r \geq 1$ slice of $A d S_{5}$ in Poincaré coordinates with AdS energy scale $K$. Note that the orbifold has removed the boundary of $A d S_{5}$ at $r=0$.

An interesting further question concerns the effective interactions mediated by gravity in this background. To find them we must study the small fluctuations around this solution [37, 169, 170, 171]. Direct variation of the equations along the brane longitudinal directions and gauge fixing gives a scalar equation for the static graviton propagator

$$
M^{3}\left[\left(-e^{-2 A} \nabla_{x}^{2}-\partial_{y}^{2}-4 A^{\prime} \partial_{y}\right] G(x ; y)=\delta^{(3)}(x) \delta(y)\right.
$$

where we placed the source on the brane. This can be Fourier transformed along the $x^{i}$ coordinates obtaining

$$
M^{3}\left(e^{-2 A} \vec{p}^{2}-\partial_{y}^{2}-4 A^{\prime} \partial_{y}\right] G(\vec{p} ; y)=\delta(y)
$$


Changing variables to $w=e^{K|y|}$ we obtain away from $y=0$ the solution (imposing the symmetry $G(\vec{p} ; y)=G(\vec{p} ;-y)$

$$
G(\vec{p} ; y)=B w^{2} K_{2}\left(\frac{w p}{K}\right)
$$

where $p \equiv|\vec{p}|$ and $K_{2}$ is the standard Bessel function.

The multiplicative constant $B$ is fixed by the requirement that the solution satisfies the discontinuity condition

$$
\left.\frac{\partial G(\vec{p}, y)}{\partial y}\right|_{y=0+}-\left.\frac{\partial G(\vec{p}, y)}{\partial y}\right|_{y=0-}=-\frac{1}{M^{3}}
$$

obtained from (6.1.8) by integrating both sides in the interval $(-\epsilon,+\epsilon)$ and taking the limit $\epsilon \rightarrow 0$. We obtain

$$
B=-\frac{1}{2 M^{3}} \frac{1}{2 K K_{2}(p / K)+p K_{2}^{\prime}(p / K)}
$$

We may use the identity $K_{2}^{\prime}(z)+2 K_{2}(z) / z=-K_{1}(z)$ to rewrite

$$
B=\frac{1}{2 M^{3} p K_{1}\left(\frac{p}{K}\right)}
$$

We can now investigate the force mediated by the graviton fluctuations on the brane by evaluating

$$
G(\vec{p}, 0)=\frac{1}{2 M^{3} p} \frac{K_{2}\left(\frac{p}{K}\right)}{K_{1}\left(\frac{p}{K}\right)}
$$

The static gravitational potential between two unit sources on the brane (upon transforming back to configuration space) becomes

$$
\begin{gathered}
V(r)=\frac{1}{2 \pi^{2} r} \int_{0}^{\infty} p d p \sin (p r) G(\vec{p} ; 0) \\
=\frac{1}{4 \pi} \frac{K}{M^{3}} \frac{1}{r}+\delta V(r)
\end{gathered}
$$

with

$$
\delta V(r)=\frac{1}{4 \pi} \frac{K}{M^{3} r} \int_{0}^{\infty} d q \frac{K_{0}(q)}{K_{1}(q)} \sin (q r)
$$

where here $r^{2}=\vec{x}^{2}$ the spatial distance on the brane, not to be confused with radial coordinate of $A d S_{5}$ space in (6.1.6). To obtain the second equality above, we used the identity $K_{2}(z)=2 K_{1}(z) / z+K_{0}(z)$.

For $q \rightarrow \infty$ the ratio $K_{0}(q) / K_{1}(q) \rightarrow 1$ and the integral in (6.1.15) reduces to the ill-defined integral of $\sin q$ over the positive real axis. In order to evaluate 6.1.15) we multiply the integrand by $e^{-\zeta q}$, perform the integration and then take the limit $\zeta \rightarrow 0$. The integral depends on the class of regulators (see [172] for a discussion). 
For $K r \gg 1$, the strongly oscillatory behavior of $\sin (q K r)$ results in a negligible contribution to the integral from large $q$. This means that we can employ the expansion of the Bessel functions for small $q: K_{0}(\tilde{p}) / K_{1}(\tilde{p})=-\tilde{p} \log \tilde{p}+\cdots$. We obtain in this regime [37]

$$
\delta V(r) \simeq \frac{1}{8 \pi} \frac{1}{M^{3} K} \frac{1}{r^{3}} .
$$

We thus reproduce the leading and sub-leading behavior of the potential at long distances in the RS setup.

$$
V(r)=\frac{1}{4 \pi} \frac{K}{M^{3} r}\left[1+\frac{1}{2} \frac{1}{K^{2} r^{2}}+\mathcal{O}\left(\frac{1}{K^{4} r^{4}}\right)\right] \quad, \quad K r>>1
$$

Thus, at long distances gravity is four-dimensional (with sub-leading corrections). The effective four dimensional Planck scale reads $M_{P}^{2}=M^{3} / K$.

For $K r \ll 1$ the main contribution to the integral comes from large $q$, for which $K_{0}(q) / K_{1}(q)=1$. We find

$$
\delta V(r) \simeq \frac{1}{4 \pi^{2}} \frac{1}{M^{3}} \frac{1}{r^{2}} .
$$

so that the total potential is

$$
V(r)=\frac{1}{4 \pi^{2} M^{3} r^{2}}\left[1+\pi K r+\mathcal{O}\left(K^{2} r^{2} \log r\right)\right] \quad, \quad K r<<1
$$

Thus, at short distances gravity is five-dimensional [173, 172]. This is completely analogous to compactification with radius $1 / K$. The RS setup is thus an alternative mechanism to compactification for transforming five-dimensional gravity into fourdimensional at large distances.

We have neglected the scalar part of the fluctuations. This will give an extra interaction. For such an interaction to be unobservable, the extra scalar mode (the radion) should acquire a mass of the order of an $\mathrm{eV}$ or more.

\subsection{Brane Induced Gravity}

We now describe an alternative realization of four-dimensional gravity that comes under the name of Brane Induced Gravity (BIG)[51]-[55]. The idea of induced gravity has a long history [174]. The context here is however different since gravity is induced on a brane (in general a submanifold of space-time) rather than the full space-time.

We consider first the simplest case of a five-dimensional bulk with coordinates $y, x^{\mu}, \mu=0,1,2,3$. We also consider a three-brane located at $y=0$. Apart from the five-dimensional Einstein term we would like to study the effects of a four-dimensional Einstein term localized on the brane. The presence of such an (induced) term will be motivated below.

The relevant action is

$$
S=M^{3} \int d y d^{4} x \sqrt{g} R+\delta(y) M^{3} r_{c} \int d^{4} \xi \sqrt{\hat{g}} \hat{R}
$$


where $\hat{g}_{\mu \nu}$ is the induced metric on the brane and $\hat{R}$ the induced Ricci scalar. We also parameterized the coefficient of the four-dimensional term in terms of a new length scale $r_{c}$.

We are interested in the gravitational interaction, generated by the action (6.2.1), as perceived on the brane. We will evaluate first the static propagator of (6.2.1). Although there is interesting physics in the tensor structure, we will neglect it for the moment and consider instead the scalar propagator. Placing the source on the brane (at the origin) we must solve

$$
M^{3}\left(\nabla_{3}^{2}+\partial_{y}^{2}+r_{c} \delta(y) \nabla_{3}^{2}\right) G(\vec{x} ; y)=-\delta(y) \delta^{(3)}(x)
$$

Fourier transforming in the 3 -spatial coordinates $\vec{x}$ we obtain

$$
M^{3}\left(\vec{p}^{2}-\partial_{y}^{2}+r_{c} \delta(y) \vec{p}^{2}\right) G(\vec{p} ; y)=\delta(z)
$$

The solution can be found by first solving the equations away from the position of the brane, and then matching along $y=0$. The result is

$$
G(\vec{p} ; y)=\frac{e^{-|\vec{p}||y|}}{M^{3}\left(2|\vec{p}|+r_{c} \vec{p}^{2}\right)}
$$

For the source and the probe being on the brane $y=0$ the static propagator becomes

$$
G(\vec{p} ; 0)=\frac{1}{M^{3}\left(2|\vec{p}|+r_{c} \vec{p}^{2}\right)}
$$

By Fourier transforming back we obtain the static gravitational potential

$$
V(r)=\frac{1}{2 \pi^{2} r} \int_{0}^{\infty} d p p \sin p r G(\vec{p} ; 0) .
$$

where we have set $p \equiv|\vec{p}|$ for simplicity.

We are now ready to study the behavior of the gravitational force in various regimes.

- Long distances: $p<<1 / r_{c}$. Then the propagator and the potential can be approximated as

$$
G \sim \frac{1}{2 M^{3} p} \quad, \quad V(r) \sim \frac{1}{M^{3} r^{2}}
$$

This is the behavior of five-dimensional gravity with Planck scale $M$.

- Short distances: $p>>1 / r_{c}$. We obtain in this case

$$
G \sim \frac{1}{M^{3} r_{c} p} \quad, \quad V(r) \sim \frac{1}{M^{3} r_{c} r}
$$

This is the behavior of four-dimensional gravity with effective Planck scale $M_{P}^{2}=$ $M^{3} r_{c}$. 
We have thus a situation which is inverted with respect to normal compactification: four-dimensional gravity at short distance and five dimensional gravity at long distance.

If this mechanism is to play a role in realistic situations, we must tackle an immediate problem: we know from table-top to solar system to cosmological observations that gravity is four-dimensional on length scales ranging from $100 \mu \mathrm{m}$ to $10^{26} \mathrm{~m}$. In view of this there are two possibilities:

(a) The length scale $r_{c}$ is cosmologically large. We will comment below on whether this possibility can be realized in a concrete theory.

(b) The theory must also include another mechanism that forces four-dimensional gravity at long distances. For example, the extra coordinate $y$ can be compact with radius $R$.

We will now examine these two possibilities in turn.

(a) Branes appearing in superstring theories are the best controlled candidates for the (solitonic) branes we are considering. However, such branes have no tree level induced term ${ }^{4}$. We do expect though that branes with localized degrees of freedom on them will develop a localized induced gravity term due to quantum corrections. In fact in any theory, one-loop diagrams of matter fields generate a quadratically divergent correction to the Einstein term (in four dimensions). Although this calculation is tricky ${ }^{5}$ we expect that the coefficient of the induced four-dimensional Einstein term due to a given particle going around in the loop is given by $\zeta \Lambda^{2}$ where $\Lambda$ is an ultraviolet cutoff and $\zeta$ depends on the particle. If we regularize using the heat kernel method, a scalar contributes $\frac{1}{6}-\xi$ where $\xi$ is the conformal coupling, a Weyl fermion contributes $-\frac{1}{6}$, a massless vector $-\frac{2}{3}$ [174. However, the individual contributions as calculated in string theory, (where the generic cutoff is $M_{s}$ ) are different [177, 179, 180]. Such a discrepancy in gravitational contributions has been noticed before [66]. We are bound to conclude that the only reliable method to calculate such corrections is given by string theory, as other regularizations are partial, and do not imply global consistency of the gravitational theory.

In string theory, UV finiteness indicates that typically (in the absence of large values for the moduli) the one-loop correction, when non-zero, is proportional to $M_{s}^{2}$. However, KK states can transform this contribution so that it becomes moduli dependent.

In heterotic string theory and asymmetric type II vacua, such corrections are zero for $N \geq 1$ supersymmetry 176, 177, 178. In symmetric type II vacua corrections are non-zero for $N \leq 2$ supersymmetry [177. In particular, for CY compactifications, the correction is non-zero at one-loop only and is proportional to the Euler number

\footnotetext{
${ }^{4}$ Such a term can appear in D-branes of bosonic string theory 175 but its effects are masked by stringy effects. They are not visible macroscopically.

${ }^{5}$ The only gauge invariant regularization of gravity at one-loop is dimensional regularization, which is not sensitive to quadratic divergences.
} 
[179, 180, 181]. In type-I/orientifold vacua there are non-zero one-loop induced terms on D-branes when $N \leq 2$ [182, 183].

It is in principle conceivable that the induced term can be large (the transition scale $r_{c}$ is large). We can imagine for example a D-brane wrapping a small cycle 183 or a non-compact $\mathrm{CY}$ with an astronomical Euler number (that does not seem to be forbidden by geometric considerations) or a large $\mathrm{N} Z_{N}$ non-compact orbifold [184. It is not obvious that this will be stable in the absence of supersymmetry. Thus, at this point a parametrically large $r_{c}$ which is stable against radiative corrections in the absence of supersymmetry is at the speculative level.

(b) Here we have two possible contexts depending on the relation between the induced gravity scale $r_{c}$ and the compactification scale $R$.

- $r_{c}>>R$. In this case gravity is four-dimensional at all distance scales. The four-dimensional Planck constant is $M_{P}^{2}=M^{3} r_{c}$, independent of the compactification radius.

- $r_{c}<<R$ Here gravity is four-dimensional at length scales much larger than $\mathrm{R}$ or much smaller than $r_{c}$. For length scales in between, $r_{c}<<l<<R$ gravity is five dimensional. The effective four-dimensional Planck mass for $l>>R$ is $M_{P}^{2}=M^{3} R$ while for $l<<r_{c}, M_{P}^{2}=M^{3} r_{c}$.

A relevant question in case (b) is: if we need compactification why worry about BIG. The answer rests in the special properties of the coupling of graviton KK states to the brane. To understand this, consider a standard compactification with radius R. An important process is the emission of the KK states of the graviton from the brane fields. At energies small compared to the KK masses, $\sim 1 / R$, such emission is suppressed for kinematical reasons. On the other hand at energies $>>1 / R$ this emission is important, and energy is flowing from the brane to the bulk. Such processing place stringent constraints on brane-models. In order for example that the energy loss from supernovae is compatible with data an upper bound can be put on $R$.

This behavior can be understood intuitively as follows: In the regime of fourdimensional gravity $l>>R$ (or $E<<1 / R$ ) gravitons cannot easily leave the brane (otherwise gravity would have been higher-dimensional), and their emission is suppressed. In the opposite regime, $l<<R$ (or $E>>1 / R$ ) gravity is higher-dimensional and graviton emission in the bulk is unsuppressed. This intuition is applicable in more general circumstances as we will see below.

Let us now return to case (b), namely the combination of compactification with BIG. Since BIG is dominating in the UV, it suppresses the emission of KK gravitons in that regime, that would have been otherwise unsuppressed. A calculation of the value of the wave-function of graviton KK states of mass $\mathrm{m}$, in this case gives $\left|\phi_{m}(0)\right|^{2} \sim\left(4+m^{2} r_{c}^{2}\right)^{-1}$ [185]. This value is the classical coupling constant, controlling the emission of the KK states. It is obvious that it is suppressed (compared to the toroidal case $r_{c}=0$ ), for masses (energies) $>>1 / r_{c}$. This eases quite a bit the 
phenomenological constraints of such models, and greatly affects the early cosmology.

To indicate the effect of BIG, we quote that a five-to-four dimensional compactification with $R=10^{16} \mathrm{~m}$ and $R / r_{c} \sim 10^{-4}$ would not be in conflict with today's experimental data, [185]. Thus, a new dimension the size of the solar system would have been invisible!

We will now investigate BIG when there are $n>1$ transverse dimensions to the 3-brane since this case is qualitatively different.

We start again from the simplified action

$$
S=M^{n+2} \int d^{n} y d^{4} x \sqrt{g} R+\delta^{(n)}(y) M^{n+2} r_{c}^{n} \int d^{4} \xi \sqrt{\hat{g}} \hat{R}
$$

The equation for the scalar propagator in this case is

$$
M^{n+2}\left(\nabla_{x}^{2}+\nabla_{y}^{2}+r_{c}^{n} \delta^{(n)}(y) \nabla_{x}^{2}\right) G(\vec{x} ; \vec{y})=-\delta^{(n)}(y) \delta^{(3)}(x)
$$

Fourier transforming with respect to $\vec{x}$ we obtain

$$
M^{n+2}\left(\vec{p}^{2}-\nabla_{y}^{2}+r_{c}^{n} \delta^{(n)}(y) \vec{p}^{2}\right) G(\vec{p} ; \vec{y})=\delta^{(n)}(y)
$$

Going through the same steps as before we can evaluate the propagator on the brane to be

$$
G(\vec{p} ; \vec{y}=\overrightarrow{0})=\frac{D_{n}(\vec{p} ; \overrightarrow{0})}{M^{n+2}\left[1+r_{c}^{n} \vec{p}^{2} D_{n}(\vec{p} ; \overrightarrow{0})\right]}
$$

with

$$
D_{n}(\vec{p} ; \vec{y})=\int d^{n} q \frac{e^{i \vec{q} \cdot \vec{y}}}{\vec{p}^{2}+\vec{q}^{2}}
$$

In this case, since $n \geq 2, D_{n}$ is UV divergent and thus infinite. We then obtain that $G(\vec{p} ; \vec{y}=\overrightarrow{0})=1 /\left(M^{n+2} r_{c}^{n} p^{2}\right)$ which indicates four-dimensional behavior at all distances. The bulk term is completely diluted when $n \geq 2$. This however, is due to the zero thickness of the brane. We can introduce an analog of finite thickness by cutting off $D_{n}$ in the UV,

$$
D_{n}(\vec{p} ; \vec{y} ; \Lambda)=\int_{0}^{\Lambda} q^{n-1} d q d \Omega_{n-1} \frac{e^{i \vec{q} \cdot \vec{y}}}{\vec{p}^{2}+\vec{q}^{2}}
$$

To see the effects of the brane thickness we will analyse the case $n=4$ for which

$$
D_{4}(\vec{p} ; \overrightarrow{0} ; \Lambda)=\Lambda^{2}-p^{2} \log \frac{\Lambda^{2}+p^{2}}{p^{2}}+\mathcal{O}\left(\frac{1}{\Lambda^{2}}\right)
$$

so that the propagator becomes

$$
G(\vec{p} ; \overrightarrow{0}) \simeq \frac{\Lambda^{2}}{M^{6}\left(1+r_{c}^{4} \Lambda^{2} p^{2}\right)} \simeq \frac{1}{M^{6} r_{c}^{4}\left[p^{2}+\frac{1}{\Lambda^{2} r_{c}^{4}}\right]}
$$


We thus see that at distances $\Lambda^{-1}<<l<<\Lambda r_{c}^{2}$, gravity is four-dimensional. On the other hand at large distances $l>>\Lambda r_{c}^{2}$ gravity is screened and the graviton has an effective mass [183].

Even at codimension one, a brane-thickness $w$ affects the gravitational interaction. It can be shown that for distances shorter than $\sqrt{w r_{c}}$ the equivalence principle breaks down unless the theory is fine tuned [183, 186].

So far we have neglected the tensor structure of gravity. This can be taken into account and problems may arise from the scalar component of the higher-dimensional graviton. It can be shown that there is no vDVZ discontinuity in this case [188]-190. However, a new threshold scale appears where the linearized theory breaks down since the scalar graviton interactions become non-perturbative, [191, 192], although there is no consensus yet on the dependence of this scale on the four- and five-dimensional Planck scale. Further work in this direction can be found in [193-207.

\subsection{Randall-Sundrum meets Brane-Induced Gravity}

In this subsection we will investigate what happens when both mechanisms $(\mathrm{RS}+\mathrm{BIG})$ are at work simultaneously.

The relevant effective action now is (6.1.1) with the addition of the four-dimensional Einstein term localized on the brane.

$$
S=\int d y d^{4} x \sqrt{g}\left[M^{3} R-\Lambda\right]+\delta(y) \int d^{4} \xi \sqrt{\hat{g}}\left[M^{3} r_{c} \hat{R}-V_{b}\right]
$$

We will again solve the equations of motion stemming from the action (6.3.1). The crucial observation here is that since the RS solution is flat on the brane, it is not affected by the presence of the localized Einstein term. Thus with

$$
V_{b}^{2}=-12 \Lambda M^{3}
$$

the solution $6.1 .2,6.1 .5$ is still valid.

Now the equation for the static (scalar) graviton propagator is modified to

$$
M^{3}\left[\left(-e^{-2 A} \nabla_{x}^{2}-\partial_{y}^{2}-4 A^{\prime} \partial_{y}-r_{c} \delta(y) \nabla_{x}^{2}\right] G(x ; y)=\delta^{(3)}(x) \delta(y)\right.
$$

This can be Fourier-transformed along the $x^{i}$ coordinates obtaining

$$
M^{3}\left(e^{-2 A} p^{2}-\partial_{y}^{2}-4 A^{\prime} \partial_{y}+r_{c} \delta(y) p^{2}\right] G(\vec{p} ; y)=\delta(y)
$$

Changing again variables to $w=e^{K|y|}$ we obtain away from $y=0$ the solution (imposing the symmetry $G(\vec{p} ; y)=G(\vec{p} ;-y)$

$$
G(\vec{p} ; y)=B w^{2} K_{2}\left(\frac{w p}{K}\right)
$$


The multiplicative constant $B$ is fixed by the requirement on the solution to satisfy the discontinuity condition (6.1.10) from which we obtain

$$
B=\frac{1}{M^{3} p\left[2 K_{1}\left(\frac{p}{K}\right)+r_{c} p K_{2}\left(\frac{p}{K}\right)\right]}
$$

We investigate the force mediated by the graviton fluctuations on the brane by evaluating

$$
G(\vec{p}, 0)=\frac{1}{M^{3} p} \frac{K_{2}\left(\frac{p}{K}\right)}{2 K_{1}\left(\frac{p}{K}\right)+r_{c} p K_{2}\left(\frac{p}{K}\right)}
$$

The nature of the force can be directly discerned in momentum space. We have the following limits

$$
\begin{array}{ll}
\text { for } \quad p \ll K, & G(\mathbf{p}, z=0) \simeq \frac{1}{M^{3}\left(r_{c}+\frac{1}{K}\right) p^{2}}, \\
\text { for } \quad p \gg K, & G(\mathbf{p}, z=0) \simeq \frac{1}{M^{3}\left(r_{c} p^{2}+2 p\right)} .
\end{array}
$$

We shall distinguish two separate cases:

(a) Strong BIG, $K r_{c} \gg 1$ : Both for $p \ll K$, as well as for $p \gg K$ we have $G^{-1} \simeq M^{3} r_{c} p^{2}$. Thus we expect four-dimensional behavior $\sim 1 / r$ for the potential at all distances on the brane, with an effective Planck constant $M_{P l}^{2} \simeq M^{3} r_{c}$. The leading corrections to $V(r)$ can also be evaluated by employing the full propagator [172].

(b) Weak BIG $K r_{c} \ll 1$ : For $p \ll K$, we have $G^{-1} \simeq M^{3} p^{2} / K$. We find that at large distances $(r \gg 1 / K)$ the potential displays four-dimensional behavior with $\tilde{M}_{\mathrm{P}}^{2} \simeq M^{3} / K$, as in the standard RS scenario. For $k \ll p \ll 1 / r_{c}$, we have $G^{-1} \simeq 2 M^{3} p$. Thus, for distances $r_{c} \ll r \ll 1 / K$ we find a five-dimensional behavior $\sim 1 / r^{2}$ for the potential. This is in agreement with the direct evaluation of the potential for $r_{c}=0$. Finally, for $p \gg 1 / r_{c}, G^{-1} \simeq M^{3} r_{c} p^{2}$. At short distances $r \ll r_{c}$ the behavior becomes again four-dimensional $\sim 1 / r$, with $M_{P}^{2} \simeq M^{3} r_{c}$.

To summarize, the four-dimensional Einstein term induced quantum mechanically on the 3-brane affects considerably the gravitational interactions. Specifically, the gravitational potential on the brane exhibits the four-dimensional behavior $V(r) \sim 1 / r$ at all scales except in the intermediate region $r_{c} \ll r \ll 1 / K$, in which it is effectively five-dimensional and given by $V(r) \sim 1 / r^{2}$. Furthermore, for $K r_{c} \ll 1$ the strength of the gravitational interaction, i.e. the value of the effective $M_{P l}$, depends on the distance between the interacting masses. It is stronger for short distances, the ratio of its value for $r \ll r_{c}$ to the one for large $r \gg 1 / K$ being equal to $K r_{c}$. 


\subsection{Graviton emission in the bulk and brane energy-loss}

The KK spectrum of gravitons in the case of four-dimensional gravity descending from five dimensions via a combination of the RS and BIG mechanisms plays an important role in the physics. We naively expect that in energy regions where gravity is four-dimensional on the brane, KK emission will be suppressed. In the opposite case of regions of five-dimensional gravity, we expect that KK emission will be significant. We will show that this picture is correct.

We will ignore as before the tensor structure of the metric and denote by $\Phi\left(x^{\alpha}, z\right)$ its small fluctuation field around the background (6.1.2,6.1.5). More details can be found in [172]. The equation of motion at the linearized level is

$$
M^{3}\left[\frac{1}{\sqrt{-g}} \partial_{\mu}\left(\sqrt{-g} g^{\mu \nu} \partial_{\nu}\right)+\frac{r_{c}}{\sqrt{-\hat{g}}} \partial_{\alpha}\left(\sqrt{-\hat{g}} \hat{g}^{\alpha \beta} \partial_{\beta}\right)\right] \Phi\left(x^{\alpha}, z\right)=0 .
$$

As suggested by the symmetries of the background, we look for solutions in the form $\Phi\left(x^{\alpha}, z\right)=\sum_{n} \phi_{n}(z) \sigma_{n}\left(x^{\alpha}\right)$, where the $\sigma_{n}\left(x^{\alpha}\right)$ satisfy the four-dimensional KleinGordon equation $\left(\partial^{\alpha} \partial_{\alpha}+m_{n}^{2}\right) \sigma_{n}=0$. Using this in (6.4.1), one is led to the field equation

$$
\left(\partial_{z}^{2}+e^{-2 A} m_{n}^{2}+4 A^{\prime} \partial_{z}+r_{c} \delta(z) m_{n}^{2}\right) \phi_{n}(z)=0
$$

for the mode function $\phi_{n}(z)$.

The zero mode, (the solution corresponding to $m^{2}=0$ ), is not affected by the presence of the term proportional to $r_{c}$ and consequently is identical to the one in reference [37].

The KK modes, analogous to those of [37], are defined as $\psi_{n}=\exp (3 A / 2) \phi_{n}$. For $A(z)=-K|z|$ eq. (6.4.2) gives (for simplicity we omit the index $n$ from $m_{n}$ and $\left.\psi_{n}\right)$

$$
\psi(z)=N(\tilde{m}) w^{1 / 2}\left[Y_{2}(\tilde{m} w)+F(\tilde{m}) J_{2}(\tilde{m} w)\right],
$$

with $w \equiv \exp (K|z|), \tilde{m}=m / K$. The constant $F(\tilde{m})$ is fixed by the discontinuity in $\partial_{z} \phi(0)$ due to the presence of the $\delta$-function

$$
F(\tilde{m})=-\frac{2 Y_{1}(\tilde{m})+\tilde{r}_{c} \tilde{m} Y_{2}(\tilde{m})}{2 J_{1}(\tilde{m})+\tilde{r}_{c} \tilde{m} J_{2}(\tilde{m})}
$$

with $\tilde{r}_{c}=r_{c} K$.

For $w \rightarrow \infty$ the KK modes become approximate plane waves

$$
\begin{aligned}
& \psi(w) \simeq N(\tilde{m}) \sqrt{\frac{2}{\pi \tilde{m}}}\left[\sin \left(\tilde{m} w-\frac{5}{4} \pi\right)+F(\tilde{m}) \cos \left(\tilde{m} w-\frac{5}{4} \pi\right)\right] \\
& \simeq N(\tilde{m}) \sqrt{\frac{2\left(1+F^{2}(\tilde{m})\right)}{\pi \tilde{m}}} \sin \left(\tilde{m} w-\frac{5}{4} \pi+\beta(\tilde{m})\right),
\end{aligned}
$$


with $\beta=\arctan F$. As a result, for a non-compact fifth dimension, the KK modes have a continuous spectrum and their normalization is approximately that of plane waves

$$
N(\tilde{m}) \sim \sqrt{\frac{\tilde{m}}{1+F^{2}(\tilde{m})}},
$$

where we have neglected factors of order 1 . The strength of the interaction of the KK graviton modes with the other fields on the brane is determined by the square of their wave-function at the position $z=0$ of the brane. We find

$$
\psi(z=0) \sim \sqrt{\frac{\tilde{m}}{1+F^{2}(\tilde{m})}}\left[Y_{2}(\tilde{m})+F(\tilde{m}) J_{2}(\tilde{m})\right] .
$$

A careful examination of the low energy effective action reveals the presence of an additional suppression factor. The second term in the action (6.3.1) results in a non-canonical kinetic term for the fields $\sigma_{n}\left(x^{\alpha}\right)$. In order to render this term canonical we must absorb a factor $\left(1+\tilde{r}_{c}|\psi(0)|^{2}\right)^{1 / 2}$ into the redefinition of the fields.

After the KK kinetic terms have been properly normalized, the coupling of the KK modes to matter on the brane is given by $\sqrt{k / M^{3}}$. This coupling is squared in the calculation of quantities such as KK mode production rates etc. It is also accompanied by the integration over all KK states with a plane-wave measure $d m / k$. As a result, the combination $d m / M^{3}$ appears in all the estimates of KK mode production in the following.

For $\tilde{m} \ll 1$ eq. (6.4.7) gives

$$
\psi(z=0) \sim \frac{\sqrt{\tilde{m}}}{1+\tilde{r}_{c}} .
$$

We thus recover the suppression $\sim \sqrt{m / K}$ of the standard Randall-Sundrum model, which is further enhanced for large $K r_{c}$.

For $\tilde{m} \gg 1$, on the other hand, we find

$$
\psi(z=0) \sim \sin \left[\arctan \left(\frac{\tilde{r}_{c} \tilde{m}}{2}\right)-\frac{\pi}{2}\right] .
$$

For $m \gg 1 / r_{c}$ there is a significant suppression factor $\sim 1 /\left(m r_{c}\right)$, while for $m \ll 1 / r_{c}$ the wave-function on the brane is unsuppressed of order 1 .

As we will show next, these results are consistent with our naive expectation and, in addition, crucial to clarify the origin of the behavior of the effective gravitational interaction on the brane. We will again separate the two different cases: (a) Strong BIG, $K r_{c} \gg 1$, where gravity was found to be four-dimensional at all distances; (b) Weak BIG, $K r_{c} \ll 1$, where gravity again behaves as in four dimensions, except in the intermediate range $\left(K, 1 / r_{c}\right)$ where it is five-dimensional. 
(a) Strong BIG $K r_{c} \gg 1$ :

The wave function scales as

$$
\psi(0) \sim \begin{cases}\sqrt{\frac{m}{r_{c}^{2} K^{3}}}=\frac{1}{K r_{c}} \sqrt{\frac{m}{K}} & m \lesssim K \\ \frac{1}{m r_{c}}=\frac{1}{K r_{c}} \frac{K}{m}, & m \gtrsim K .\end{cases}
$$

Thus, the gravitational potential is dominated by the exchange of the zero mode and falls off $\sim 1 / r$ for all $r$. The effective squared Planck constant is $M^{3}\left(r_{c}+1 / K\right) \simeq$ $M^{3} r_{c}$.

(b) Weak BIG $K r_{c} \ll 1$ : The wave-function here scales as

$$
\psi(0) \sim \begin{cases}\sqrt{\frac{m}{K}} & m \lesssim K \\ 1, & K \lesssim m \lesssim 1 / r_{c}, \\ \frac{1}{m r_{c}}, & m \gtrsim 1 / r_{c} .\end{cases}
$$

i) For $r \gtrsim 1 / K$ the corrections to the four-dimensional potential are dominated by modes with $m \lesssim K$ because the contribution of modes with $m \gtrsim K$ is exponentially suppressed. The contribution of massive modes is negligible relative to that of the zero mode. Thus we expect a fall-off $\sim 1 / r$ with a squared Planck constant $M^{3}\left(r_{c}+1 / K\right) \simeq M^{3} / K$.

ii) For $r_{c} \lesssim r \lesssim 1 / K$ only the modes with $m \lesssim 1 / r_{c}$ contribute significantly. Those with $K \lesssim m \lesssim 1 / r_{c}$ generate a term in the potential

$$
\delta V(r) \sim \frac{1}{M^{3}} \int_{K}^{1 / r_{c}} d m \frac{e^{-m r}}{r} \psi^{2}(0) \sim \frac{1}{M^{3}} \int_{K}^{1 / r_{c}} d m \frac{e^{-m r}}{r} \simeq \frac{1}{M^{3} r^{2}} .
$$

This contribution is much larger than those from the modes with $m \lesssim K$ and the zero mode. For example the modes with $m \lesssim K$ give

$$
\delta V(r) \sim \frac{1}{M^{3}} \int_{0}^{K} d m \frac{e^{-m r}}{r} \frac{m}{K} \simeq \frac{K}{M^{3} r} .
$$

Thus, for distances $r_{c} \lesssim r \lesssim 1 / K$ we expect five-dimensional behavior $\sim 1 / r^{2}$ for the potential.

iii) Finally, at short distances $r \lesssim r_{c}$ the modes with $m \gtrsim 1 / r_{c}$ give a contribution

$$
\delta V_{1}(r) \sim \frac{1}{M^{3}} \int_{1 / r_{c}}^{\infty} d m \frac{e^{-m r}}{r} \frac{1}{m^{2} r_{c}^{2}} \simeq \frac{1}{M^{3} r_{c} r}
$$


Those with $1 / r_{c} \gtrsim m \gtrsim K$ give

$$
\delta V_{2}(r) \sim \frac{1}{M^{3}} \int_{K}^{1 / r_{c}} d m \frac{e^{-m r}}{r} \simeq \frac{1}{M^{3} r_{c} r} .
$$

These dominate over the contribution of the zero mode, as well as of the modes with $m \lesssim K$. Thus, the potential obtains again the four-dimensional form $\sim 1 / r$, with a squared Planck constant $M^{3} r_{c}$. It is remarkable that this behavior is not due to the zero mode, as one might have guessed, but instead it is attributed to the exchange of massive modes with masses $m \gtrsim K$. Similar behavior was also observed in [51]-[55, 208].

\section{The brane-universe and its cosmology}

There several ways of studying the cosmology of a brane-universe that depend largely on the formalism that describes the brane dynamics and its interaction with the bulk. There is the RS description [37, 36], appropriate from gravitating branes, that has the advantage of simplicity since many of the intricacies of the UV description are truncated. There is the probe description (mirage cosmology, [41]) which uses the DBI action to study the brane-geodesics in bulk geometries. Finally there is the BCFT description of D-branes, which for cosmological backgrounds is in its infancy and will not be treated here.

\subsection{Brane cosmological evolution in the Randall-Sundrum paradigm.}

The RS context was described (in the static case) in section 6.1. Here we will allow the brane metric to depend also on time, in order to derive effective equations for the associated cosmology.

Thus, the model is described by the five-dimensional action

$$
S=\int d^{5} x \sqrt{-g}\left(M^{3} R-\Lambda+\mathcal{L}_{B}^{m a t}\right)+\int d^{4} x \sqrt{-\hat{g}}\left(-V+\mathcal{L}_{b}^{m a t}\right),
$$

where $R$ is the curvature scalar of the five-dimensional metric $g_{A B}, A, B=0,1,2,3,5$, $\Lambda$ is the bulk cosmological constant, and $\hat{g}_{\alpha \beta}$, with $\alpha, \beta=0,1,2,3$, is the induced metric on the 3 -brane. We identify $(x, z)$ with $(x,-z)$, where $z \equiv x_{5}$. However, following the conventions of [37] we extend the bulk integration over the entire interval $(-\infty, \infty)$. The quantity $V$ includes the brane tension as well as quantum contributions to the four-dimensional cosmological constant. We have added an arbitrary matter action on the brane, as well as in the bulk for future convenience.

We consider the following cosmological ansatz for the metric

$$
d s^{2}=-n^{2}(t, z) d t^{2}+a^{2}(t, z) \gamma_{i j} d x^{i} d x^{j}+b^{2}(t, z) d z^{2},
$$


where $\gamma_{i j}$ is a maximally symmetric 3 -dimensional metric. We use $\tilde{k}$ to parameterize the spatial curvature.

The non-zero components of the five-dimensional Einstein tensor are

$$
\begin{aligned}
G_{00}= & 3\left\{\frac{\dot{a}}{a}\left(\frac{\dot{a}}{a}+\frac{\dot{b}}{b}\right)-\frac{n^{2}}{b^{2}}\left(\frac{a^{\prime \prime}}{a}+\frac{a^{\prime}}{a}\left(\frac{a^{\prime}}{a}-\frac{b^{\prime}}{b}\right)\right)+\tilde{k} \frac{n^{2}}{a^{2}}\right\}, \\
G_{i j}= & \frac{a^{2}}{b^{2}} \gamma_{i j}\left\{\frac{a^{\prime}}{a}\left(\frac{a^{\prime}}{a}+2 \frac{n^{\prime}}{n}\right)-\frac{b^{\prime}}{b}\left(\frac{n^{\prime}}{n}+2 \frac{a^{\prime}}{a}\right)+2 \frac{a^{\prime \prime}}{a}+\frac{n^{\prime \prime}}{n}\right\} \\
& +\frac{a^{2}}{n^{2}} \gamma_{i j}\left\{\frac{\dot{a}}{a}\left(-\frac{\dot{a}}{a}+2 \frac{\dot{n}}{n}\right)-2 \frac{\ddot{a}}{a}+\frac{\dot{b}}{b}\left(-2 \frac{\dot{a}}{a}+\frac{\dot{n}}{n}\right)-\frac{\ddot{b}}{b}\right\}-\tilde{k} \gamma_{i j}, \\
G_{05}= & 3\left(\frac{n^{\prime}}{n} \frac{\dot{a}}{a}+\frac{a^{\prime}}{a} \frac{\dot{b}}{b}-\frac{\dot{a}^{\prime}}{a}\right), \\
G_{55}= & 3\left\{\frac{a^{\prime}}{a}\left(\frac{a^{\prime}}{a}+\frac{n^{\prime}}{n}\right)-\frac{b^{2}}{n^{2}}\left(\frac{\dot{a}}{a}\left(\frac{\dot{a}}{a}-\frac{\dot{n}}{n}\right)+\frac{\ddot{a}}{a}\right)-\tilde{k} \frac{b^{2}}{a^{2}}\right\} .
\end{aligned}
$$

Primes indicate derivatives with respect to $z$, while dots derivatives with respect to $t$.

The five-dimensional Einstein equations take the usual form

$$
G_{A C}=\frac{1}{2 M^{3}} T_{A C},
$$

where $T_{A C}$ denotes the total energy-momentum tensor.

Assuming a perfect fluid on the brane and, possibly an additional energy-momentum $\left.T_{C}^{A}\right|_{m, B}$ in the bulk, we write

$$
\begin{aligned}
T_{C}^{A} & =\left.T_{C}^{A}\right|_{\mathrm{v}, b}+\left.T_{C}^{A}\right|_{m, b}+\left.T_{C}^{A}\right|_{\mathrm{v}, B}+\left.T_{C}^{A}\right|_{m, B} \\
\left.T_{C}^{A}\right|_{\mathrm{v}, b} & =\frac{\delta(z)}{b} \operatorname{diag}(-V,-V,-V,-V, 0) \\
\left.T_{C}^{A}\right|_{\mathrm{v}, B} & =\operatorname{diag}(-\Lambda,-\Lambda,-\Lambda,-\Lambda,-\Lambda) \\
\left.T_{C}^{A}\right|_{\mathrm{m}, b} & =\frac{\delta(z)}{b} \operatorname{diag}(-\tilde{\rho}, \tilde{p}, \tilde{p}, \tilde{p}, 0),
\end{aligned}
$$

where $\tilde{\rho}$ and $\tilde{p}$ are the energy density and pressure on the brane, respectively. The behavior of $\left.T_{C}^{A}\right|_{m, B}$ is in general complicated in the presence of flows, but we will not specify it further at this point.

We wish to solve the Einstein equations at the location of the brane following [38]- [40]. We indicate by the subscript $o$ the value of various quantities on the brane. Integrating equations (7.1.3), (7.1.4) with respect to $z$ around $z=0$, enforcing also the $Z_{2}$ symmetry, gives the jump conditions

$$
\begin{aligned}
& a_{o^{+}}^{\prime}=-a_{o^{-}}^{\prime}=-\frac{1}{12 M^{3}} b_{o} a_{o}(V+\tilde{\rho}) \\
& n_{o^{+}}^{\prime}=-n_{o^{-}}^{\prime}=\frac{1}{12 M^{3}} b_{o} n_{o}(-V+2 \tilde{\rho}+3 \tilde{p}) .
\end{aligned}
$$


The other two Einstein equations (7.1.5), (7.1.6) give

$$
\begin{gathered}
\frac{n_{o}^{\prime}}{n_{o}} \frac{\dot{a}_{o}}{a_{o}}+\frac{a_{o}^{\prime}}{a_{o}} \frac{\dot{b}_{o}}{b_{o}}-\frac{\dot{a}_{o}^{\prime}}{a_{o}}=\frac{1}{6 M^{3}} T_{05} \\
\frac{a_{o}^{\prime}}{a_{o}}\left(\frac{a_{o}^{\prime}}{a_{o}}+\frac{n_{o}^{\prime}}{n_{o}}\right)-\frac{b_{o}^{2}}{n_{o}^{2}}\left(\frac{\dot{a}_{o}}{a_{o}}\left(\frac{\dot{a}_{o}}{a_{o}}-\frac{\dot{n}_{o}}{n_{o}}\right)+\frac{\ddot{a}_{o}}{a_{o}}\right)-\tilde{k} \frac{b_{o}^{2}}{a_{o}^{2}}=-\frac{1}{6 M^{3}} \Lambda b_{o}^{2}+\frac{1}{6 M^{3}} T_{55},
\end{gathered}
$$

where $T_{05}, T_{55}$ are the 05 and 55 components of $\left.T_{A C}\right|_{m, B}$ evaluated on the brane. Substituting (7.1.12), (7.1.13) in equations (7.1.14), (7.1.15) one obtains

$$
\begin{gathered}
\dot{\tilde{\rho}}+3 \frac{\dot{a}_{o}}{a_{o}}(\tilde{\rho}+\tilde{p})=-\frac{2 n_{o}^{2}}{b_{o}} T_{5}^{0} \\
\frac{1}{n_{o}^{2}}\left(\frac{\ddot{a}_{o}}{a_{o}}+\left(\frac{\dot{a}_{o}}{a_{o}}\right)^{2}-\frac{\dot{a}_{o}}{a_{o}} \frac{\dot{n}_{o}}{n_{o}}\right)+\frac{\tilde{k}}{a_{o}^{2}}=\frac{1}{6 M^{3}}\left(\Lambda+\frac{1}{12 M^{3}} V^{2}\right) \\
-\frac{1}{144 M^{6}}(V(3 \tilde{p}-\tilde{\rho})+\tilde{\rho}(3 \tilde{p}+\rho))-\frac{1}{6 M^{3}} T_{5}^{5} .
\end{gathered}
$$

In the model that reduces to the Randall-Sundrum vacuum [37] in the absence of matter, the first term on the right hand side of equation (7.1.17) vanishes. This is the effective cosmological constant on the brane. A new mass scale $K$ may be defined through the relations $V=-\Lambda / K=12 M^{3} K$. This is the inverse characteristic length scale of AdS. Sometimes, we may keep the effective cosmological constant non-zero.

At this point we find it convenient to employ a coordinate frame in which $b_{o}=n_{o}=1$ in the above equations. This can be achieved by using Gauss normal coordinates with $b(t, z)=1$, and by going to the temporal gauge on the brane with $n_{o}=1$. The assumptions for the form of the energy-momentum tensor are then specific to this frame. Using $\beta \equiv M^{-6} / 144$ and $\gamma \equiv V M^{-6} / 144$, and omitting the subscript o for convenience in the following, we rewrite equations (7.1.16) and (7.1.17) in the equivalent first order form

$$
\begin{gathered}
\dot{\tilde{\rho}}+3(1+w) \frac{\dot{a}}{a} \tilde{\rho}=-\tilde{T} \quad, \quad \frac{\dot{a}^{2}}{a^{2}}=\beta \tilde{\rho}^{2}+2 \gamma(\tilde{\rho}+\tilde{\chi})-\frac{\tilde{k}}{a^{2}}+\tilde{\lambda} \\
\dot{\tilde{\chi}}+4 \frac{\dot{a}}{a} \tilde{\chi}=\left(\frac{\beta}{\gamma} \tilde{\rho}+1\right) \tilde{T}-\frac{1}{6 \gamma M^{3}} \frac{\dot{a}}{a} T^{5},
\end{gathered}
$$

where $\tilde{p}=w \tilde{\rho}, \tilde{T}=2 T_{5}^{0}$ is the discontinuity of the zero-five component of the bulk energy-momentum tensor, and $\tilde{\lambda}=\left(\Lambda+V^{2} / 12 M^{3}\right) / 12 M^{3}$ the effective cosmological constant on the brane.

In the equations above, Eq. (7.1.18) is the definition of the auxiliary density $\tilde{\chi}$. With this definition, the other two equations are equivalent to the original system (7.1.16,7.1.17). 
At this point we will specialize to the pure RS cosmology where the only field that lives in the bulk is the metric, and thus $\tilde{T}=T_{5}^{5}=0$. Then the cosmological equations on the brane (7.1.18), (7.1.19) become

$$
\begin{gathered}
\dot{\tilde{\rho}}+3(1+w) \frac{\dot{a}}{a} \tilde{\rho}=0 \quad, \quad \dot{\tilde{\chi}}+4 \frac{\dot{a}}{a} \tilde{\chi}=0 \\
\frac{\dot{a}^{2}}{a^{2}}=\beta \tilde{\rho}^{2}+2 \gamma(\tilde{\rho}+\tilde{\chi})-\frac{\tilde{k}}{a^{2}}+\tilde{\lambda}
\end{gathered}
$$

We define the dimensionless quantities

$$
\rho=\frac{\tilde{\rho}}{2 M^{3} K} \quad, \quad \chi=\frac{\tilde{\chi}}{2 M^{3} K} \quad, \quad \lambda=144 \frac{\tilde{\lambda}}{K^{2}}=\frac{12 \Lambda}{M^{3} K^{2}}+1
$$

as well as a rescaled cosmological time $\tau=K t / 6$. The combination $\tilde{k} / K^{2}$ is then dimensionless and by scaling $a$ we can set it to $k=0, \pm 1$. Then the cosmological equations become

$$
\begin{gathered}
\dot{\rho}+3(1+w) \frac{\dot{a}}{a} \rho=0 \quad, \quad \dot{\chi}+4 \frac{\dot{a}}{a} \chi=0 \\
\frac{\dot{a}^{2}}{a^{2}}=\rho^{2}+\rho+\chi-\frac{k}{a^{2}}+\lambda
\end{gathered}
$$

where now dots stand for derivatives with respect to $\tau$.

There are two effective energy densities that drive the cosmological evolution on the brane. The first, $\rho$, is the localized energy density on the brane, and it is conserved. The second, $\chi$ behaves like radiation, and although it affects the cosmological evolution on the brane, it is rooted in the bulk dynamics. It is the projection on the brane, of the bulk Weyl tensor [209]. It is an example of a "mirage" energy density. It is also known as dark radiation.

An extra ingredient of the cosmological evolution is the $\rho^{2}$ term in the effective Friedman equation.

For $\rho<<1$ which in dimensionfull units amounts to the localized energy density being negligible compared to the brane tension, $\tilde{\rho}<<V$, the $\rho^{2}$ term can be neglected and the cosmological evolution is four-dimensional, with only extra ingredient the mirage radiation term.

However, for $\rho>>1$ the $\rho^{2}$ term dominates and the evolution is different $H \sim \rho$, and the universe slows down. This "five-dimensional" region is analogous to the short-distance region in the static RS case in section 6.1.

There a complementary way of looking at this cosmological evolution [210 that is related to the one above by a change of coordinates. In this case the bulk metric is that of a Schwartzild-AdS black-hole in five dimensions, while now the brane is moving in a geodesic. This is in fact very similar to the mirage cosmology idea to be discussed in a subsequent section. 


\subsection{How Brane-induced gravity affects the cosmological evolution}

The Friedman equations for the RS cosmology, [38]- 40], as modified by the induced Einstein term, were derived in [211, 212]. The cosmology has been analyzed in different contexts in [230, 231, 172].

We add an induced Einstein term $M^{3} r_{c} \int d^{4} \xi \sqrt{\hat{g}} \hat{R}$ to (7.1.1). The equation (7.1.7) is modified by a localized (four-dimensional) Einstein tensor on the right hand side. This changes the Israel (jump) conditions (7.1.12,7.1.13) to

$$
\begin{aligned}
& a_{o^{+}}^{\prime}=-a_{o^{-}}^{\prime}=-\frac{1}{12 M^{3}} b_{o} a_{o}(V+\tilde{\rho})+\frac{r_{c}}{2} \frac{a_{o} b_{o}}{n_{o}^{2}}\left(\frac{\dot{a}_{o}^{2}}{a_{o}^{2}}+k \frac{n_{o}^{2}}{a_{o}^{2}}\right) \\
& n_{o^{+}}^{\prime}=-n_{o^{-}}^{\prime}=\frac{b_{o} n_{o}}{12 M^{3}}(2 \tilde{\rho}+3 \tilde{p}-V)+\frac{r_{c} b_{o}}{2 n_{o}}\left(2 \frac{\ddot{a}_{o}}{a_{o}}-\frac{\dot{a}_{o}^{2}}{a_{o}^{2}}-2 \frac{\dot{a}_{o} \dot{n}_{o}}{a_{o} n_{o}}-k \frac{n_{o}^{2}}{a_{o}^{2}}\right)(7
\end{aligned}
$$

Using (7.2.1,7.2.2) in the 05 and 55 equations, integrating once, and solving the quadratic equation for $H^{2}$ we obtain

$$
\frac{r_{c}^{2}}{2}\left(H^{2}+\frac{k}{a^{2}}\right)=1+\frac{r_{c}}{12 M^{3}}(\tilde{\rho}+V)+\epsilon \sqrt{1+\frac{r_{c}}{6 M^{3}}(\tilde{\rho}+V)-\frac{r_{c}^{2}}{12 M^{3}} \Lambda-r_{c}^{2} \chi} .
$$

where $\chi$ is the mirage radiation density related to the value of the five-dimensional Weyl tensor, and $\epsilon= \pm 1$ defining two possible branches in the solution.

For simplicity, we shall assume for the time being, that there in no significant flow of energy out of the brane through the decay of brane matter into KK modes of the graviton ${ }^{6}$. Under this assumption, the energy density $\rho_{b}$ on the brane satisfies the conservation equation

$$
\dot{\tilde{\rho}}=-3 H(\tilde{\rho}+\tilde{p}) .
$$

We are studying here the effects induced by the presence of brane matter and radiation on the RS "vacuum" background (6.1.2,6.1.5) with a given value of $K$, i.e. with the parameters $\Lambda$ and $V$ of the theory satisfying $V=-\Lambda / K=12 M^{3} K$. Using the above values for $\Lambda$ and $V$, (7.2.3) takes the form

$$
\frac{r_{c}^{2}}{2}\left(H^{2}+\frac{k}{a^{2}}\right)=1+K r_{c}+\frac{r_{c}}{12 M^{3}} \tilde{\rho}+\epsilon \sqrt{\left(1+K r_{c}\right)^{2}+\frac{r_{c}}{6 M^{3}} \tilde{\rho}-r_{c}^{2} \chi} .
$$

The equation with $\epsilon=-1$ has a smooth limit as $r_{c} \rightarrow 0$ and gives the cosmological evolution of a RS universe (7.1.21)

$$
H^{2}=\frac{V \tilde{\rho}}{72 M^{6}}+\frac{1}{4}\left(\frac{\tilde{\rho}}{6 M^{3}}\right)^{2}+\chi-\frac{k}{a^{2}} .
$$

\footnotetext{
${ }^{6}$ It will be shown later that this assumption is correct in periods of cosmological evolution where the Friedman equation is approximately four dimensional.
} 
On the contrary, the limit with vanishing bulk and brane vacuum energy is given by $K \rightarrow 0$

$$
\frac{r_{c}^{2}}{2}\left(H^{2}+\frac{k}{a^{2}}\right)=1+\frac{r_{c}}{12 M^{3}} \tilde{\rho}+\epsilon \sqrt{1+\frac{r_{c}}{6 M^{3}} \tilde{\rho}-r_{c}^{2} \chi}
$$

and provides an effective vacuum energy [230, 231] when $\epsilon=1$. From now on, for simplicity, we shall set the "mirage" radiation density to zero: $\chi=0$.

In analyzing the physical content of $(7.2 .5)$ we shall distinguish two cases.

(a) Strong BIG : $K r_{c} \gg 1$. For the static gravitational potential this corresponds to four-dimensional behavior on the brane at all scales with $M_{\mathrm{P}}^{2}=M^{3} r_{c}$. We define the dimensionless density $\hat{\rho} \equiv r_{c} \tilde{\rho} / M^{3}$. We obtain

$$
\begin{gathered}
H^{2} \simeq \frac{\tilde{\rho}}{6 M_{\mathrm{P}}{ }^{2}}-\frac{k}{a^{2}} \quad, \quad \hat{\rho} \gtrsim\left(K r_{c}\right)^{2} \\
H^{2} \simeq \frac{\tilde{\rho}}{6 M_{\mathrm{P}}{ }^{2}}-\frac{k}{a^{2}}+(\epsilon+1) \frac{2 k}{r_{c}} \quad, \quad \hat{\rho}<<\left(K r_{c}\right)^{2}
\end{gathered}
$$

Thus, for the $\epsilon=-1$ branch we obtain at all times the standard Friedman equation. For $\epsilon=1$ there is at late times a "mirage" vacuum energy $\Lambda_{4}=24 K M_{\mathrm{P}}{ }^{2} / r_{c}$ 230, 231 .

(b) Weak BIG : $K r_{c} \ll 1$. The static gravitational potential on the brane is four-dimensional at energies $E \ll K$ with $\tilde{M}_{\mathrm{P}}{ }^{2}=M^{3} / K$ (RS regime), and for $E \gg 1 / r_{c}$ with $M_{\mathrm{P}}^{2}=M^{3} r_{c}$ (Induced Gravity (IG) regime). At intermediate energies $K \ll E \ll 1 / r_{c}$ gravity is five-dimensional ( $5 d$ regime).

The Friedman equation now behaves as

$$
H^{2} \simeq \frac{\tilde{\rho}}{6 M_{\mathrm{P}}^{2}}-\frac{k}{a^{2}} \quad, \quad \hat{\rho} \gg 1
$$

corresponding to the IG regime. and

$$
H^{2} \simeq\left(\epsilon+1+K r_{c}\right) \frac{\tilde{\rho}}{6 M^{3} r_{c}}-\frac{\epsilon}{4}\left(\frac{\tilde{\rho}}{6 M^{3}}\right)^{2}-\frac{k}{a^{2}}+\frac{2(\epsilon+1)}{r_{c}^{2}}, \quad \hat{\rho} \ll 1
$$

For $\epsilon=1$ this expression becomes

$$
H^{2} \simeq \frac{\tilde{\rho}}{3 M_{\mathrm{P}}{ }^{2}}-\frac{k}{a^{2}}+\frac{4\left(1+K r_{c}\right)}{r_{c}^{2}},
$$

indicating the late-time vacuum energy. For $\epsilon=-1$, we recover the cosmology of an RS universe

$$
H^{2} \simeq \frac{\tilde{\rho}}{6 \tilde{M}_{\mathrm{P}}^{2}}+\frac{1}{4}\left(\frac{\tilde{\rho}}{6 \tilde{M}_{\mathrm{P}}^{2} K}\right)^{2}-\frac{k}{a^{2}} .
$$

We thus confirm that the rough cosmological evolution mimics the static behavior of gravity on the brane. There are further generalizations where the Gauss-Bonnet term is added in the bulk, 213]-220] which will not be discussed here. 


\subsection{Brane-bulk energy exchange}

In our discussion so far we have neglected a potentially important factor: the energy that is radiated off the brane and into the bulk in the form of KK gravitons. This can be studied as follows [172]: from the analysis of the previous section we can compute the cross-section for the emission of KK gravitons. This can be convoluted with the appropriate matter densities to provide the rate of energy loss per unit mass. This rate can be integrated to provide the full rate of energy loss as a function of time. The rate of energy loss can then be compared with the dilution of the brane energy density due to the expansion. We will describe this calculation below.

To simplify the calculations we will have to distinguish several cases.

(a)Strong BIG : $K r_{c} \gg 1$. This is the case discussed earlier in section 6.3.1. Here gravity is four dimensional at all distances and hence there are no stringent experimental constraints. All KK modes are significantly suppressed and do not affect standard processes. For example, the rate of emission of KK modes from a star can be estimated as 279

$$
\Gamma(T) \propto \frac{1}{M^{3}} \int_{0}^{T} d m \psi(0)^{2} \sim \frac{1}{M^{3}} \int_{0}^{T} d m \frac{m}{K} \frac{1}{r_{c}^{2} K^{2}} \sim \frac{1}{M_{\mathrm{P}}^{2}} \frac{T^{2}}{K^{2}} \frac{1}{r_{c} K}
$$

for $T<K$. This is much smaller than the rate of production of zero-mode gravitons $\Gamma_{0}(T) \propto 1 / M_{\mathrm{P}}{ }^{2}$, and thus it is negligible. For $T>K$ the largest contribution to the rate is

$$
\Gamma(T) \propto \frac{1}{M^{3}} \int_{K}^{T} d m \frac{1}{m^{2} r_{c}^{2}} \sim \frac{1}{M_{\mathrm{P}}^{2}} \frac{1}{r_{c} K},
$$

and is negligible again. Thus, we do not expect severe constraints on such a case from current astrophysical data, like supernovae. The only requirement is to reproduce the value of the Planck constant $M_{\mathrm{P}}{ }^{2}=M^{3} r_{c}$.

The cosmological evolution here turns out to be standard for all densities. There is a small amount of energy loss to the bulk but as we will show shortly it does not affect the Friedman evolution (7.2.8), (7.2.9) we found already in the previous section.

The change in energy density per unit time is equal to the rate of energy loss to KK gravitons per unit time and volume. For a process $a+b \rightarrow c+K K$ it is given by

$$
\left(\frac{d \rho}{d t}\right)_{\text {lost }}=-\left\langle n_{a} n_{b} \sigma_{a+b \rightarrow c+K K} v E_{K K}\right\rangle,
$$

where the brackets indicate thermal averaging. For a radiation-dominated brane we can take approximately $n_{a}, n_{b} \sim T^{3}, E_{K K} \sim T$, and estimate

$$
\left\langle\sigma_{a+b \rightarrow c+K K} v\right\rangle \sim \frac{1}{M^{3}} \int_{K}^{T} d m \frac{1}{m^{2} r_{c}^{2}} \sim \frac{1}{M_{\mathrm{P}}^{2}} \frac{1}{r_{c} K},
$$


in agreement with eq. (7.3.2). This leads to

$$
\left(\frac{d \rho}{d t}\right)_{\text {lost }} \sim-\frac{T^{7}}{M_{\mathrm{P}}^{2}} \frac{1}{r_{c} K} \sim-\frac{\rho^{7 / 4}}{M_{\mathrm{P}}^{2}} \frac{1}{r_{c} K} .
$$

We conclude that the energy loss is negligible because $(d \rho / d t)_{\text {expansion }} /(d \rho / d t)_{\text {lost }} \sim$ $\left(M_{\mathrm{P}} / \rho^{1 / 4}\right) r_{c} K \gg 1$. Thus, we obtain a standard Friedman cosmological expansion with essentially no energy loss.

(b) Weak BIG: $K r_{c} \ll 1$. Here, the deviations from the standard RS physics appear at energy scales much larger than $K$. For the gravitational potential, we expect a transition from the four-dimensional form $\sim 1 / r$ to the five-dimensional one $\sim 1 / r^{2}$ at distances $r \lesssim 1 / K$. The experimental constraints require $k \gtrsim(10 \mu \mathrm{m})^{-1} \simeq$ $10^{-11} \mathrm{GeV}$, while the value of $M$ is fixed by the relation $\tilde{M}_{\mathrm{P}}{ }^{2}=M^{3} / K$ to be $M \gtrsim 10^{9}$ $\mathrm{GeV}$.

The emission of KK modes with masses $1 / r_{c} \gtrsim m \gtrsim K$ is unsuppressed on the brane. Their contribution to various processes, such as star cooling or high-energy experiments, is analogous to those in standard toroidal compactifications, [279] with one extra dimension and a compactification radius $\sim 1 / K$. The strongest constraints arise from star cooling through the emission of KK modes.

The cosmology of this scenario has several novel features, as was indicated in the previous section. For densities $\rho \lesssim M^{3} K$ one expects the standard cosmological evolution with $H^{2} \sim \tilde{\rho} / \tilde{M}_{\mathrm{P}}{ }^{2}$ (RS regime). For $K, M$ near the lower bound set by observations $K \sim 10^{-11}, M \sim 10^{9} \mathrm{GeV}$, this regime extends up to densities $\tilde{\rho} \sim(10 \mathrm{TeV})^{4}$. However, for $M^{3} K \lesssim \tilde{\rho} \lesssim M^{3} / r_{c}$ the Hubble parameter behaves $H^{2} \sim \tilde{\rho}^{2} / M^{6}$ (5d regime), while for $\tilde{\rho} \gtrsim M^{3} / r_{c}$ we have $H^{2} \sim \tilde{\rho} / M_{\mathrm{P}}{ }^{2}$ (IG regime).

Unsuppressed emission of single $\mathrm{KK}$ gravitons can take place for the mass range $1 / r_{c}>m>K$ as shown in section 7.3.7. For a brane with energy density $\rho \gtrsim K^{4}$ it is possible to produce such unsuppressed KK gravitons that escape into the bulk. We concentrate on the case of a radiation-dominated brane-universe $\left(\tilde{\rho} \sim T^{4}\right)$, which is the most relevant for the energy scales of interest. The scale $K^{4}$ is smaller than $M^{3} K$ because we assume $K \ll M$ (otherwise the whole energy regime above $K$ is strongly coupled). We also assume $M r_{c} \gg 1$ (otherwise induced-gravity effects are masked by strong five-dimensional gravity).

The energy lost through emission of unsuppressed KK gravitons is given by eq. (7.3.3). We estimate

$$
\left\langle\sigma_{a+b \rightarrow c+K K} v\right\rangle \sim \frac{1}{M^{3}} \int_{k}^{\min \left(T, 1 / r_{c}\right)} d m=\frac{\min \left(T, 1 / r_{c}\right)}{M^{3}} .
$$

For a given temperature $T$, the energy loss is maximized if $1 / r_{c}>T$. We concentrate on this case in the following and find

$$
\left(\frac{d \rho}{d t}\right)_{\text {lost }} \sim-\frac{T^{8}}{M^{3}} \sim-\frac{\rho^{2}}{M^{3}} .
$$




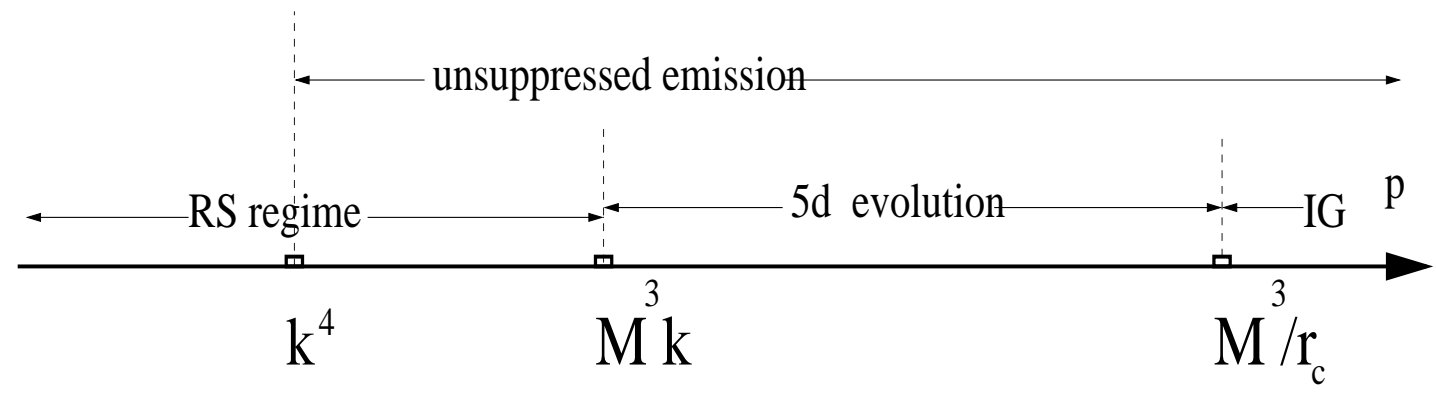

Figure 4: Different regions in density, during the evolution of the universe for $K r_{c}<<1$.

The change in energy density because of the expansion is

$$
\left(\frac{d \rho}{d t}\right)_{\exp }=-4 H \rho
$$

In the RS regime

$$
\frac{(d \rho / d t)_{\text {expansion }}}{(d \rho / d t)_{\text {lost }}} \sim \sqrt{\frac{M^{3} k}{\rho}} \gg 1
$$

Thus, the energy loss during this period is negligible compared to the dilution due to the expansion. In particular, for $M^{3} K \sim(10 \mathrm{TeV})^{4}$, the energy loss during nucleosynthesis is smaller by a factor $10^{14}$ than the rate of decrease of the energy density because of the expansion. Thus, during this period the standard cosmological evolution is not affected by energy loss. A differed way of saying this is that graviton emission is frozen-out during the RS period.

The energy loss is substantial during the $5 d$ regime, when

$$
(d \rho / d t)_{\exp } \sim-\rho^{2} / M^{3}
$$

Both $(d \rho / d t)_{\exp }$ and $(d \rho / d t)_{\text {lost }}$ are of the same order of magnitude and both lead to a decrease of the energy density for an expanding universe. Thus, it must be included in the cosmological evolution. This can be done using the metric of a radiating brane (Vadya metric) [226], 227]. We will come back to it in section 7.4.3. The energy exchange between the brane and the bulk has been studied in [221]-226], [56]-59].

\subsection{Phenomenology of brane-bulk energy exchange and its impact on brane cosmology}

We have seen in the previous section that energy exchange between the brane and the bulk, sometimes can modify significantly the Friedman cosmological evolution on the brane. It is interesting to try to investigate various patterns of such brane bulk energy exchange and its impact on the brane-world cosmology.

We will thus reconsider the general cosmological evolution equations derived in section 7.1 . 
Rescaling the variables in the basic equations (7.1.18), (7.1.19) as in (7.1.22) as well as

$$
T=\frac{\tilde{T}}{72 M^{3} K^{2}} \quad, \quad T_{5}=\frac{T^{5}{ }_{5}}{3 M^{3} K^{2}}
$$

we obtain the general equations that describe the cosmological evolution of the braneworld and its interaction with the bulk

$$
\begin{gathered}
\dot{\rho}+3(1+w) \frac{\dot{a}}{a} \rho=-T \\
\frac{\dot{a}^{2}}{a^{2}}=\rho^{2}+\rho+\chi-\frac{k}{a^{2}}+\lambda \\
\dot{\chi}+4 \frac{\dot{a}}{a} \chi=(2 \rho+1) T-\frac{\dot{a}}{a} T_{5}, \\
q=\frac{\ddot{a}}{a}=-(2+3 w) \rho^{2}-\frac{3 w+1}{2} \rho-\chi-\frac{1}{2} T_{5}+\lambda
\end{gathered}
$$

where as usual we may assume an equation of state $p=w \rho$ for the brane matter.

In (7.4.2), $T>0$ is the rate of energy outflow from the brane to the bulk, while it corresponds to inflow when negative. $T_{5}$ can be considered as the bulk back-reaction.

In order to derive solutions that are largely independent of the bulk dynamics, the $T_{5}$ term on the right hand side of the same equation must be negligible relative to the second one. This is possible if we assume that the diagonal elements of the various contributions to the energy-momentum tensor satisfy the schematic inequality [56][59]

$$
\left|\frac{\left.T\right|_{\mathrm{m}, B} ^{\text {diag }}}{\left.T\right|_{\mathrm{v}, B} ^{\text {diag }}}\right| \ll\left|\frac{\left.T\right|_{\mathrm{m}, b} ^{\text {diag }}}{\left.T\right|_{\mathrm{v}, b} ^{\text {diag }}}\right| .
$$

Our assumption is that the bulk matter relative to the bulk vacuum energy is much less important than the brane matter relative to the brane vacuum energy, at the position of the brane. In this case, the bulk is largely unperturbed by the exchange of energy with the brane. When the off-diagonal term $T_{5}^{0}$ is of the same order of magnitude or smaller than the diagonal ones, the inequality (7.4.6) implies $T \ll \rho$. Thus, under this assumption, we may neglect $T_{5}$ from (7.4.4, 7.4.5).

Even by neglecting $T_{5}$ from the equations, we do not obtain a closed system. We still have $T$ to worry about. In the previous section, we have calculated $T$ for the RS case and graviton radiation in the bulk. We have found that for periods of the evolution, $T$ is a power of the driving energy density $\rho$ on the brane. This is true for a large class of processes. Imagine that particles of the driving energy density of the brane universe (the one that dominates and drives the cosmological evolution) scatter among themselves and radiate some bulk particles via some interaction. The rate of energy loss will depend on the energy density, on cosmological temperature $\mathrm{T}$ and several particle physics parameters. using the cosmological evolution one can trade T with $\rho: \rho \sim T^{3(1+w)}$ and eventually write $T$ as a function of $\rho$ and other 
fundamental constants. If the theory on the brane is conformally invariant then $T(\rho)$ behaves like a power. Otherwise there will be scaling violations.

Motivated by this, we will parameterize $T$ as a function of the driving energy density $\rho$. The precise form depends on the details of the interaction between brane and bulk. In the scaling regime, $T$ will typically have a power dependence $T \sim \rho^{\nu}$ up to small corrections. We will use this to investigate various physical instances in the sequel.

\subsubsection{The cosmological equations for brane-bulk energy exchange}

With $T(\rho)$ a known function of $\rho$, and the approximation $T_{5} \rightarrow$ negligible is valid, the system of equations

$\dot{\rho}+3(1+w) \frac{\dot{a}}{a} \rho=-T(\rho) \quad, \quad \dot{\chi}+4 \frac{\dot{a}}{a} \chi=(2 \rho+1) T(\rho) \quad, \quad \frac{\dot{a}^{2}}{a^{2}}=\rho^{2}+\rho+\chi-\frac{k}{a^{2}}+\lambda$

is self-contained and we can attempt to find solutions. An interesting parameter of the cosmological evolution is the acceleration that can be evaluated as

$$
q=\frac{\ddot{a}}{a}=-(2+3 w) \rho^{2}-\frac{3 w+1}{2} \rho-\chi+\lambda
$$

Combining equations (7.4.1.1) we obtain

$$
a \frac{d \rho}{d a}=-3(1+w) \rho-\epsilon T(\rho)\left(\rho^{2}+\rho+\chi-\frac{k}{a^{2}}+\lambda\right)^{-1 / 2} .
$$

Similarly,

$$
a \frac{d \chi}{d a}=-4 \chi+\epsilon(2 \rho+1) T(\rho)\left(\rho^{2}+\rho+\chi-\frac{k}{a^{2}}+\lambda\right)^{-1 / 2}
$$

where $\epsilon=1$ refers to expansion, while $\epsilon=-1$ to contraction. These two equations form a two-dimensional dynamical system. The function $\chi(\rho)$ is obtained from the equation

$$
\begin{array}{r}
\left(3(1+w) \rho \sqrt{\rho^{2}+\rho+\chi-\frac{k}{a^{2}}+\lambda}+\epsilon T(\rho)\right) \frac{d \chi}{d \rho} \\
=4 \chi \sqrt{\rho^{2}+\rho+\chi-\frac{k}{a^{2}}+\lambda}-\epsilon(2 \rho+1) T(\rho) .
\end{array}
$$

Note that the equations of contraction are those of expansion with the roles of outflow and influx interchanged.

We will now set the spatial curvature on the brane-world $k=0$ and define 


$$
\hat{T}=\epsilon \rho^{-3 / 2} T \quad, \quad \zeta=\sqrt{\rho+\frac{\chi}{\rho}+1+\frac{\lambda}{\rho}}
$$

Then, the equation (7.4.1.5) becomes

$$
2 \rho \zeta^{\prime}=\frac{(1-3 w) \zeta^{2}-\hat{T} \zeta+\left[3 w-1+2(1+3 w) \rho-4 \frac{\lambda}{\rho}\right]}{3(1+w) \zeta+\hat{T}}
$$

while (7.4.2.15) becomes

$$
a \frac{d \log \rho}{d a}=-3(1+w)-\frac{\hat{T}}{\zeta}
$$

We can also derive an equation for the acceleration with the observable energy density $\rho$ as the independent variable:

$$
\begin{gathered}
-\frac{d q}{d \rho}=\frac{\left[2(1+3 w)(2+3 w) \rho^{2}+\frac{9 w^{2}-1}{2} \rho+4 \lambda-4 q\right]}{3(1+w) \rho R+\epsilon T} R+ \\
+\frac{2(3 w+1) \rho+\frac{3 w-1}{2}+\frac{1}{2} \frac{\partial T_{5}}{\partial \rho}}{3(1+w) \rho R+\epsilon T} \epsilon T
\end{gathered}
$$

where

$$
R=\sqrt{\frac{1-3 w}{2} \rho-(1+3 w) \rho^{2}+2 \lambda-q-\frac{k}{a^{2}}-\frac{T_{5}}{2}}
$$

For $k=0$, equation (7.4.1.9) simplifies to

$$
2 R^{\prime}=\frac{4 R^{2}-4 \lambda+(3 w-1) \rho+2(3 w+1) \rho^{2}}{3(1+w) \rho R+\epsilon T}
$$

\subsubsection{The four-dimensional regime}

In this regime $\rho<<1$ and the cosmological evolution in the absence of energy exchange is given by the standard Friedman equation. Equations (7.4.1.1) linearize to

$$
\dot{\rho}+3(1+w) \frac{\dot{a}}{a} \rho=-T \quad, \quad \frac{\dot{a}^{2}}{a^{2}}=\rho+\chi-\frac{k}{a^{2}}+\lambda \quad, \quad \dot{\chi}+4 \frac{\dot{a}}{a} \chi=T
$$

while the acceleration is

$$
q=\frac{\ddot{a}}{a}=-\frac{3 w+1}{2} \rho-\chi+\lambda
$$

An inspection of these equations reveals a simple interpretation. There two relevant energy densities appearing in the Friedman equation (apart from a potential vacuum energy and curvature): the energy $\rho$ of brane matter, and the mirage energy 
density (or dark radiation) $\chi$ reflecting the bulk dynamics. The function $T$ is responsible for the conversion of energy from $\rho$ to $\chi$ or vice-versa while the total energy $\rho+\chi$ is conserved:

$$
\dot{\rho}+\dot{\chi}+3 H\left[(1+w) \rho+\frac{4}{3} \chi\right]=0
$$

We can manipulate further the linearized equations to

$$
\begin{gathered}
a \frac{d \rho}{d a}=-3(1+w) \rho-\epsilon T(\rho)\left(\rho+\chi-\frac{k}{a^{2}}+\lambda\right)^{-1 / 2} \\
a \frac{d \chi}{d a}=-4 \chi+\epsilon T(\rho)\left(\rho+\chi-\frac{k}{a^{2}}+\lambda\right)^{-1 / 2} \\
\left(3(1+w) \rho \sqrt{\rho+\chi-\frac{k}{a^{2}}+\lambda}+\epsilon T(\rho)\right) \frac{d \chi}{d \rho} \\
=4 \chi \sqrt{\rho+\chi-\frac{k}{a^{2}}+\lambda}-\epsilon T(\rho) .
\end{gathered}
$$

If we now $k=0$ and define $\hat{T}=\rho^{-3 / 2} T$ and $\zeta=\sqrt{\frac{\chi}{\rho}+1+\frac{\lambda}{\rho}}$ then

$$
\begin{gathered}
2 \rho \zeta^{\prime}=\frac{(1-3 w)\left(\zeta^{2}-1\right)-\hat{T} \zeta-4 \frac{\lambda}{\rho}}{3(1+w) \zeta+\hat{T}} \\
a \frac{d \log \rho}{d a}=-3(1+w)-\frac{\hat{T}}{\zeta}
\end{gathered}
$$

\subsubsection{The exact solution for RS outflow}

Before we move on to find interesting solutions to our (approximate) cosmological equations describing energy exchange between the brane and the bulk, we will investigate an exact solution for the energy outflow from the brane in the simple RS case, that we treated perturbatively in section 7.3 .

This was given in [226, 227] and corresponds to setting in our general equations $T=a \rho^{2}$ and $T_{5}=2 a \rho^{2}$ where $a$ is a positive dimensionless constant .

We will thus consider the full system of equations (we choose $w=1 / 3$ for concreteness, so that the leading energy density on the brane is radiation, and a flat brane, $k=0$ without left-over cosmological constant, $\lambda=0$ ). We also allow (for more generality) $T=a \rho^{2}$ and $T_{5}=b \rho^{2} . b=2 a$ corresponds to a radiating $\mathrm{RS}$ brane.

The full system of equations are:

$$
\dot{\rho}+4 H \rho=-a \rho^{2} \quad, \quad \dot{\chi}+4 H \chi=a(2 \rho+1) \rho^{2}-b H \rho^{2} \quad, \quad H^{2}=\rho^{2}+\rho+\chi
$$


The linear combination $z=H+\kappa \rho$ with $4 \kappa=a-\sqrt{16+4 b+a^{2}}$ satisfies the simple equation

$$
\dot{z}=-2 z^{2} \rightarrow z=\frac{1}{2\left(t-t_{0}\right)}
$$

The general solution is given by

$$
\begin{gathered}
\rho=\frac{1}{A\left(t-t_{0}\right)\left(t-t_{1}\right)} \quad, \quad t_{1}=t_{0}+\frac{a-4 \kappa}{A} \\
\chi=\frac{\left(\kappa^{2}-1\right)+\frac{A^{2}}{4}\left(t-t_{1}\right)^{2}-A \kappa\left(t-t_{1}\right)-A\left(t-t_{0}\right)\left(t-t_{1}\right)}{A^{2}\left(t-t_{0}\right)^{2}\left(t-t_{1}\right)^{2}} \\
a(t) \sim\left(t-t_{0}\right)^{\frac{a+\sqrt{16+4 b+a^{2}}}{4 \sqrt{16+4 b+a^{2}}}}\left(t-t_{1}\right)^{\frac{\sqrt{16+4 b+a^{2}}-a}{4 \sqrt{16+4 b+a^{2}}}}
\end{gathered}
$$

From this exact solution we can study the approximation we have made earlier when we dropped the $T_{5}$ term: $H T_{5}<<(2 \rho+1) T$. To do this we define the ratio

$$
\eta \equiv \frac{H T_{5}}{(2 \rho+1) T}=\frac{b}{a} \frac{H}{(2 \rho+1)}=\frac{b}{a} \frac{A\left(t-t_{1}\right)-2 \kappa}{2\left(2+A\left(t-t_{0}\right)\left(t-t_{1}\right)\right)}
$$

As long as $\eta$ remains much smaller than one, our approximation is justified.

The general solution obtained above [227] described three types of brane-cosmological evolution:

(I) $A>0$ and $t<t_{0}$ describes a contracting universe with a big crunch at $t=t_{0}$ $\left(t_{0}<t_{1}\right)$. Here $\eta$ varies monotonically as

$$
0=\eta(-\infty) \geq \eta \geq \eta\left(t_{0}\right)=-\frac{b\left(a+\sqrt{16+4 b+a^{2}}\right)}{8 a}
$$

Here the mirage density $\chi$ is always positive if $A>4$ and switches sign at $t=$ $a /(A-4)$ if $0<A<4$.

(II) $A>0$ and $t>t_{1}$ describes an expanding universe with a big bang at $t=t_{1}$ $\left(t_{0}<t_{1}\right)$.

$$
0=\eta(\infty) \leq \eta \leq \eta\left(t_{1}\right)=\frac{b\left(-a+\sqrt{16+4 b+a^{2}}\right)}{8 a}
$$

Here $\chi$ can be always negative or switch sign .

(III) $A<0$ and $t_{1}<t<t_{0}$ which describes an expanding and recontracting universe $\left(t_{0}>t_{1}\right)$.

Here $\eta$ varies between $\eta\left(t_{0}\right)$ and $\eta\left(t_{1}\right)$. Since $\eta\left(t_{0}\right)<0$ and $\eta\left(t_{1}\right)>0$, near the big bag or big crunch $\eta$ is of order one while all the intermediate time is small

The overall message is that $\eta$ is always very small and can become of order unity or larger near a big bang or a big crunch. Moreover its value there is bounded by the coefficient of brane bulk energy exchange. 
As mentioned above, the realistic case of $\mathrm{RS}$ radiation $b=2 a$. The solution (7.4.3.3)-(7.4.3.5) simplifies to

$$
\begin{gathered}
\frac{a(t)}{a_{0}}=\left(t-t_{0}\right)^{\frac{a+2}{2(a+4)}}\left(t-t_{1}\right)^{\frac{1}{a+4}} \\
\rho=\frac{1}{A\left(t-t_{0}\right)\left(t-t_{1}\right)} \quad, \quad t_{1}=t_{0}+\frac{a+4}{A} \quad, \quad H=\rho+\frac{1}{2\left(t-t_{0}\right)} \\
\chi=H^{2}-\rho-\rho^{2}=\frac{A\left(t-t_{1}\right)-4\left(t-t_{0}\right)+4}{4 A\left(t-t_{0}\right)^{2}\left(t-t_{1}\right)}
\end{gathered}
$$

In the asymptotic 4 d regime $t \rightarrow \infty$ we have

$$
\rho \sim \frac{1}{A t^{2}} \quad, \quad \chi \sim \frac{A-4}{4 A t^{2}} \quad, \quad H \sim \frac{1}{2 t}
$$

Thus A characterizes the asymptotic ratio of energy densities.

In the $5 \mathrm{~d}$ regime $t \rightarrow t_{1}$ we have

$$
\rho \simeq H \sim \frac{1}{(a+4)\left(t-t_{1}\right)} \quad, \quad \chi \sim \frac{A-a-4}{(a+4)^{2}\left(t-t_{1}\right)}
$$

We conclude that:

In the late (4d) epoch $a \sim t^{1 / 2}$ while in the early (5d) one $a \sim t^{1 /(a+4)}$. Thus in the early epoch, outflow modifies the $t^{1 / 4}$ behavior as expected from our arguments in the previous section. Moreover, our approximation of dropping $T_{5}$ is good everywhere except close to a big crunch or a big bag.

Generally, if during the cosmological evolution $T_{5}$ remains of the same order of magnitude as $T$ for the whole period of evolution, then we have the following estimate

$$
\eta \equiv \frac{H T_{5}}{(2 \rho+1) T} \simeq \frac{H}{(2 \rho+1)}=\frac{\sqrt{\rho^{2}+\rho+\chi+\lambda}}{2 \rho+1}
$$

- If the vacuum energy is dominating the expansion then $\eta \rightarrow \sqrt{\lambda}$. If the vacuum energy is thus small in natural units, $\eta<<1$

- In regions where $\chi$ can be neglected, $\eta$ is small when $\rho<<1$ and can become at most $1 / 2$ for $\rho$ large.

\subsubsection{Inflating fixed points}

An interesting feature of the cosmological equations is the possible presence of accelerating cosmological solutions. We may look for exponential expansion with a constant Hubble parameter $H$, even if the brane content is not pure vacuum energy. We will restrict ourselves for simplicity to the $4 \mathrm{~d}$ regime. For the non-linear analysis we refer the reader to [56]-59]. 
For a fixed point, equations (7.4.2.12) must have a time-independent solution, without necessarily requiring $w=-1(\lambda=k=0)$. The possible fixed points (denoted by $*$ ) of these equations satisfy

$$
3 H_{*}(1+w) \rho_{*}=-T\left(\rho_{*}\right) \quad, \quad H_{*}^{2}=\rho_{*}+\chi_{*} \quad, \quad 4 H_{*} \chi_{*}=T\left(\rho_{*}\right) .
$$

It is clear from equation (7.4.4.1) that, for positive matter density on the brane $(\rho>0)$, flow of energy into the brane $(T(\rho)<0)$ is necessary.

The accretion of energy from the bulk depends on the dynamical mechanism that localizes particles on the brane. Its details are outside the scope of our discussion. However, it is not difficult to imagine scenaria that would lead to accretion. If the brane initially has very low energy density, energy can by transferred onto it by bulk particles such as gravitons. An equilibrium is expected to set in if the brane energy density reaches a limiting value. As a result, a physically motivated behavior for the function $T(\rho)$ is to be negative for small $\rho$ and cross zero towards positive values for larger densities. In the case of accretion it is also natural to expect that the energy transfer approaches a negative constant value for $\rho \rightarrow 0$.

The solution of equations (7.4.4.1) satisfies

$$
T\left(\rho_{*}\right)=-\frac{3}{2}(1+w) \sqrt{1-3 w} \rho_{*}^{3 / 2} \quad, \quad H_{*}^{2}=\frac{1-3 w}{4} \rho_{*} \quad, \quad \chi_{*}=-\frac{3(1+w)}{4} \rho_{*} .
$$

For a general form of $T(\rho)$ equation (7.4.4.2) is an algebraic equation with a discrete number of roots. For any value of $w$ in the region $-1<w<1 / 3$ a solution is possible. The corresponding cosmological model has a scale factor that grows exponentially with time. The energy density on the brane remains constant due to the energy flow from the bulk. This is very similar to the steady state model of cosmology [228]. The main differences are that the energy density is not spontaneously generated, and the Hubble parameter receives an additional contribution from the "mirage" density $\chi$ (see equation (7.4.4.1)).

The stability of the fixed point (7.4.4.1) determines whether the exponentially expanding solution is an attractor of neighboring cosmological flows. If we consider a small homogeneous perturbation around the fixed point $\left(\rho=\rho_{*}+\delta \rho, \chi=\chi_{*}+\delta \chi\right)$ we find that $\delta \rho, \delta \chi$ satisfy

$$
\frac{d}{d t}\left(\begin{array}{c}
\delta \rho \\
\delta \chi
\end{array}\right)=\frac{T\left(\rho_{*}\right)}{\rho_{*}} \mathcal{M}\left(\begin{array}{c}
\delta \rho \\
\delta \chi
\end{array}\right),
$$

where

$$
\begin{aligned}
\mathcal{M} & =\left(\begin{array}{cc}
-\nu+3(1-w) /(1-3 w) & 2(1-3 w) \\
\nu-2 /(1-3 w) & -2(1+9 w) /[3(1+w)(1-3 w)]
\end{array}\right) \cdot 4.4 \\
\nu & =\frac{d \ln |T|}{d \ln \rho}\left(\rho_{*}\right)
\end{aligned}
$$


and we have employed the relations (7.4.4.1) and $T(\rho) \propto \rho^{\nu}$. The eigenvalues of the matrix $\mathcal{M}$ are

$$
M_{1,2}=\frac{7+3 w-3 \nu(1+w) \pm \sqrt{24(-3+2 \nu)(1+w)+[7+3 w-3 \nu(1+w)]^{2}}}{6(1+w)}
$$

For $-1<w<1 / 3,0 \leq \nu<3 / 2$ they both have a positive real part. As we have assumed $T(\rho)<0$, the fixed point is stable in this case. The approach to the fixed-point values depends on the sign of the quantity under the square root. If this is negative the energy density oscillates with diminishing amplitude around its fixed-point value.

\subsubsection{Tracking solutions}

We will now analyze the case $\nu=3 / 2$ which lies at the boundary of the stability region discussed above. We will thus assume that $T=A \rho^{3 / 2}$, and that the universe expands and is dominated by non-relativistic matter $(\mathrm{w}=0)$. Then, in the $4 \mathrm{~d}$ regime, equation (7.4.2.18) becomes $(\lambda=0)$

$$
2 \rho \zeta^{\prime}=\frac{\zeta^{2}-A \zeta-1}{3 \zeta+A}
$$

We will parameterize the dimension-less coefficient $A$ as $A=\mu-\frac{1}{\mu} . \quad A$ is determined by the details of the microscopic cross section that gives rise to this type of energy exchange. $\mu$ running on non-negative real numbers parameterizes all pos-

sible values of $A$. When we have expansion, $A>0$ means outflow. When we have contraction $A<0$ means outflow.

The general solution of equation (7.4.5.1) is

$$
(\zeta-\mu)^{-\frac{2}{\mu}+8 \mu}\left(\zeta+\frac{1}{\mu}\right)^{-2 \mu+\frac{8}{\mu}}=C \rho^{\mu+\frac{1}{\mu}}
$$

where $C$ is a constant

Since $H^{2}=\rho+\chi$, the equation above can be re-written, in terms of $\zeta=H / \sqrt{\rho}$. Then equation (7.4.2.19) becomes

$$
\frac{a}{\rho} \frac{d \rho}{d a}=-\frac{3 \zeta+A}{\zeta}
$$

and can be integrated as a function of $a$ with the result

$$
(\zeta-\mu)^{2 \mu^{2}}\left(\zeta+\frac{1}{\mu}\right)^{2}=C^{\prime} a^{-\left(\mu^{2}+1\right)}
$$

$\rho(a)$ can be obtained by solving (7.4.5.2) and substituting into (7.4.5.4). Finally $\chi(a)=\rho\left(\zeta^{2}-1\right)$. 
We will first study a few special cases :

(i) $\mu=1$. Here we obtain

$$
\zeta^{2}=1+C \rho^{1 / 3} \Rightarrow H^{2}=\rho+C \rho^{4 / 3} \quad, \quad \rho=\frac{C^{\prime 3}}{C^{3}} \frac{1}{a^{3}} \quad, \quad \chi=\frac{C^{\prime 4}}{C^{3}} \frac{1}{a^{4}}
$$

compatible with the absence of energy exchange in this case and consequent independence of the evolution of $\rho, \chi$.

(ii) $1 / 2<\mu$. It corresponds to $-\frac{3}{2}<A$. Asymptotically $(a \rightarrow \infty)$ we obtain the tracking solution

$$
\begin{gathered}
\zeta=\mu+\tilde{C} \rho^{\frac{\mu^{2}+1}{2\left(4 \mu^{2}-1\right)}}+\ldots \quad, \quad H^{2}=\mu^{2} \rho+2 \mu \tilde{C} \rho^{\frac{\mu^{2}+1}{2\left(4 \mu^{2}-1\right)}+1}+\ldots \\
\rho \sim \tilde{C}^{\prime} a^{\frac{1}{\mu^{2}}-4}+\ldots \quad, \quad \chi=\left(\mu^{2}-1\right) \rho+\ldots
\end{gathered}
$$

Here, although the initial conditions for the real $\rho$ and mirage $\chi$ energy density are arbitrarily different (parameterized by the independent integration constants $C, C^{\prime}$ ), at late times they scale similarly with the scale factor

$$
\rho \sim \tilde{C}^{\prime} a^{\frac{1}{\mu^{2}}-4}+\ldots \quad, \quad \frac{\chi}{\rho}=\left(\mu^{2}-1\right)+\ldots
$$

Thus, the dark energy behaves as the visible energy, and such a mechanism could be used so that bulk energy simulates dark matter.

The case $-2 \leq \mu<0$ has qualitatively similar behavior. All other ranges have asymptotic $\zeta$ which is negative and thus unphysical.

Finally, in the case of outflow, there is a fixed point in the $5 \mathrm{~d}$ regime, when $A^{2}<9 / 4$ with

$$
\rho_{*}=\frac{9-4 A^{2}}{8.81} \quad, \quad H_{*}=\frac{A}{108} \sqrt{\frac{9-4 A^{2}}{2}}
$$

This is a saddle point

It is interesting to note that a similar tracking behavior has been observed in matter interacting with the dilaton in 229].

\subsubsection{Fixed points in the non-linear regime}

We will consider solutions to the non-linear system (7.4.1.1) with $H=H_{*}$ constant. The equations also imply that $\rho=\rho_{*}, T=T_{*}, \chi=\chi_{*}$ are also constant. We will see that although there may be a leftover cosmological constant $\lambda$ on the brane, the cosmological acceleration because of energy inflow, may be much smaller than $\sqrt{\lambda}$.

From the equations we obtain

$$
\rho_{*}^{ \pm}=\frac{1}{144(1+3 w)}\left[(1-3 w) \pm \sqrt{(1-3 w)^{2}+1152(1+3 w)\left(\lambda-H_{*}^{2}\right)}\right]
$$




$$
\chi_{*}=-\frac{3}{4}(1+w) \rho_{*}\left[72 \rho_{*}+1\right] \quad, \quad T_{*}=-3(1+w) H_{*} \rho_{*} \quad, \quad q_{*}=H_{*}^{2}
$$

Assuming the rate of expansion $H_{*}$ to be small compared to the cosmological constant $\lambda$, we have the following two possibilities

(i) $\lambda$ dominates in the square root. In this case $\rho_{*} \simeq \sqrt{\frac{\lambda}{18(1+3 w)}}$. There is still space for this approximation to be correct and $\rho_{*}<<1$ so that we are in the $4 \mathrm{~d}$ period.

(ii) In the opposite case $\rho_{*} \simeq \frac{1-3 w}{72(1+3 w)}$ and we can be either in the $4 \mathrm{~d}$ or the $5 \mathrm{~d}$ regime.

In either case, energy exchange can mask a leftover brane cosmological constant

\subsubsection{Other accelerating solutions}

We will present here two different families of solutions that are characteristic in their classes.

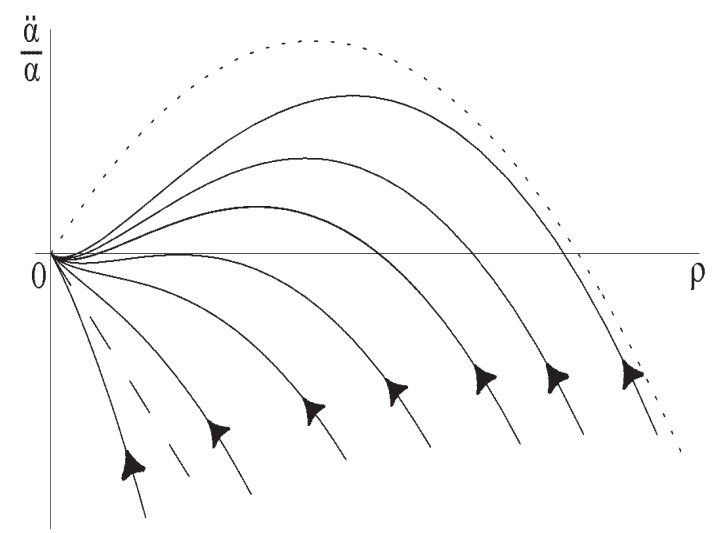

Figure 5: Outflow, $k=0, w=0, \nu=1$. The arrows show the direction of increasing scale factor

A global phase portrait of $q \equiv \ddot{a} / a$ with respect to $\rho$ during expansion in the outflow case for $k=0, w=0, \nu=1$ is shown in Figure 5. All solutions are below the limiting parabola $q<\frac{1-3 w}{2} \rho-36(1+3 w) \rho^{2}$.

One recognizes two families of solutions: The first have $q<0$ for all values of $\rho$, while the second start with a deceleration era for large $\rho$, enter an acceleration era and then return to deceleration for small enough values of $\rho$.

Solutions corresponding to initial conditions with positive $q$ (always under the limiting parabola shown with the dotted line), necessarily had a deceleration era in the past, and are going to end with an eternal deceleration era also. The straight dashed line represents the standard FRW solution without the effects of energy exchange. 
The global phase portrait of $q \equiv \ddot{a} / a$ with respect to $\rho$ during expansion for the case $k=0, w=0, \nu=1$ is shown in Figure 6. The presence of the limiting parabola as in the outflow case is apparent. However, new characteristics appear. For example, $\rho_{*}^{(-)}$attracts to eternal acceleration a whole family of solutions which start their evolution at either very low or very high densities. There is another family of solutions which are attracted to acceleration by $\rho_{*}^{(+)}$and which eventually exit to a deceleration era. Finally, there is a family of solutions, near the limiting parabola, which start with acceleration at very low densities, and eventually exit to eternal deceleration, while their density increases monotonically with time because of the influx.

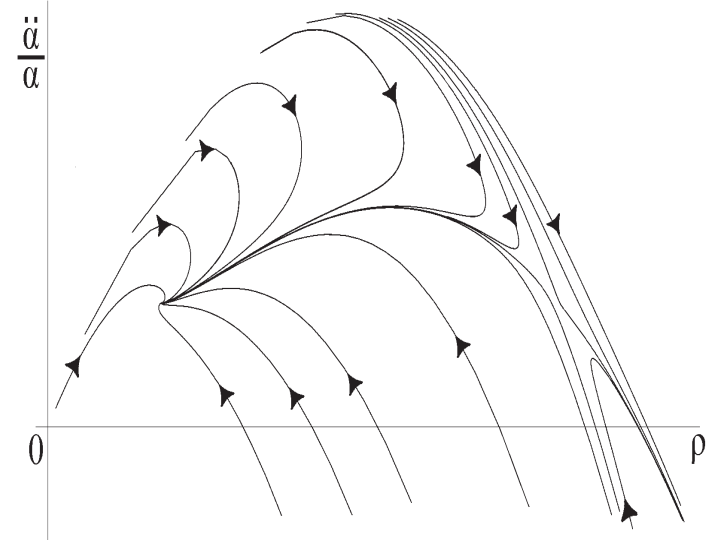

Figure 6: Influx, $k=0, w=0, \nu=1$.

For $\nu \neq 1$ one expects a different set of fixed points with varying behaviors around them. Such accelerating attractors may be relevant for the present acceleration of the universe

\subsection{Mirage Cosmology}

We have already seen that vacua of string theory are promising to describe the standard model, involve D-branes that localize the standard model particles, embedded in a ten-dimensional bulk space in which gravity propagates. This notion of braneworld, embedded in the full space-time indicates a different view (and dynamics) for cosmology. Some aspects of this have been already explored in section 7 in the context of a RS realization of gravity on a three-brane.

Here, we would like to advocate a more general approach to brane-world cosmology that is very powerful, since it is generally and easily applicable. This is the approach of mirage cosmology [41. It studies the motion of the brane world in the bulk which is affected by the energy density (and other data) on the brane-world as well as bulk fields due to other branes or just bulk geometry. An important observation in this approach [232, 233, 41] is that the motion of the brane world, via its 
interactions with the bulk fields, induces a cosmological evolution on itself, driven by brane energy densities (as in standard cosmology) as well as bulk fields (which from the brane-world point of view are interpreted as mirage energy densities). Coupled with a probe approximation for the brane-world it is powerful enough to be able to handle many non-trivial cosmological contexts.

We can thus consider our "universe" (standard model collection of almost coincident branes) in potential motion in the field of other branes carrying hidden gauge groups. Gravity as well as other universal interactions are living in the bulk space.

There are two different regimes that may be considered.

- The weak bulk field regime. In this case the matter density on the brane alone drives the cosmological expansion, in the traditional fashion.

- The strong bulk field regime. Here, the cosmological evolution of the brane is of the mirage type driven by the bulk fields and may be interpreted by the inhabitants of the brane-world as real energy, living on the brane. Detailed measurements can identify it though as mirage energy, sourced in the bulk.

An important ingredient in the physics is the induced metric on the brane which depends on the brane positions but also on brane-localized fields or energy. This dependence is at the heart of new phenomena, like variable speed of light [234 and induced cosmological evolution [41].

Mirage cosmology, by mapping cosmological evolution of the brane to geodesic motion, geometrizes the Friedman-like cosmology and gives a simple and generic pictures of accelerating, bouncing and cyclic cosmological evolution as we will explain in the sequel.

The picture of AdS/CFT correspondence and its avatars, provides a resolution of initial singularities in this context 41] which make use of the duality between gravity and gauge theory.

In order to put in context different approaches of study of time-dependent solutions of brane-worlds we will compare them in the following problem: Relative motion of two branes.

- The effective-field theory approach. One needs to calculate the effective interactions of brane-fluctuations. The solutions for the scalars parameterizing the inter-brane distance describe the relative motion of branes [235.

- The probe approach. One considers the geodesics of one of the branes moving in the fields of the other. This approach is in general approximate. In this case however, (two sets of branes) it is exact.

- Full solution of the supergravity/string equations with a time dependent solution that describes two (solitonic) branes in relative motion. This is a straightforward approach albeit very difficult in general.

Here we will focus on the second approach. The transverse space may be compact or non-compact. In the first case, when the size of the compact directions is large with respect to the string scale, we can treat most regions of brane geodesics as 
being embedded in non-compact space. There is also the possibility of non-compact transverse dimensions. In such cases, the brane-induced-gravity mechanism, introduced in previous sections, must be invoked to implement four-dimensional gravity on the branes.

Thus, the central idea is that the universe brane is moving into the bulk background fields of other branes of the theory. The motion of the brane follows, a classical geodesic in the bulk geometry. This geodesic is also affected by the matter densities' associated dynamics, localized on the brane. The prototype branes we are using here are Dp-branes. However, as it will become obvious, the results are valid for general branes, since the IR relevant parts of their world-volume actions are essentially universal.

There are two steps in the procedure:

- Determine the brane motion by solving the world-volume field equations for the scalar fields determining the position of the brane in the bulk. Other brane fields may also be excited.

- Determine the induced metric on the brane which now becomes an implicit function of time. This gives a cosmological evolution in the induced brane metric. This cosmological evolution can be reinterpreted in terms of cosmological "mirage" energy densities on the brane via a Friedman-like equation. The induced metric on the brane is the natural metric felt by the observers on the brane. We assume that our universe (SM fields) live on the brane and are made off open string fluctuations.

An important reminder here is that mirage cosmological evolution is not driven by four-dimensional gravity on the brane but by higher-dimensional gravity. One can however include the effects of induced four-dimensional gravity on the brane as we have done earlier in the RS case.

We will analyze various combinations of branes and simple background fields. They correspond to a stack of Dp-branes on and out of extremality (black Dpbranes). They provide a cosmological evolution on the probe brane that can be simulated by various types of mirage matter [41. Most prominent is radiation-types $(w=1 / 3)$ or massless scalars $(w=1)$. It should be stressed however that at small scale factor size, there are many exotic types of mirage matter including $w$ values that are outside the range $|w| \leq 1$ required by four-dimensional causality 41]. Such matter, coined phantom matter [236]-238] appears naturally here. We interpret the presence of such mirage densities as an indication that super-luminal (from that four-dimensional points of view) "shocks" are possible in such cosmologies. Super luminal signal propagation in a brane-world context have been recently pursued in 239-249. 
It has been also pointed out in 234 that the effective speed of light on the brane, in the presence of non-trivial bulk fields is in general different (lower) than that of gravitational waves. This has been shown in general in 60. Moreover, when the brane approaches horizons, the effective speed of light vanishes [234]. This was also observed later, during tachyon condensation and was coined a Carolean limit [61]. This effect indicates a similarity between unstable D-brane decay and the emergence of closed strings on one hand and confinement in the context of AdS/CFT.

Another peculiarity is that "individual" densities of mirage dilute-matter can be negative (without spoiling the overall positivity at late times).

Inflation can be produced in simple backgrounds, at the cost of breaking supersymmetry. As it was shown in 250, 253] this can be obtained from type-0 D-branes.

In mirage cosmology, the initial singularity is an artifact of the low energy description. This can be seen by studying brane motion in simple spaces like $A d S_{5} \times M$ which are globally non-singular. The induced cosmological evolution of a brane moving in such a space has a typical expansion profile due to radiation and an initial singularity (from the four-dimensional point of view). However, this singularity is an artifact. At the point of the initial singularity the universe brane joins a collection of parallel similar branes and there is (non-abelian) symmetry enhancement. The effective field theory breaks down and this gives rise to the singularity. The non-singular description is that of a non-abelian gauge theory.

An obvious question is how "real" matter/energy densities on the brane affect its geodesic motion and consequently the induced cosmological evolution. This can be studied by turning on such energy densities on the brane. We will consider as an example electromagnetic energy density and find a solution of the moving brane with a covariantly constant electric field. This gives the expected additional effect on the cosmological evolution similar to the analogous problem of radiation density in fourdimensions 41]. Although an electric field is an unrealistic cosmological background the solution we obtain is also valid when the electric energy density is thermal (and thus isotropic) in nature. This indicates that the formalism is capable of handling the most general situation possible, namely cosmological evolution driven by bulk background fields (mirage matter) as well as world-volume energy densities (real matter). Such issued have been developed further in 250-263, 266, 267.

\subsubsection{Probe-brane geodesics and induced cosmology}

According to our previous discussion, we will consider a brane-world moving in the field of a localized collection of p-branes. Here, we first review the motion of the (probe) brane-world moving in a generic static, spherically symmetric background. The brane will move in a brane-geodesic. We assume the brane to be light compared to the background so that we can neglect the back-reaction. The simplest case

corresponds to the background of a (black) Dp-brane and we will focus mostly on this case. 
The metric of a gravitating Dp-brane may be parameterized as

$$
d s_{10}^{2}=g_{00}(r) d t^{2}+g(r)(d \vec{x})^{2}+g_{r r}(r) d r^{2}+g_{S}(r) d \Omega_{8-p}
$$

and we may also generically have a dilaton $\phi(r)$ as well as a RR background $C(r)=$ $C_{0 \ldots, p}(r)$. For black Dp-branes we have

$$
\begin{gathered}
g_{00}(r)=-\frac{f(r)}{\sqrt{H_{p}(r)}}, \quad g(r)=\frac{1}{\sqrt{H_{p}(r)}}, \quad g_{r r}(r)=\frac{\sqrt{H_{p}(r)}}{f(r)} \\
g_{S}(r)=r^{2} \sqrt{H_{p}(r)}, \quad e^{\phi}=H_{p}^{(3-p) / 4} \quad, \quad C_{0, \ldots, p}=\sqrt{1+\frac{r_{0}^{7-p}}{L^{7-p}}}\left(1-\frac{1}{H_{p}}\right)
\end{gathered}
$$

and

$$
H_{p}=1+\frac{L^{(7-p)}}{r^{(7-p)}} \quad, \quad f=1-\frac{r_{0}^{(7-p)}}{r^{(7-p)}}
$$

The probe 3-brane will in general move in this background along a geodesic. Its dynamics is governed by the DBI action. In the case of maximal supersymmetry it is given by

$$
S=T_{3} \int d^{4} \xi e^{-\phi} \sqrt{-\operatorname{det}\left(\hat{G}_{\alpha \beta}+\left(2 \pi \alpha^{\prime}\right) F_{\alpha \beta}-\hat{B}_{\alpha \beta}\right)}+T_{3} \int d^{4} \xi \hat{C}_{4}+\text { anomaly terms }
$$

where we have ignored the world-volume fermions.

The embedded data are given by

$$
\hat{G}_{\alpha \beta}=G_{\mu \nu} \frac{\partial x^{\mu}}{\partial \xi^{\alpha}} \frac{\partial x^{\nu}}{\partial \xi^{\beta}} \quad, \quad \hat{B}_{\alpha \beta}=B_{\mu \nu} \frac{\partial x^{\mu}}{\partial \xi^{\alpha}} \frac{\partial x^{\nu}}{\partial \xi^{\beta}}
$$

Due to reparametrization invariance, there is a gauge freedom which may be fixed by choosing the static gauge, $x^{\alpha}=\xi^{\alpha}, \alpha=0,1,2,3$. A generic motion of the probe D3-brane will have a non-trivial angular momentum in the transverse directions as well as non-trivial momentum in the directions transverse to the probe brane but longitudinal to the background brane if $p>3$ (also known as Neumann-Dirichlet (ND) directions) In the static gauge, the relevant (bosonic) part of the brane Lagrangian reads

$$
L=e^{-\phi} \sqrt{g(r)^{3}\left[\left|g_{00}\right|-g_{r r} \dot{r}^{2}-g(r) \sum_{i=4}^{p}\left(\dot{x}^{i}\right)^{2}-g_{S}(r) h_{i j} \dot{\varphi}^{i} \dot{\varphi}^{j}\right]}-C(r) \delta_{p, 3}
$$

where $h_{i j}(\varphi) d \varphi^{i} \varphi^{j}$ is the line element of the unit (8-p)-sphere. For future purposes (generality) we will parameterize the Lagrangian as

$$
\mathcal{L}=\sqrt{A(r)-B(r) \dot{r}^{2}-\sum_{I} D_{I}(r) h_{i j}^{I} \dot{\varphi}_{I}^{i} \dot{\varphi}_{I}^{j}}-C(r) \delta_{p, 3}
$$


with

$$
A(r)=g^{3}(r)\left|g_{00}(r)\right| e^{-2 \phi} \quad, \quad B(r)=g^{3}(r) g_{r r}(r) e^{-2 \phi} \quad, \quad D_{I}(r)=g^{3}(r) g_{I}(r) e^{-2 \phi}
$$

and $C(r)$ is the RR background. The RR background only appears if the dimensions of the background and probe brane are equal. The metric $h_{i j}^{I}$ is that of $S^{d_{I}}$ while $\phi_{i}^{I}$ are the coordinates of $S^{d_{I}} . d_{I}$ can be one (relevant for compact ND directions). Several spheres can appear if the central brane is a collection of intersecting elementary branes.

In spherically symmetric backgrounds, radial motion entails non-trivial cosmological evolution on the brane with a scale factor that satisfies the mirage cosmology equations 41].

Roughly speaking the brane-world motion depends on both its charges (energy density on the brane, and RR and dilaton charge) as well as on the bulk fields, generated by the central branes. This motion induces a time-dependent effective metric on the brane and thus a cosmological evolution. In the absence of worldvolume energy distributions, homogeneity and isotropy on the brane is "explained" by the spherical symmetry of the central fields.

In the absence of 2-index antisymmetric tensor fields the effective metric on the brane-world is the induced metric. Thus, for bulk metrics of the form advocated in (7.5.1.1) the scale factor is $a^{2}=g(r)$ and its time dependence is due to the changing distance $r(t)$ from the central source.

We will now proceed further and solve for the brane-universe motion. The problem is effectively one-dimensional and can be solved by quadratures. The momenta are given by

$$
\begin{aligned}
& p_{r}=-\frac{B(r) \dot{r}}{\sqrt{A(r)-B(r) \dot{r}^{2}-\sum_{I} D_{I}(r) h_{i j}^{I} \dot{\varphi}_{I}^{i} \dot{\varphi}_{I}^{j}}} \\
& p_{i}^{I}=-\frac{D_{I}(r) h_{i j}^{I} \dot{\varphi}_{I}^{j}}{\sqrt{A(r)-B(r) \dot{r}^{2}-\sum_{I} D_{I}(r) h_{i j}^{I} \dot{\varphi}_{I}^{i} \dot{\varphi}_{I}^{j}}}
\end{aligned}
$$

The angular momenta as well as the Hamiltonian

$$
H=-E=C-\frac{A(r)}{\sqrt{A(r)-B(r) \dot{r}^{2}-\sum_{I} D_{I}(r) h_{i j}^{I} \dot{\varphi}_{I}^{i} \dot{\varphi}_{I}^{j}}}
$$

are conserved. The conserved total angular momenta in direction I are $h_{I}^{i j} p_{i}^{I} p_{j}^{I}=\ell_{I}^{2}$ and

$$
h_{i j}^{I} \dot{\varphi}_{I}^{i} \dot{\varphi}_{I}^{j}=\frac{\ell_{I}^{2}}{D_{I}^{2}}\left(A(r)-B(r) \dot{r}^{2}-\sum_{I} D_{I}(r) h_{i j}^{I} \dot{\varphi}_{I}^{i} \dot{\varphi}_{I}^{j}\right)
$$




$$
\sum_{I} D_{I} h_{i j}^{I} \dot{\varphi}_{I}^{i} \dot{\varphi}_{I}^{j}=\left(A(r)-B(r) \dot{r}^{2}\right) \frac{\sum_{I} \frac{\ell_{I}^{2}}{D_{I}}}{1+\sum_{I} \frac{\ell_{I}^{2}}{D_{I}}}
$$

The final equation for the radial variable is

$$
\sqrt{\frac{A(r)-B(r) \dot{r}^{2}}{1+\sum_{I} \frac{\ell_{I}^{2}}{D_{I}}}}=\frac{A(r)}{E+C(r)} \Rightarrow \dot{r}^{2}=\frac{A}{B}\left(1-\frac{A}{(C+E)^{2}}\left[1+\sum_{I} \frac{\ell_{I}^{2}}{D_{I}}\right]\right)
$$

and using it we can rewrite

$$
h_{i j}^{I} \dot{\varphi}_{I}^{i} \dot{\varphi}_{I}^{j}=\frac{\ell_{I}^{2}}{D_{I}^{2}} \frac{A^{2}}{(C+E)^{2}}
$$

The induced four-dimensional metric on the 3-brane universe is ${ }^{7}$

$$
d \hat{s}^{2}=\left(g_{00}+g_{r r} \dot{r}^{2}+\sum_{I} g_{I} h_{i j}^{I} \dot{\varphi}_{I}^{i} \dot{\varphi}_{I}^{j}\right) d t^{2}+g(d \vec{x})^{2}
$$

This can be brought to the standard RW form by defining cosmic time $\tau$ (coinciding with the proper brane time) as

$$
d \tau^{2}=-\left(g_{00}+g_{r r} \dot{r}^{2}+\sum_{I} g_{I} h_{i j}^{I} \dot{\varphi}_{I}^{i} \dot{\varphi}_{I}^{j}\right) d t^{2}=\frac{A^{2}}{(C+E)^{2}} \frac{e^{2 \phi}}{g^{3}} d t^{2}
$$

and the scale factor as

$$
a^{2}=g(r)
$$

Definitions (7.5.1.17,7.5.1.18) and the dynamical equation (7.5.1.14) imply that

$$
\frac{\dot{a}}{a}=\frac{1}{2} \frac{1}{g} \frac{d g}{d r} \frac{d r}{d t} \frac{d t}{d \tau}
$$

which can be written as a Friedman-like equation

$$
\left(\frac{\dot{a}(\tau)}{a(\tau)}\right)^{2}=\rho_{\mathrm{eff}}(a(\tau)) \equiv \frac{1}{4} \frac{g^{\prime 2}}{g^{2}}\left((C+E)^{2}-A\left[1+\sum_{I} \frac{\ell_{I}^{2}}{D_{I}}\right]\right) \frac{e^{-2 \phi} g^{3}}{A B}
$$

(7.5.1.20) describes the effective cosmological evolution on the brane-universe. As advocated in [267], more detailed information about the (local) physics of the brane can be recovered from the brane action and its coupling to the bulk fields.

\footnotetext{
${ }^{7}$ We assume here that $B_{\mu \nu}=0$ for D-branes. In the opposite case there are modifications to the effective metric that are interesting but will not be further discussed here. An example of mirage cosmology in the presence of nontrivial $B_{\mu \nu}=0$ was presented in [41] .
} 


\subsubsection{Orbits, bounces and static universes}

At this point and without further calculation we can qualitatively describe the connection between different brane-universe geodesics and the (effective) cosmological type of expansion or contraction perceived on the brane-universe. The function $g(r)$ linking the distance to the effective scale-factor of the brane-universe is typically a monotonic function of $r$. We ignore here the potential effect of brane-energy densities over and above the (BPS) negative tension and RR charges. Their effects will be discussed later on.

The eventual type of cosmology (behavior of the scale factor) depends on two distinct ingredients [264]-269]:

(a) The dependence of the scale factor on the radial distance $r$ of the universe brane from the center of the bulk distribution, encapsulated in the relation $a^{2}=g(r)$. There are two distinct possibilities here: In asymptotically flat bulk configurations, $a(r)$ is a monotonic function with a maximum. For non-asymptotically flat configurations (like AdS) $a(r)$ is monotonic without a bound.

(b) The type of orbit in transverse space. Such orbits are of the following form:

(i) Unbounded (hyperbolic) orbits. These are "scattering-type" orbits where the brane starts at infinite distance approaches to a minimal distance and finally retreats back to infinity. The associated behavior of the brane cosmology is then as follows: The universe is contracting until it reaches a minimum size. It subsequently (and smoothly) bounces back to an ever expanding phase. No cosmological singularity will be ever perceived on the brane-universe in this case. In the asymptotically flat case, the ever expanding phase can saturate asymptotically due to the bound in $a(r)$.

(ii) Bounded (parabolic) orbits. These are bound orbits where the distance to the central point (hyper-galaxy) oscillates between a minimum and a maximum value. The associated cosmology is cyclic alternating between expanding and contracting phases with no cosmological singularity. A very special case is that of circular orbits that corresponds to a static brane-universe. As it will be expanded upon later, the speed of light on the brane is smaller than the one in the bulk [234, 265]. At large distances, one deals with Newtonian potentials that behave like $r^{2-d}$ where $d$ is the number of transverse directions. It is well known that bound orbits exist only when the centrifugal potential (always behaving like $r^{-2}$ independent of dimension) can balance the Newtonian one. This can only happen when the Newtonian attractive potential behaves as $r^{-1}$ or $r^{-2}$.

(iii) "absorption" or "emission" orbits. These are orbits where the universe brane finally enters the horizon of the black brane (central hyper-galactic black brane) or it is "emitted" and moves outwards. We should distinguish here the following two subcases.

(iiia) Bound absorption or emission orbits. These obits originate or end at 
the horizon but they never reach infinity. They correspond to a cosmology of an expanding universe (outward motion) which reaches a maximum size, then recontracts and has a final singularity associated to the brane falling at the center $r=0$. It has been argued [41] that this singularity is resolved in the D-brane description by passing to the enhanced non-abelian theory of the full system. Nonetheless, this encounter is catastrophic for the brane-matter (and its inhabitants) since it entails a complete rearrangement of the effective matter theory similar to that of phase transition of symmetry restoration. It has been argued that already at the crossing of the horizon the brane thermalizes with the Hawking heat-bath associated with the central black brane 270, 271] provided it is colder than the black brane ${ }^{8}$. The time reversed situation involves an expanding universe with an apparent (initial) singularity in its past.

(iiib) Unbounded absorption or emission orbits. Such orbits start above the horizon and reach infinity, or start at infinity and fall through the horizon. They correspond to always expanding cosmologies with an initial singularity or always contracting cosmologies with a final singularity. The scale factor can also saturate here in asymptotically flat bulk geometries. The same remarks as above apply to the resolution of the cosmological singularity.

\subsubsection{A partial survey of various induced mirage cosmologies}

Here we will take a closer look at the various orbits. We consider a 3-brane moving in the background of a black p-brane. The internal charges that are relevant are the angular momentum $l_{s}$ on $S^{8-p}$ transverse to the p-brane as well as the momenta $l_{i}$, $i=1,2, \cdots, p-3$ along the longitudinal directions of the black p-brane that are transverse to the 3-brane universe. The relevant functions are

$$
A=f H_{p}^{\frac{p-7}{2}} \quad, \quad B=\frac{1}{f} H_{p}^{\frac{p-5}{2}} \quad, \quad D_{i}=H_{p}^{\frac{p-7}{2}} \quad, \quad D_{s}=r^{2} H_{p}^{\frac{p-5}{2}}
$$

In the special case $p=3$ there is also a $\mathrm{RR}$ potential $C=\sqrt{1+\frac{r_{0}^{4}}{L^{4}}} \frac{L^{4}}{L^{4}+r^{4}}$. We will first describe the case $p>3$.

The orbit is given by the solution of

$$
\dot{r}^{2}=\frac{f^{2}}{H_{p}}\left[1-\frac{f}{E^{2}}\left(\frac{1}{H_{p}^{\frac{7-p}{2}}}+\frac{l_{s}^{2}}{r^{2} H_{p}}+\sum_{i} l_{i}^{2}\right)\right]
$$

where $l_{s}$ is the angular momentum on the $S^{8-p}$ and $l_{i}$ are angular momenta on the Neumann-Dirichlet $S^{1}$ s. We will scale $r, t$ and $l_{s}$ appropriately so as to set the length $L$ to one.

The radius-scale factor relation is

$$
r=\left(\frac{a^{4}}{1-a^{4}}\right)^{\frac{1}{7-p}}
$$

\footnotetext{
${ }^{8}$ See also 62 for a related view.
} 
Note that here the scale factor is bounded above by 1. This can be rescaled to any desirable finite value by a choice of units. The associated Friedman equations take the form

$$
\begin{aligned}
\left(\frac{\dot{a}}{a}\right)^{2}= & \frac{(p-7)^{2}}{16} a^{2 \frac{3-p}{7-p}}\left(1-a^{4}\right)^{2 \frac{8-p}{7-p}}\left[\frac{\left(C(a) \delta_{p, 3}+E\right)^{2}}{a^{2(7-p)}}-\right. \\
& \left.-f_{p}(a)\left(1+\frac{\sum_{i} l_{i}^{2}}{a^{2(7-p)}}+\frac{l_{s}^{2}\left(1-a^{4}\right)^{\frac{2}{7-p}}}{a^{2 \frac{(p-6)^{2}+3}{7-p}}}\right)\right]
\end{aligned}
$$

with

$$
C(a)=\sqrt{1+r_{0}^{4}}\left(1-a^{4}\right) \quad, \quad f_{p}(a)=1+r_{0}^{7-p}-\frac{r_{0}^{7-p}}{a^{4}}
$$

To discuss the behavior of orbits we must study the effective potential

$$
V_{\mathrm{eff}}=\frac{f^{2}}{H_{p}}\left[1-\frac{f}{E^{2}}\left(\frac{1}{H_{p}^{\frac{7-p}{2}}}+\frac{l_{s}^{2}}{r^{2} H_{p}}+\sum_{i} l_{i}^{2}\right)\right]
$$

Zeros of the effective potential signal a turning point in the radial motion. At the horizon $r=r_{0}$ the effective potential always vanishes

$$
V_{\text {eff }}=\frac{(7-p)^{2}}{r_{0}^{p-5}\left(r_{0}^{7-p}+1\right)}\left(r-r_{0}\right)^{2}+\mathcal{O}\left(\left(r-r_{0}\right)^{3}\right)
$$

This zero is not a turning point. If no other zero $r_{*}>r_{0}$ exists, then the brane crosses eventually the horizon. Moreover $V_{\text {eff }}$ remains positive for $r$ approaching $r_{0}$ from above.

The asymptotic form of the effective potential at large $r$ is

$$
V_{\mathrm{eff}}=1-\frac{\sum_{i} l_{i}^{2}+1}{E^{2}}+\mathcal{O}\left(\frac{1}{r}\right)
$$

where $3<p<7$.

We distinguish the following cases

- (A) $E^{2}>\sum_{i} l_{i}^{2}+1$. In this case $V_{\text {eff }}(\infty)>0$ and there is always the possibility of unbounded motion. If there are no non-trivial zeros, then the possible motion is of type (iiib) in the nomenclature of the previous section (unbounded emission or absorbtion orbits).

If there are non-trivial zeros they must appear in pairs. In this case there are type (i) unbounded orbits that bounce at the largest non-trivial zero. There is always also type (iiia) orbits between the lowest non-trivial zero and the horizon. Finally, if there are more than two non-trivial zeros, there are type (ii) bound orbits corresponding to oscillating universes. 
- (B) $E^{2}<\sum_{i} l_{i}^{2}+1$. In this case $V_{\text {eff }}(\infty)<0$ and there is no unbounded motion. In this case the number of non-trivial zeros is odd. There is always a type (iiia) motion between the lowest non-trivial zero and the horizon.

If there are more than one non-trivial zeros then there are always type (ii) orbits leading to oscillating universes.

- (C) $E^{2}=\sum_{i} l_{i}^{2}+1$. This is a marginal case with $V_{\text {eff }}(\infty)=0$. We must investigate whether $V_{\text {eff }}$ vanishes from positive or negative values.

This crucially depends on $p$. For $p=4$

$$
V_{\text {eff }}=-\frac{l_{s}^{2}}{E^{2}} \frac{1}{r^{2}}+\mathcal{O}\left(\frac{1}{r^{3}}\right)
$$

When $l_{s} \neq 0$ the orbits are as in case $(\mathbf{B})$. When $l_{s}=0$

$$
V_{\text {eff }}=\frac{3+2 E^{2} r_{0}^{3}}{2 E^{2}} \frac{1}{r^{3}}+\mathcal{O}\left(\frac{1}{r^{6}}\right)
$$

and the orbits are those of case $(\mathbf{A})$

For $p=5$

$$
V_{\text {eff }}=\frac{r_{0}^{2} E^{2}-l_{s}^{2}+1}{E^{2}} \frac{1}{r^{2}}+\mathcal{O}\left(\frac{1}{r^{4}}\right)
$$

If $r_{0}^{2} E^{2}+1>l_{s}^{2}$ we have orbits similar to case $(\mathbf{A})$ while in the opposite case $r_{0}^{2} E^{2}+1<l_{s}^{2}$ we have orbits like case (B) In the marginal case $l_{s}^{2}=r_{0}^{2} E^{2}+1$

$$
V_{\text {eff }}=r_{0}^{2}\left(1+r_{0}^{2}\right) \frac{1}{r^{4}}+\mathcal{O}\left(\frac{1}{r^{6}}\right)
$$

and the orbits are of case $(\mathbf{A})$.

For $p=6$

$$
V_{\mathrm{eff}}=\frac{2 r_{0} E^{2}+1}{2 E^{2}} \frac{1}{r}+\mathcal{O}\left(\frac{1}{r^{2}}\right)
$$

and we have obits like case $(\mathbf{A})$.

The case of the black-D3-brane background is described by the following effective potential.

$V_{\text {eff;D3 }}=\frac{f^{2}}{H_{3}}\left[1-\frac{f}{(E+C)^{2}}\left(\frac{1}{H_{3}^{2}}+\sum_{i} l_{i}^{2}+\frac{l_{s}^{2}}{r^{2} H_{3}}\right)\right]=1-\frac{\sum_{i} l_{i}^{2}+1}{E^{2}}-\frac{l_{s}^{2}}{E^{2}}+\mathcal{O}\left(\frac{1}{r^{4}}\right)$

The presence of the RR 4-form appears first at order $r^{-4}$. The general structure of orbits is similar to the $p=4$ case.

Note that in all the above situations, unbounded orbits in $r$ - space correspond to universes with a scale factor $a \rightarrow 1$ in the asymptotic past and future. 


\subsubsection{Near-horizon region}

We will investigate here the near-horizon region of black Dp-branes. The effective potential is given by

$$
V_{\mathrm{eff}}=f^{2} r^{7-p}\left[1-\frac{f}{\left(E+\delta_{p, 3} r^{4}\right)^{2}}\left(r^{\frac{(7-p)^{2}}{2}}+l_{s}^{2} r^{5-p}+\sum_{i} l_{i}^{2}\right)\right]
$$

The radius-scale relation is given by

$$
r=a^{\frac{4}{7-p}}
$$

and the effective Friedmann equation reads 41

$$
\left(\frac{\dot{a}}{a}\right)^{2}=\frac{(p-7)^{2}}{16} a^{2 \frac{3-p}{7-p}}\left[\frac{\left(C(a) \delta_{p, 3}+E\right)^{2}}{a^{2(7-p)}}-f_{p}(a)\left(1+\frac{\sum_{i} l_{i}^{2}}{a^{2(7-p)}}+\frac{l_{s}^{2}}{a^{2 \frac{(p-6)^{2}+3}{7-p}}}\right)\right]
$$

with

$$
C(a)=a^{4} \quad, \quad f_{p}(a)=1-\frac{r_{0}^{7-p}}{a^{4}}
$$

The behavior of the effective potential at the horizon is the same as in (7.5.3.7) and similar remarks apply. At large $r$ we obtain that for all $3<p<7$ the effective potential reaches $-\infty$. Consequently we have only bound orbits of (ii) and (iiia) type. The maximal radial distance of such orbits grows with the energy $E$ of the motion roughly as $r_{\max }^{7-p} \simeq E^{4}$.

The case $p=3$ is special. The asymptotic form of the effective potential is here

$$
V_{\text {eff }}=2 E+r_{0}^{4}-\frac{l_{s}^{2}}{r^{2}}+\mathcal{O}\left(\frac{1}{r^{4}}\right)
$$

- $2 E+r_{0}^{4}>0$. Here we have generically unbounded orbits.

- $2 E+r_{0}^{4}<0$. Here we have bound orbits.

- $2 E+r_{0}^{4}=0$. The orbits are bound.

\subsubsection{Static universe solutions}

Fixed $r$ orbits correspond to static universes with a constant speed of light. Using $\dot{r}=0$ and the equations of motion we obtain

$$
d \hat{s}^{2}=\frac{g_{00}(r)}{1+\sum_{I} \frac{\ell_{I}^{2}}{D_{I}}} d t^{2}+g(r)(d \vec{x})^{2}
$$


from which we can read the effective light velocity as

$$
c_{\mathrm{eff}}^{2}=\frac{g_{00}}{g\left(1+\sum_{I} \frac{\ell_{I}^{2}}{D_{I}}\right)}=\frac{f}{1+\sum_{I} \frac{\ell_{I}^{2}}{D_{I}}} \leq 1
$$

where the second equality holds for black D-branes. The numerator comes from the presence of the horizon in the background brane [234] and the denominator is due to the circular motion in transverse space ${ }^{9}$.

We will now discuss orbits for $D_{p>3}$ backgrounds. In this case

$$
A=f H_{p}^{(p-7) / 2} \quad, \quad B=\frac{H_{p}^{(p-5) / 2}}{f} \quad, \quad D_{N D}=H_{p}^{(p-7) / 2} \quad, \quad D_{T}=r^{2} H_{p}^{(p-5) / 2}
$$

where $D_{N D}$ corresponds to the Neumann-Dirichlet directions and $D_{T}$ corresponds to the (8-p)-sphere. The energy condition for circular orbits at $r=r_{*}$ implies

$$
\frac{E^{2}}{A}=1+\sum_{I} \frac{\ell_{I}^{2}}{D_{I}}
$$

while the no-force condition

$$
\frac{\partial}{\partial r} \log \left[A\left(1+\sum_{I} \frac{\ell_{I}^{2}}{D_{I}}\right)\right]=\frac{\partial}{\partial r} \log \left[f H_{p}^{(p-7) / 2}\left(1+\ell_{N D}^{2} H_{p}^{(7-p) / 2}+\frac{\ell_{T}^{2}}{r^{2}} H_{p}^{(5-p) / 2}\right)\right]=0
$$

Using the above, the effective light velocity becomes

$$
c_{\mathrm{eff}}^{2}=\frac{f^{2} H_{p}^{(p-7) / 2}}{E^{2}}=\frac{f}{1+\sum_{I} \frac{\ell_{I}^{2}}{D_{I}}}
$$

It is implied that as $r_{*} \rightarrow \infty c_{\text {eff }} \rightarrow 1 /|E|$.

It is easy to argue that the existence of stable circular orbits for a range of parameters is possible only if the "gravitational" attraction can be balanced by the centrifugal force which always falls like $r^{-2}$. This can happen only if the attraction behaves as $r^{-1}$ or $r^{-2}$ and this singles out the cases of $p=5,6$

In these cases we always have stable circular orbits at any radius by appropriately adjusting the energy and angular momenta. Analyzing the dynamics of small perturbations around the circular motion we obtain the condition for stability:

$$
\frac{1}{2}\left(A^{\prime \prime}-\sum_{I} D_{I}^{\prime \prime} \frac{\ell_{I}^{2}}{D_{I}^{2}} \frac{A^{2}}{E^{2}}\right)+\sum_{I, J} B_{I} M_{I J}^{-1} B_{J} \geq 0
$$

where

$$
M_{I J}=\left(D_{I} \delta_{I J}+\ell_{I} \ell_{J}\right) \quad, \quad B_{I}=\frac{D_{I}^{\prime}}{D_{I}} \ell_{I}
$$

\footnotetext{
${ }^{9}$ This term is missing in 265.
} 
We will now examine the two relevant cases.

$p=5$. In this case there are stable circular orbits for a wide range of parameters. In the special case $r_{0}=0, L=\ell_{T}$ and there is a stable orbit for any r. Here

$$
c_{\text {eff }}^{2}=\frac{1}{\left(1+\ell_{N D}^{2}\right)\left(1+\frac{L^{2}}{r^{2}}\right)}
$$

and varies between zero and $1 /\left(1+\ell_{N D}^{2}\right)$ for various orbits. In the general case, $r_{0}>0$, there are stable circular orbits provided $\ell_{T}^{2}>L^{2}+r_{0}^{2}\left(1+\ell_{N D}^{2}\right)$.

$p=6$. This is similar to the $p=5$ case. In the extremal case $\left(r_{0}=0\right)$, the radius of the circular orbit is $r_{*}=\left(\ell_{T}^{2}+\ell \sqrt{\ell_{T}^{2}+L^{2}}\right) / L$ and it is stable. Similarly

$$
c_{\text {eff }}^{2}=\frac{1}{1+\ell_{N D}^{2} \sqrt{H_{6}}+\frac{\ell_{T}^{2}}{r_{*}^{2} \sqrt{H_{6}}}}
$$

This situation generalizes to $r_{0}>0$.

\subsubsection{The Hyper-universe}

We will advocate here a picture for the universe that is motivated by our previous discussion. It is the Copernican approach to the brane-universe idea and contains two levels.

1) Our conventional 4-dimensional universe is a p-brane embedded in a Ddimensional space. One possibility is $p=3$. However, we may have $p>3$ with the extra $p-3$ dimensions compactified. D maybe ten or eleven if the underlying theory is assumed to be string (M)-theory ${ }^{10}$.

2) The hyper-universe is populated by many brane-worlds in the same way that our universe is populated by stars. Brane-worlds gravitate (and in general interact via other related interactions) and can form "brane-galaxies" whose long range fields are those of "black-branes"

There are several fundamental questions/problems associated with such a picture.

(i) "Who ordered that?" It seems natural that if the fundamental theory contains brane-like objects then it is expected that the vacuum will be populated by them. The simplest examples of branes have to be infinite in size. We understand however contexts where compact branes can be stabilized by external fields 272] or a compact background geometry. Whether the energy of the hyper-universe is finite or not will depend on the type of branes (compact or not), the type of extra -dimensions (compact or not) and the potential presence of "negative tension branes".

Such branes appear in string theory (known as orientifold or orbifold planes) and are crucial for generating flat vacua in string theory. They cannot fluctuate,

\footnotetext{
${ }^{10} \mathrm{~A}$ reduced version of a universe containing branes has been advocated in 42 . The full Ddimensional space is assumed to be compact and the wrapped branes are treated like a gas in a finite box
} 
in order to preserve unitarity (their potential fluctuations have necessarily negative norm). They can "effectively move" via a changing background geometry. Their number in simple ground-states of string theory seems to be bounded above $\left(2^{5}\right.$ in the superstring and $2^{13}$ in the bosonic string, constrained by the tadpole of the Klein-bottle projection). Up to now, no fundamental reason is known for this fact. It is known however, that when their charge is large, they develop an "enhanconlike" singularity. Whether such a behavior is forbidding is not known. It seems however, than in compactifications with fluxes such upper bounds can be quite high. Macroscopic negative tension defects can provide interesting cosmological models [43]- 45].

(ii) There is no known reason so far, that a special dimensionality of branes is preferred. Thus, one may assume that originally any possible dimensionality of branes is produced. After a long evolution and process (that remains to be understood) the hyper-universe ends-up with a collection of hyper-galaxies, (gravitating collections of brane-worlds). Such aggregations with different dimension branes and arbitrary orientations of their world volumes run counter to the (strongly favored by data) homogeneity and isotropy of our own brane world.

A dynamical mechanism [46, 47, 48] can be argued to provide alignment of worldvolumes. However, a mechanism for the uniformisation of world-volume dimension needs to be found.

(iii) An important question is whether six (or D-4) extra dimensions are compact or (effectively) non-compact. An important experimental ingredient is that gravity at distances $10^{-5} m<l<10^{26} m$ has been measured to be of the $r^{-2}$ type (4dimensional).

(iiia) If the extra space-time dimensions are compact, there should be no phenomenological problems provided the compactness scale is small enough. If however the branes carry gauge charges (as D-branes do) the total charge of the branes is constrained to be zero. Thus, a hyper-universe requires an equal number of branes and anti-branes and orbifold/orientifold planes. The presence of anti-branes seems to be an important ingredient of some cosmological approaches to the early universe [49, 50, 63, 46, 47]. However, the branes might not have gauge charges but are stable because of dynamical reasons.

(iiib) An interesting alternative is that (some of) the extra dimensions are noncompact. A mechanism is needed to guarantee that gravity on our brane universe is four-dimensional in the required range of distances. There are three such mechanisms known as explained earlier:

(1) The internal space is non-compact with its Laplacian having a sufficiently large gap above zero [273]-275].

(2) The internal space is non-compact with a gap-less Laplacian with an appropriate density of eigenvalues close to zero, and finite volume [37]. It has been argued 
that this can be implemented in string theory [276]. In the case of codimension higher than one, an effective UV cutoff is needed in AdS. This maybe provided by angular momentum in the internal dimensions.

Both of the approaches (1) and (2) guarantee four dimensional gravity in the IR. In case (2) gravity becomes higher dimensional in the UV [173, 172].

(3) Quantum corrections of matter fields induce a localized Einstein term on the brane 51-55]. This term induces 4-dimensional gravity on the brane in the UV while gravity is higher dimensional in the IR. Obviously the cross-over scale has to be larger than the observable size of the universe or it has to be combined with one of the mechanisms described above 185, 172. Some model building in string theory has been done along these lines [277, 278, 183, 181, 180].

(iv) The cosmological evolution on our brane world is driven by matter localized on the brane and also bulk fields ("mirage cosmology" 41]). Such a picture, allows for novel cosmological mechanisms, namely inflow or outflow of matter from the bulk space [172], [56]- 59], that can trigger undesirable (constraining) effects [279], but can also provide natural mechanisms for inflation or late-time acceleration [56]-[59].

\subsection{Brane/antibrane systems and inflation}

Inflation is by now a mechanism favored for explaining the recent cosmological data as well as providing conceptual solutions to the flatness, horizon, and defects problems of cosmology. Although there many phenomenological models that implement inflation, there are two important problems remaining to be solved.

- The fine-tuning problem: Fine tuning is required in the phenomenological scalar models in order (i) to produce inflation (slow roll) (ii) to produce the experimentally observable size of CMB fluctuations 235, 280.

- The phenomenological model must be incorporated in the theory of fundamental interactions.

Here, we will describe the framework of brane-world inflation [281], and in partic-

ular, the one generated by the interaction of branes with anti-branes [46, 47, 50, 63]. There are also attempts to generate inflation for tachyon condensation [282- 301] but we will not discuss it here.

We will consider a pair of a $D 3 / \bar{D} 3$ brane and their static interaction potential

$$
V_{D \bar{D}}(r)=2 T_{3}\left(1-\frac{1}{2 \pi^{3}} \frac{T_{3}}{M_{10}^{8} r^{4}}\right)
$$

where $T_{3}$ is the tension of the branes, $M_{10}$ the ten-dimensional Planck scale, and $\mathrm{r}$ is the distance between the brane and anti-brane. The kinetic term of $r$ induced on the branes (in the low-velocity regime) is multiplied by $T_{3}$. Thus, the canonically normalized scalar is $\phi=\sqrt{T_{3}} r$ and the potential becomes

$$
V_{D \bar{D}}(\phi)=2 T_{3}\left(1-\frac{1}{2 \pi^{3}} \frac{T_{3}^{3}}{M_{10}^{8} \phi^{4}}\right)
$$


Such potentials can in principle give rise to inflation for large $\phi$. To investigate this we must investigate the slow-roll parameters [235, 280]

$$
\epsilon=\frac{M_{P}^{2}}{2}\left(\frac{V^{\prime}}{V}\right)^{2}, \quad \eta=M_{P}^{2} \frac{V^{\prime \prime}}{V}
$$

To get sufficient inflation we must arrange that $\epsilon<<1, \eta<<1$. Taking into account that if six dimensions transverse to the 3-branes are compact $M_{P}^{2}=M_{10}^{8} V_{6}$ where $V_{6}$ is the volume of the compact manifold we obtain

$$
\eta \sim \frac{V_{6}}{r^{6}}
$$

In order for the interaction potential to be valid, we must take $r<<V_{6}^{1 / 6}$. In that case $\eta>>1$ and inflation is not possible [46, 47]. This result is valid also in the anisotropic case [46, 47, 308].

Things can get better if we consider a warped background geometry. This is equivalent to consider a large number of $D 3$ branes, which in this case gravitate. In the near horizon limit they generate (locally) an $A d S_{5}$ geometry. Of course, this is part of the compact manifold, so the $A d S_{5}$ must be cutoff at some point. The metric of $A d S_{5}$ in Poincaré coordinates is

$$
d s^{2}=\frac{r^{2}}{R^{2}}\left(-d t^{2}+d \vec{x}^{2}\right)+\frac{R^{2}}{R^{2}} d r^{2}
$$

and there is the standard 4-form background. The AdS length-scale is given by [20]

$$
R^{4} \sim g_{s} N \alpha^{\prime 2}
$$

The exact constant of proportionality depends on the embedding of the AdS slice in the six-dimensional compact manifold.

We will the assume that the range of $r$ is $r_{0}<r<r_{\max }$ for $r>r_{\max }$ (the UV region) the manifold is glued to the internal compact manifold. The manifold is also cut-off in the IR $\left(r<r_{0}\right)$ smoothly as in solutions dual to confining gauge theories (see for example [356]) Thus, this region is approximate to the simple RS setup with an UV and an IR brane [36]. In particular, there is an effective four-dimensional gravitational interaction, with a finite Planck mass due to the UV cutoff $r_{\max }$.

We would like now to consider the effective world-volume action of a $D_{3}$ brane in this background. The $C_{4}$ form is the near horizon limit of (7.5.1.3)

$$
C_{4}=\frac{r^{4}}{R^{4}}
$$

and the induced action reads

$$
S_{D_{3}}=-T_{3} \int d^{4} x \sqrt{-g} \frac{r^{4}}{R^{4}} \sqrt{1-\frac{R^{4}}{r^{4}} g^{\mu \nu} \partial_{\mu} r \partial_{\nu} r}+T_{3} \int d^{4} x \frac{r^{4}}{R^{4}}
$$


For a $\bar{D}_{3}$, the sign of the second term is reversed.

For a $D_{3}$ brane in this background the static potential cancels (no force condition, for a BPS configuration). However, in the presence of $D_{3}$ brane at $\mathrm{r}$ and a $\bar{D}_{3}$ at $r_{0}$ there is a non-trivial potential that can be estimated as follows: The harmonic function in the metric due to $\mathrm{N}_{3}$ branes is

$$
h(r)=\frac{R^{4}}{r^{4}}
$$

The analogous function for an extra $D_{3}$ brane at $r=r_{*}$ is

$$
h(r)+\delta h(r)=R^{4}\left[\frac{1}{r^{4}}+\frac{1}{N} \frac{1}{r_{*}^{4}}\right]
$$

Thus, the potential for a $\bar{D}_{3}$ at $r=r_{0}$ and a $D_{3}$ brane at $\mathrm{r}$, for large $\mathrm{r}$, from the DBI action is

$$
V=2 T_{3} \frac{r_{0}^{4}}{R^{4}}\left[1-\frac{1}{N} \frac{r_{0}^{4}}{r^{4}}\right]
$$

There are two observations on this interaction. Th first term is there but does not affect the motion of the $D_{3}$ brane. the second term is responsible for the attractive interaction between the brane and anti-brane. At large distance, this is a slowly varying potential with an "effective" tension

$$
T_{e f f}=T_{3} \frac{r_{0}^{4}}{R^{4}}<<T_{3}
$$

Thus in this regime we expect that

$$
S_{D_{3} \bar{D}_{3}}=\int d^{4} x\left[\frac{T_{3}}{2} g^{\mu \nu} \partial_{\mu} r \partial_{\nu} r-2 T_{3} \frac{r_{0}^{4}}{R^{4}}\left(1-\frac{1}{N} \frac{r_{0}^{4}}{r^{4}}\right)\right]
$$

will give rise to slow roll and inflation. When eventually the branes collide, there will be brane-anti-brane annihilation. This could be the starting point of reheating. There are, however, several problems with this type of inflationary scenarios 308 . They have to do with additional couplings on the D-branes that might destroy inflations. There is active research currently on this realization of inflation, 309]-324, but it seems that the last word has not yet been said.

\subsection{The cosmology of massive gravity and late time acceleration}

It has been argued in 7.3.7 that sometimes four-dimensional gravity in the UV can be mediated by a massive-like graviton. It turns out that in all case where branes and induced gravity are involved, four-dimensional gravitons are massive in some regime. We will investigate here the cosmological evolution of a universe where gravity is massive. We will show that a very light graviton produces a late time acceleration of the universe that has the right properties to explain today's data. 
The cosmological equations have been derived in 325 for quadratic potential and in [326, 327] for more general potentials. For simplicity I will follow the treatment in [325], although some of the conclusions will turn out to be different.

We will consider a four-dimensional massive graviton field $h_{\mu \nu}$ in a background Minkowski metric $\eta_{\mu \nu} \sim(1,-1,-1,-1)$. The case of a general background constant metric is equivalent to the above, upon appropriate constant rescalings of the scale factors in $g_{\mu \nu}$. The full metric is defined as

$$
\sqrt{-g} g^{\mu \nu}=\sqrt{-\eta}\left(\eta^{\mu \nu}+h^{\mu \nu}\right)
$$

and $g_{\mu \nu}$ is the inverse of $g^{\mu \nu}$. Matter couples to gravity via a minimal coupling to $g_{\mu \nu}$

The action is given by

$$
L=L_{G R}+L_{\text {mass }}+L_{\text {matter }}
$$

where

$$
L_{G R}=-\frac{1}{2 \kappa^{2}} \sqrt{-g} R \quad, \quad L_{m a s s}=-\frac{1}{2 \kappa^{2}} \sqrt{-\eta}\left[k_{1} h^{\mu \nu} h_{\mu \nu}+k_{2}\left(h^{\mu \nu} \eta_{\mu \nu}\right)^{2}\right]
$$

Here we have added only a quadratic mass term. We will comment on a more general potential later.

A study of the quadratic fluctuations of the graviton field indicates the presence of a massive spin-two filed with mass $m_{g}$ and a scalar component with mass $m_{0}$. The masses are related to $k_{1,2}$ as [325]

$$
k_{1}=\frac{m_{g}^{2}}{4} \quad, \quad k_{2}=-\frac{m_{g}^{2}}{8} \frac{m_{g}^{2}+2 m_{0}^{2}}{2 m_{g}^{2}+m_{0}^{2}}
$$

We define for further convenience

$$
\zeta=\frac{m_{0}^{2}}{m_{g}^{2}}
$$

The field equations obtained are

$$
G_{\mu \nu}+M_{\mu \nu}=T_{\mu \nu} \quad, \quad G_{\mu \nu}=R_{\mu \nu}-\frac{1}{2} g_{\mu \nu} R
$$

where

$$
M_{\mu \nu}=\left(\delta_{\mu}^{a} \delta_{\nu}^{b}-\frac{1}{2} g^{a b} g_{\mu \nu}\right)\left(2 k_{1} h_{a b}+2 k_{2}\left(h^{c d} \eta_{c d}\right) \eta_{a b}\right)
$$

and $T_{\mu \nu}$ is the matter stress tensor.

The homogeneous and isotropic cosmological ansatz is

$$
h^{00}=\frac{a^{3}}{b}-1 \quad, \quad h^{11}=h^{22}=h^{33}=1-a b
$$


where $a, b$ are functions of time. Then, the components of the full metric, defined in (7.7.1), are

$$
g_{00}=b^{2} \quad, \quad g_{11}=g_{22}=g_{33}=-a^{2}
$$

We also assume a dilute homogeneous matter distribution

$$
T_{00}=\rho \quad, \quad T_{11}=T_{22}=T_{33}=-p=-w \rho
$$

which is conserved

$$
\dot{\rho}+3(1+w) \frac{\dot{a}}{a} \rho=0
$$

where the dot implies differentiation with respect to cosmological time $\tau$ defined as

$$
\frac{d}{d \tau}=\frac{1}{b} \frac{d}{d t}
$$

Substituting the cosmological ansatz (7.7.8) into the gravitational equations 7.7 .6$)$ we obtain

$$
3\left(\frac{\dot{a}}{a}\right)^{2}+M_{0}^{0}=\kappa^{2} \rho \quad, \quad 2 \frac{d}{d \tau}\left(\frac{\dot{a}}{a}\right)+3\left(\frac{\dot{a}}{a}\right)^{2}+M_{1}^{1}=-\kappa^{2} p
$$

where

$$
\begin{gathered}
M_{0}^{0}=\frac{3 m_{g}^{2}}{8(\zeta+2)} \frac{a^{5}+(4 \zeta-1) a b^{4}+2 \zeta a^{2} b-6 \zeta b^{3}}{a^{2} b^{3}} \\
M_{1}^{1}=-\frac{m_{g}^{2}}{8(\zeta+2)} \frac{3 a^{5}-4(1+2 \zeta) a^{3} b^{2}+(1-4 \zeta) a b^{4}+6 \zeta a^{2} b+6 \zeta b^{3}}{a^{2} b^{3}}
\end{gathered}
$$

The integrability condition on $M$ is

$$
\dot{M}_{0}^{0}+3\left(\frac{\dot{a}}{a}\right)\left[M_{0}^{0}-M_{1}^{1}\right]=0
$$

Thus, the first of the equations in (7.7.13) and (7.7.16) are an equivalent set of equations, from which the second equation in (7.7.13) follows.

Equation (7.7.16) can be integrated explicitly to the following algebraic equation

$$
3 \frac{a^{6}}{b^{2}}+(4 \zeta-1) a^{2} b^{2}-2(2 \zeta+1) a^{4}+8 \zeta \frac{a^{3}}{b}-8 \zeta=0
$$

We have chosen the constant of integration such that Minkowski space $(a=b=1)$ is a solution.

In the phenomenologically interesting case, $m_{0}>m_{g}$ so that $\zeta \rightarrow \infty$. (in fact $\left.\zeta \sim 10^{30}\right)$. The $\zeta \rightarrow \infty$ limit corresponds to $k_{2}=-k_{1}$ and the mass term becomes of the Pauli-Fierz type. As it was shown in 328] in this case the validity of the effective action is enhanced up to scales $\sim\left(M_{P} m_{g}^{4}\right)^{\frac{1}{5}}$. 
The algebraic condition (7.7.17) in the $\zeta \rightarrow \infty$ limit becomes

$$
a^{2} b^{3}-\left(a^{4}+2\right) b+2 a^{3}=0
$$

while the Friedman equation is

$$
\left(\frac{\dot{a}}{a}\right)^{2}=\frac{\kappa}{3} \rho+\rho_{m} \equiv \frac{\kappa}{3} \rho-\frac{m_{g}^{2}}{4}\left(2 \frac{b}{a}+\frac{1}{b^{2}}-3 \frac{1}{a^{2}}\right)
$$

The algebraic equation (7.7.18) can be solved exactly for $\mathrm{b}$ as a function of a, which can then be substituted in (7.7.19) to give an equation for $a(b)$. However, we are interested in the large $a$ behavior of the evolution. For $a>>1$ the three solutions of (7.7.18) are

$$
b_{ \pm}= \pm a-\frac{1}{a} \mp \frac{1}{2 a^{3}}+\cdots \quad, \quad b_{3}=\frac{2}{a}+\frac{4}{a^{5}}+\cdots
$$

while the effective energy density due to the massive graviton becomes

$$
\left.\frac{\rho_{m}}{m_{g}^{2}}\right|_{ \pm}=\mp \frac{1}{2}+\frac{1}{a^{2}} \mp \frac{1}{4 a^{4}}+\cdots \quad,\left.\quad \frac{\rho_{m}}{m_{g}^{2}}\right|_{3}=-\frac{a^{2}}{16}-\frac{1}{4 a^{6}}+\cdots
$$

Out of the three solutions, only the minus one is real for all values of a. The other two are complex when $1<a<(1+\sqrt{3})^{\frac{3}{4}}$. Moreover, $\rho_{m}$ is a monotonically decreasing positive function of $a$.

Thus, the physical solution is the minus one, and as can be seen from (7.7.21) it gives a positive effective cosmological constant at late times, $\Lambda_{\mathrm{eff}}=\frac{m_{g}^{2}}{2}$. It should also be remarked that the subleading contribution to the energy density behaves as an effective curvature term with $k_{\text {eff }}=-1$.

It is intriguing that the value of the graviton mass needed to be consistent with standard massless gravity inside today's horizon, $m_{g} \sim H_{\text {today }}^{-1}$ gives a contribution to the vacuum energy which is of the same order of magnitude as that coming from cosmological observations.

As it was argued in 328 massive gravity with a Fierz-Pauli term becomes strongly coupled at energies of the order $E_{5} \sim\left(m_{g}^{4} M_{P}\right)^{1 / 5}$. Adding higher potential terms in $h$ and fine-tuning such interactions can increase this cutoff to $E_{3} \sim$ $\left(m_{g}^{2} M_{P}\right)^{1 / 3}$. It is conceivable that the inclusion of interactions with other fields (namely the dilaton and axion) may increase this cutoff even further to $E_{2} \sim$ $\left(m_{g} M_{P}\right)^{1 / 2}$. To obtain some numbers we set the graviton mass to the inverse horizon size today $m_{g} \sim\left(10^{26} \mathrm{~m}\right)^{-1}$ and we obtain

$$
E_{5}^{-1} \sim 10^{19} \mathrm{~m} \quad, \quad E_{3}^{-1} \sim 10^{6} \mathrm{~m} \quad, \quad E_{2}^{-1} \sim 10^{-4} \mathrm{~m}
$$

It is interesting that a massive gravity with a $E_{2}$ cutoff breaks down precisely at the boundary of today's short distance experimental tests. The lower cutoffs indicate that such theories are not viable for describing four-dimensional gravity. 


\subsubsection{Cubic interactions}

In general there will be higher order terms in the potential of the massive graviton. To see how such terms affect the late cosmological evolution we will study the cubic terms.

The most general cubic interaction is

$$
L_{3}=-\frac{1}{2 \kappa} \sqrt{-\eta}\left(r_{1}\left(h^{\mu}{ }_{\mu}\right)^{3}+r_{2}\left(h^{\mu}{ }_{\mu}\right) h^{\nu \rho} h_{\nu \rho}+r_{3} h^{\mu \nu} h_{\mu \rho} h^{\rho}{ }_{\nu}\right)
$$

where $r_{i}$ have dimensions of (mass) ${ }^{2}$.

The correction to the energy density is

$$
\begin{gathered}
\rho_{3}=-\frac{r_{1}}{2} \frac{\left(a^{2}-3 b^{2}\right)\left(a^{3}-4 b+3 a b^{2}\right)^{2}}{a^{2} b^{4}}- \\
-\frac{r_{2}}{2} \frac{a^{8}-4 a^{5} b+4 a^{2} b^{2}+a^{6} b^{2}-12 b^{4}-a^{4} b^{4}+20 a b^{5}-9 a^{2} b^{6}}{a^{2} b^{4}}- \\
-\frac{r_{3}}{2} \frac{a^{8}-2 a^{5} b+a^{2} b^{2}-3 b^{4}+6 a b^{5}-3 a^{2} b^{6}}{a^{2} b^{4}}
\end{gathered}
$$

The algebraic condition, generalizing (7.7.17) is

$$
I_{2}+I_{3}=0
$$

where

$$
\begin{gathered}
b^{2} I_{2}=3\left(k_{1}+k_{2}\right) a^{6}+4\left(k_{1}+4 k_{2}\right)\left(b^{2}-a^{3} b\right)+6 k_{2} a^{4} b^{2}-3\left(k_{1}+3 k_{2}\right) a^{2} b^{4} \\
b^{3} I_{3}=-6\left(r_{3}+3 r_{2}+9 r_{1}\right) a^{3} b^{6}+3\left(3 r_{3}+10 r_{2}+36 r_{1}\right) a^{2} b^{5}-4\left(r_{3}+4 r_{2}+16 r_{1}+3\left(r_{2}+6 r_{1}\right) a^{4}\right) b^{3}+ \\
+6\left(r_{3}+4 r_{2}+16 r_{1}+\left(r_{2}+3 r_{1}\right) a^{4}\right) a^{3} b^{2}-9\left(r_{3}+2 r_{2}+4 r_{1}\right) a^{6} b+4\left(r_{1}+r_{2}+r_{3}\right) a^{9}
\end{gathered}
$$

The asymptotic form of the energy density after solving for $b$ is of the form

$$
\rho=m_{1}^{2}+m_{2}^{2} a^{2}+\mathcal{O}\left(\frac{1}{a^{2}}\right)
$$

where the masses $m_{1,2}$ are functions of $k_{1,2}, r_{1,2,3}$. This gives again eternal acceleration. In particular, $m_{2}=0$ when $r_{3}+4 r_{2}+16 r_{1}=0$ (and $m_{1}^{2}=-2 k_{1}-5 k_{2}$ as without the cubic terms) or when $r_{2}=-\frac{3}{7} r_{1}, r_{3}=-\frac{4}{7} r_{1}$ (and $m_{1}^{2}=\frac{14 k_{1}-35 k_{2}-780 r_{1}}{7 \sqrt{15}}$ ).

At late times, ignoring the subleading terms, the effective energy density (7.7.1.6) provides super-acceleration for $m_{2}^{2}>0$

$$
a(t)=2 \frac{m_{1}}{m_{2}} \frac{C e^{m_{1} t}}{1-C^{2} e^{2 m_{1} t}}
$$

For $m_{2}^{2}<0$, we have initially acceleration and later exponential deceleration.

Thus, higher order terms in the potential do not substantially affect the nature of late cosmology (although they may affect the details). 


\subsection{Massive gravity and gauge theory}

In this section we will present yet another idea on the realization of 4-d gravity. There are several motivations for the approach advocated here:

- Closed string theory generically predicts gravity. Fundamental string theories provide a consistent (perturbative) quantization of gravity. Despite its successes, string theory, although well defined at energies below or at the string scale, breaks down at energies close to the Planck scale. In particular, the perturbation theory breaks down due to the strong effective gravitational coupling. Despite speculations, the nature of the extreme UV degrees of freedom of the theory is still obscure [329][334]. Perturbative string theory is essentially a cutoff theory of gravity and other interactions. This is obvious at the one-loop level of closed string theory, where the theory has a (smart indeed) cutoff at the string scale, implemented by Schwinger parameters confined to the fundamental domain of the torus. A similar structure persists at higher orders in perturbation theory. In perturbative string theory, the string scale is much lower than the Planck scale. Taking this at face value, we do not expect the theory to give useful information about physics at energies hierarchically higher than the string scale, namely around or above the Planck scale without running at a singularity/strong coupling.

It would seem that non-perturbative dualities might give a way out, since they provide information about strong coupling physics. Indeed non-perturbative dualities relate theories with different (dimensionless) couplings and string scales. This is however not the case for gravity, since any non-perturbative duality we know, leaves the Planck scale fixed, and thus cannot address questions on physics at or beyond the Planck scale.

- Since the early work of 't Hooft [336] it was understood that the low energy limit of large N-gauge theories is described by some string theory. The gauge theory versus string theory/gravity correspondence [20, 21] is a more precise indication that gravity can be realized as an effective theory of a four-dimensional gauge theory. The inverse is also true: fundamental string theory in some backgrounds describes the physics of theories that at low energy are standard gauge theories. Although bulkboundary duality is a concept transcending that of four-dimensional gauge theories, it is most powerful in the four-dimensional cases.

We can claim that the lesson of AdS/CFT correspondence is that any gauge theory has a dual gravity/string theory. The idea of 't Hooft that gravity must be holographic [337, 338] indicates that a gravity theory must have a dual gauge theory description.

- A standard gauge theory realization of four-dimensional gravity generically predicts massive composite gravitons. The graviton is the spin-two glueball generated out of the vacuum by the stress-tensor of the theory. Confinement typically comes together with a mass gap. A graviton mass is severely constrained by observations. 
Its presence may have two potential advantages. It predicts an intrinsic cosmological constant that may be of the order of magnitude observed today, if the graviton mass modifies gravity at or beyond the horizon today. Also, the fact that the graviton is a bound state, provides mechanism for suppressing the cosmological constant. In particular, the graviton does not directly couple to the standard "vacuum energy" of the SM fields.

Thus, the idea is that the building blocks of a theory of all interactions are four-dimensional gauge theories. Such theories are special in many respects both nature-wise and mathematics-wise. Four-dimensional gravity is an effective, almost classical theory, emanating from a large- $\mathrm{N}$ sector of the gauge theory.

The approach advocated here has similarities with ideas in [340, 341] and 342. The qualitative model of 340, is somewhat different since the SM particles are not charged under the strong gauge group. Gravity here is mediated by (heavy) messager matter charged both under the SM group and the strong gauge group, as suggested by gauge-theory/string theory correspondence. In fact, a light scalar graviton can be a meson but not a spin-two one. There is also some similarity with the idea of deconstruction 343, but here it is gravity rather than higher dimensional matter theories that is realized by the gauge theory.

There are direct similarities with attempts to describe fundamental string theory in terms of matrix models 344-348. Here, however, the gauge physics is four dimensional and provides a wider class of gravity theories. Moreover, a four-dimensional large $\mathrm{N}$ gauge theory, although more complicated than a standard Matrix model gives a better intuitive handle on the physics.

Consider a large N-gauge theory with gauge group $G_{N}$ and large-N matter (scalars and fermions) that we will not specify at the moment. We would like the theory to be asymptotically free or conformal, so that it is a well-defined theory at all scales. This will put constraints on the type of large- $\mathrm{N}$ matter content.

At low energy, the effective degrees of freedom are colorless glueballs as well as mesons (baryons are heavy at large N, [335]). Among the effective low-energy degrees of freedom there is always a spin-two particle (that is generated from the gauge theory vacuum by the total stress tensor of theory). Typically this theory, being confining, will have a mass gap, and the spin-two particle will be massive. However, on general principles (conservation of the gauge theory stress-tensor) we expect to have a spin-two gauge invariance (that may be spontaneously broken by the gauge theory vacuum). Thus, the interactions of this particle, are those of a massive graviton.

There are, however, other universal composites. Let us consider for simplicity an $S U(N)$ pure gauge theory. The leading operators, that are expected to create glueballs out of the vacuum are a scalar (the "dilaton") $\phi \rightarrow \operatorname{Tr}\left[F_{\mu \nu} F^{\mu \nu}\right]$, a spin-2 "graviton" $g_{\mu \nu} \rightarrow \operatorname{tr}\left[F_{\mu \rho} F^{\rho}{ }_{\nu}-\frac{1}{4} \delta_{\mu \nu} F_{\rho \sigma} F^{\rho \sigma}\right]$ and a pseudoscalar "axion" $a \rightarrow \epsilon^{\mu r \sigma} \operatorname{Tr}\left[F_{\mu \nu} F_{\rho \sigma}\right]$ [339]. These particles will be massive, and their interactions at low energy are non- 
perturbative from the point of view of the gauge theory. In this relatively simple theory, with two parameters, the mass scale $\Lambda$ and $N$, the masses are expected to be of order $\Lambda$ and the interactions controlled at large $\mathrm{N}$ by $g_{s} \sim \frac{1}{\sqrt{N}}$. In particular, three point couplings scale as $1 / \sqrt{N}$.

As first advocated by 't Hooft [336], the effective interactions of the glueballs are expected to be described by an effective string theory in the low energy regime. Most probably, the world-sheets of this string theory are discrete, and only a tuning of the gauge theory (double scaling limit?) might give rise to a continuous string.

If the large- $\mathrm{N}$ gauge theory contains fermions in the fundamental, $\psi_{a}^{i}$ ( $i$ is a flavor index while $a$ is color), then there are other generic low energy bound states. In particular, the conserved fermionic currents $J_{\mu}^{a} \sim \bar{\psi}_{a}^{i} T_{i j} \gamma^{\mu} \psi_{a}^{j}$ generate (generically massive) vector particles. The scalar or pseudoscalar densities $\bar{\psi}_{a}^{i} \psi_{a}^{j}, \bar{\psi}_{a}^{i} \gamma^{5} \psi_{a}^{j}$ correspond to scalar or pseudoscalar mesons, (and give rise to open strings). The antisymmetric tensor composites $\bar{\psi}_{a}^{i} \gamma^{\mu \nu} \psi_{a}^{j}, \bar{\psi}_{a}^{i} \gamma^{\mu \nu} \gamma^{5} \psi_{a}^{j}$ should correspond to higher oscillator string states, and must consequently be heavier.

Finally, adjoint scalars give rise to extra scalar bound-states that appear as extra internal dimensions, as AdS/CFT indicates.

Unlike fundamental string theory, the graviton here is a bound state of glue, and in the UV, the proper description of its interactions are in terms of gluons. Thus, in this theory, the low energy theory is string-like. But the hard scattering of "gravitons" is described by perturbative gauge theory, while their soft scattering by an effective (massive) gravity/string-theory. In particular, gravitational interactions turn off at high energy due to asymptotic freedom.

There are several immediate questions that beg to be answered in such a scheme.

(A) The effective graviton must have a mass that is very small (probably of the order of the inverse horizon size) in order not to be upset by current data. Just lowering the scale $\Lambda$ of the gauge theory is not enough. A simple gauge theory with an ultra-low $\Lambda$ (of the order of the inverse horizon size today), has light gravitons that are on the other hand very loosely bound states with a size comparable to that of the universe. We need that their size is hierarchically larger than their mass. An important issue is whether a small mass for the graviton is technically natural. It is conceivable that coordinate invariance protects the graviton mass as gauge invariance does for the photon mass.

Moreover, the other generic low lying scalars (dilaton and axion as well as the spin-0 component of the graviton) must be substantially heavier so that we are not again upset by data.

What types of large N-gauge theories have a small or no mass gap? What determines the mass gap? What determines the hierarchy of masses of $\phi, g_{\mu \nu}$ and $a$ ? Although, there has been considerable efforts to answer such questions for several 
gauge theories, no unifying picture exists ${ }^{11}$. This is due to the fact that these questions involve non-perturbative gauge theory physics. It is also due to the fact that glueballs have been conspicuously absent from particle physics experiments. Such questions may be studied using the general ideas of AdS/CFT correspondence and its generalizations.

An important lesson from AdS/CFT correspondence is that the gravity dual to four-dimensional gauge theory is five-dimensional (with additional compact dimensions if extra (adjoint) scalar matter appears in the gauge theory). Polyakov has advocated general reasons why this is expected [350]. Indeed counting the degrees of freedom of massive $g_{\mu \nu}, \phi, a$ we could expect that their effective interaction can be described by a five-dimensional massless graviton as well as five-dimensional scalars $\phi$ and $a$. The non-trivial gauge theory vacuum should correspond to a nontrivial background of the five-dimensional theory (as $A d S_{5} \times S^{5}$ describes $\mathrm{N}=4$ super Yang-Mills via AdS/CFT duality).

We would also like the full theory to be asymptotically free. In that case the short distance physics will be well defined. In the five-dimensional picture this will imply an $A d S_{5}$ asymptotic region.

(B) At low energy in the gauge theory (if it is confining), the effective physics is described by some string theory (at large N). Also non-confining theories have a string description as AdS/CFT indicates but only for the gauge singlet sector. The important question is: what are the scales of the string theory/gravity in terms of the fundamental scales of the gauge theory? The AdS/CFT paradigm is suggestive. Here, on the string theory side there are three parameters: The AdS radius $R$, the string scale $l_{s}$ and the string coupling $1 / N$. On the gauge theory side there are only two: $\mathrm{N}$ and the 't Hooft coupling $\lambda=g^{2} N . \mathrm{N}=4$ super Yang-Mills is scale invariant in the symmetric vacuum. This implies that only the ratio $R / l_{s}$ is observable: $R / l_{s}=\lambda^{1 / 4}$.

When a mass gap $\Lambda$ is generated because of temperature effects, it corresponds to a long distance (far away from boundary) cutoff in AdS, namely the position $r_{0}$ of the horizon of the AdS black-hole, $r_{0}=\Lambda R^{2}$. The energy on the AdS side is given by $E=r / R^{2}$. The cutoff implies a non-trivial effective string length for the gauge theory, obtained by red-shifting the AdS string scale at the horizon [351]:

$$
l_{s}^{\mathrm{eff}} \sim l_{s} \frac{r_{0}}{R} \sim \frac{1}{\lambda^{1 / 4} \mathrm{E}}
$$

Finally there could be masses and/or Yukawa couplings in the large- $\mathrm{N}$ gauge theory. They modify the higher-dimensional geometry by turning on fluxes [356, 357]

\footnotetext{
${ }^{11}$ For example, in $\mathrm{SU}(2)$ and $\mathrm{SU}(3)$ gauge theory there is an inverted hierarchy for the $0^{++}$and $2^{++}$glueballs, 349.
} 
(C) Another important question is: how is the SM accommodated in such a picture? The expectation is that the SM gauge group is a separate factor from the large-N group. It may be so by fiat, or it may be connected to the large-N gauge group $G_{N}$ by symmetry breaking. It could also be enlarged to a unified group (SU(5), SO(10) etc). The standard model particles are neutral under $G_{N}$. There should be new massive particles charged under both the $G_{N}$ and the SM gauge group. Integrating out these particles, the gravitational interaction is generated for the standard model particles. Thus, such particles are messagers of the gravitational interaction. This is analogous to the picture we have of probe D-branes in AdS/CFT [352]-[355, 234, 270]

One could also advocate a certain "unification" in this context: The theory starts from a simple large- $\mathrm{N}$ gauge group which is broken to a large-N subgroup generating gravity, as well as "splinters" (the SM or the conventional unified group). The massive states communicate gravity to the SM particles.

(D) The issue of the cosmological constant is qualitatively different here. The standard matter loop diagrams that contribute to the cosmological constant do not couple to gravity here. Matter loops induce a potential for the graviton. Since the graviton is composite, its form factors cut-off the matter contributions at much lower energies (hopefully at $10^{-3} \mathrm{eV}$ ) than the matter theory cutoff. This is similar to the mechanism advocated in [340.

(E) As we have learned from AdS/CFT, and expected on more general principles [350] the low energy gravitational theory of a large $\mathrm{N}$-gauge theory have at least five non-compact dimensions. The obvious question is: how is this compatible with the observed 4-d gravity. Here there are two complementary ideas that might resolve this puzzle: RS localization and brane induced gravity. The RS solution can be implemented if the "vacuum" of the gauge theory imposes an effective UV cutoff at the position of the SM branes. Brane induced gravity is always present, however its strength is crucial for it to effectively turn gravity four-dimensional in agreement with data.

(F) Some of these questions can be put in perspective by utilizing the essentials of the D-brane picture which underlies gauge-theory/gravity correspondence. They provide a close link between gauge theory and gravity.

The large $\mathrm{N}$ gauge group $G_{N}$ can be represented as a heavy (large N) black brane. The SM gauge group can be viewed as some collection of a few probe branes in the background of the black-hole. Ideally, integrating out the strings that connect the probe branes with the central stack (massive matter charged under both $G_{N}$ and $\mathrm{SM}$ ) induce effective gravitational interactions for the SM fields. If more than five 
dimensions are present, the SM branes may be in a nearly stable orbit around the central black-hole. Thus, the picture with a single large-N component of the gauge group gives a hyper-planetary model. The SM branes must be very close to the central black hole (near horizon region). Falling on the naked (or not) singularity is catastrophic since it implies fusion of the SM gauge group with $G_{N}$ and a radical rearrangement of the IR field theory (gauge group enhancement) . There could be other simple components of the gauge group, giving presumably rise to other hyperplanetary systems and eventually to hyper-galaxies and a hyper-universe.

Here, the picture of the gauge theory representation for gravity we advocate, matches smoothly to the cosmological evolution of D-branes we discussed in previous sections.

(G) There is an extra issue of divergences. We usually assume that a 4-d large$\mathrm{N}$ gauge theory has $4 \mathrm{~d}$-type divergences. However this need not be true. As a counterexample, consider a 5-D theory, and compactify one direction on a lattice. This is a large- $\mathrm{N}$ 4-d theory, (where $\mathrm{N}$ is the number of lattice sites) but in the continuum (large N) limit should reproduce the 5-D divergencies for certain energies. Such divergencies will thus show up as large-N divergences, and understanding them is central in this context.

(H) The approach described here has a potentially serious problem: it relies on non-perturbative physics. Typically such a problem proves fatal. However here we would like to advocate a 5-dimensional gravitational approach to the problem. Several of the questions described above can be attacked in this fashion, namely determining the 5-d action and its vacuum solution and tuning it to achieve small graviton mass and correct gravitational interactions for standard model particles. In the next subsections we start a preliminary investigation of some simple issues in this context. Whether a fully workable model can emerge remains to be seen.

\section{Acknowledgements}

I would like to thank my collaborators, P. Anastasopoulos, I. Antoniadis, A. Kehagias, G. Kofinas, J. Rizos, N. Tetradis, T. Tomaras, V. Zarikas who have contributed to some of the physics presented here. I would like to thank S. Alexander, C. Bachas, T. Banks, R. Blumenhagen, R. Brandeberger, R. Brustein, C. Charmousis, J. Cline, L. Cornalba, M. Costa, A. Davis, N. Deruelle, G. Dvali, L. Ibañez, N. Kaloper, J. Khoury, C. Kounnas, D. Langlois, D. Lüst, D. Marolf, R. Myers, A. Petkou, M. Porrati, F. Quevedo, H. Real, C. Skenderis, D. Steer, S. Trivedi, A. Uranga C. Van 
der Bruck, G. Veneziano and A. Zaffaroni for discussions and correspondence and M. Graña, A. Hammou and H. Partouche for comments and a careful reading of the manuscript. This review is an expanded version of lectures given at the RTN school in Torino, the Ahrenshoop Symposium and the graduate school of Roma I University. I would like to thank the organizers for giving me the opportunity to give these lectures.

The author acknowledges partial support from RTN contracts HPRN-CT-200000122, -00131, -00148 and INTAS contract N 2000-254,. 


\section{References}

[1] D. J. Gross, J. A. Harvey, E. J. Martinec and R. Rohm, "The Heterotic String," Phys. Rev. Lett. 54 (1985) 502; Nucl. Phys. B 256 (1985) 253; Nucl. Phys. B 267 (1986) 75.

[2] I. Antoniadis, J. R. Ellis, J. S. Hagelin and D. V. Nanopoulos, "The Flipped SU(5) X U(1) String Model Revamped," Phys. Lett. B 231 (1989) 65.

[3] I. Antoniadis, G. K. Leontaris and J. Rizos, "A Three Generation SU(4) X O(4) String Model," Phys. Lett. B 245 (1990) 161.

[4] J. Scherk and J. H. Schwarz, "How To Get Masses From Extra Dimensions," Nucl. Phys. B 153 (1979) 61.

[5] S. Ferrara, C. Kounnas and M. Porrati, "Superstring Solutions With Spontaneously Broken Four-Dimensional Supersymmetry," Nucl. Phys. B 304 (1988) 500;

Phys. Lett. B 206 (1988) 25.

[6] C. Kounnas and M. Porrati, "Spontaneous Supersymmetry Breaking In String Theory," Nucl. Phys. B 310 (1988) 355.

[7] S. Ferrara, C. Kounnas, M. Porrati and F. Zwirner, "Superstrings With Spontaneously Broken Supersymmetry And Their Effective Theories,"

Nucl. Phys. B 318 (1989) 75

[8] E. Kiritsis and C. Kounnas, "Perturbative and non-perturbative partial supersymmetry breaking: $\mathrm{N}=4 \rightarrow \mathrm{N}=2 \rightarrow \mathrm{N}=1$," Nucl. Phys. B 503 (1997) 117; [arXiv:hep-th/9703059].

[9] L. J. Dixon, V. Kaplunovsky and C. Vafa, "On Four-Dimensional Gauge Theories From Type Ii Superstrings," Nucl. Phys. B 294 (1987) 43.

[10] A. Sagnotti, "Open Strings And Their Symmetry Groups," arXiv:hep-th/0208020

[11] G. Pradisi and A. Sagnotti, "Open String Orbifolds," Phys. Lett. B 216 (1989) 59.

[12] P. Horava, "Background Duality Of Open String Models," Nucl. Phys. B 327 (1989) 461; Phys. Lett. B 231 (1989) 251.

[13] M. Bianchi and A. Sagnotti, "On The Systematics Of Open String Theories," Phys. Lett. B 247 (1990) 517; "Twist Symmetry And Open String Wilson Lines," Nucl. Phys. B 361 (1991) 519.

[14] M. Bianchi, G. Pradisi and A. Sagnotti, "Toroidal compactification and symmetry breaking in open string theories," Nucl. Phys. B 376 (1992) 365.

[15] D. Fioravanti, G. Pradisi and A. Sagnotti, "Sewing constraints and nonorientable open strings," Phys. Lett. B 321 (1994) 349 [arXiv:hep-th/9311183]. 
[16] C. Angelantonj, M. Bianchi, G. Pradisi, A. Sagnotti and Y. S. Stanev, "Chiral asymmetry in four-dimensional open- string vacua," Phys. Lett. B 385 (1996) 96; [arXiv:hep-th/9606169].

[17] C. M. Hull and P. K. Townsend, "Unity of superstring dualities," Nucl. Phys. B 438 (1995) 109 [arXiv:hep-th/9410167].

[18] E. Witten, "String theory dynamics in various dimensions," Nucl. Phys. B 443 (1995) 85 [arXiv:hep-th/9503124]; [arXiv:hep-th/9507121].

[19] J. Polchinski, "Dirichlet-Branes and Ramond-Ramond Charges," Phys. Rev. Lett. 75 (1995) 4724 [arXiv:hep-th/9510017.

[20] J. M. Maldacena, "The large N limit of superconformal field theories and supergravity," Adv. Theor. Math. Phys. 2 (1998) 231 [Int. J. Theor. Phys. 38 (1999) 1113] [arXiv:hep-th/9711200].

[21] O. Aharony, S. S. Gubser, J. M. Maldacena, H. Ooguri and Y. Oz, "Large N field theories, string theory and gravity," Phys. Rept. 323 (2000) 183 arXiv:hep-th/9905111.

[22] A. Strominger and C. Vafa, "Microscopic Origin of the Bekenstein-Hawking Entropy," Phys. Lett. B 379 (1996) 99 [arXiv:hep-th/9601029].

[23] J. R. David, G. Mandal and S. R. Wadia, "Microscopic formulation of black holes in string theory," Phys. Rept. 369 (2002) 549; arXiv:hep-th/0203048.

[24] T. Banks and L. J. Dixon, "Constraints On String Vacua With Space-Time Supersymmetry," Nucl. Phys. B 307 (1988) 93.

[25] I. Antoniadis, C. Bachas, D. C. Lewellen and T. N. Tomaras, "On Supersymmetry Breaking In Superstrings," Phys. Lett. B 207 (1988) 441.

[26] I. Antoniadis, "A Possible New Dimension At A Few Tev," Phys. Lett. B 246 (1990) 377.

[27] E. Kiritsis, C. Kounnas, M. Petropoulos and J. Rizos "Solving the compactification problem in String Theory" [arXiv:hep-th/9606087]

[28] E. Witten, "Strong Coupling Expansion Of Calabi-Yau Compactification," Nucl. Phys. B 471 (1996) 135 arXiv:hep-th/9602070.

[29] J. D. Lykken, "Weak Scale Superstrings," Phys. Rev. D 54 (1996) 3693; arXiv:hep-th/9603133.

[30] N. Arkani-Hamed, S. Dimopoulos and G. R. Dvali, "The hierarchy problem and new dimensions at a millimeter," Phys. Lett. B 429 (1998) 263 [arXiv:hep-ph/9803315.

[31] I. Antoniadis, N. Arkani-Hamed, S. Dimopoulos and G. R. Dvali, "New dimensions at a millimeter to a Fermi and superstrings at a TeV," Phys. Lett. B 436 (1998) 257 arXiv:hep-ph/9804398. 
[32] C. P. Bachas, "Unification with low string scale," JHEP 9811 (1998) 023; [arXiv:hep-ph/9807415].

[33] T. Regge and C. Teitelboim, "General Relativity A La String: A Progress Report. (Talk)," SPIRES entry

[34] V. Rubakov and M. Shaposhnikov, "Do We Live Inside A Domain Wall?," Phys. Lett. B125 (1983) 136.

[35] K. Akama, "An Early Proposal Of 'Brane World'," Lect. Notes Phys. 176 (1982) 267 [arXiv:hep-th/0001113.

[36] L. Randall and R. Sundrum, "A large mass hierarchy from a small extra dimension," Phys. Rev. Lett. 83 (1999) 3370 arXiv:hep-ph/9905221.

[37] L. Randall and R. Sundrum, "An alternative to compactification," Phys. Rev. Lett. 83 (1999) 4690 arXiv:hep-th/9906064.

[38] P. Binetruy, C. Deffayet and D. Langlois, "Non-conventional cosmology from a braneuniverse," Nucl. Phys. B565 (2000) 269; arXiv:hep-th/9905012.

[39] J. M. Cline, C. Grojean and G. Servant, "Cosmological expansion in the presence of extra dimensions," Phys. Rev. Lett. 83 (1999) 4245; arXiv:hep-ph/9906523.

[40] P. Binetruy, C. Deffayet, U. Ellwanger and D. Langlois, "Brane cosmological evolution in a bulk with cosmological constant," Phys. Lett. B 477 (2000) 285;

[arXiv:hep-th/9910219].

[41] A. Kehagias and E. Kiritsis, "Mirage cosmology," JHEP 9911 (1999) 022; [arXiv:hep-th/9910174.

[42] S. Alexander, R. H. Brandenberger and D. Easson, "Brane gases in the early universe," Phys. Rev. D 62 (2000) 103509 [arXiv:hep-th/0005212].

[43] L. Cornalba, M. S. Costa and C. Kounnas, "A resolution of the cosmological singularity with orientifolds," Nucl. Phys. B 637 (2002) 378 [arXiv:hep-th/0204261].

[44] S. Kachru, X. Liu, M. B. Schulz and S. P. Trivedi, "Supersymmetry changing bubbles in string theory," arXiv:hep-th/0205108].

[45] C. P. Burgess, F. Quevedo, S. J. Rey, G. Tasinato and I. Zavala, "Cosmological spacetimes from negative tension brane backgrounds," JHEP 0210 (2002) 028; arXiv:hep-th/0207104].

[46] C. P. Burgess, M. Majumdar, D. Nolte, F. Quevedo, G. Rajesh and R. J. Zhang, "The inflationary brane-antibrane universe," JHEP 0107 (2001) 047;

arXiv:hep-th/0105204. 
[47] C. P. Burgess, P. Martineau, F. Quevedo, G. Rajesh and R. J. Zhang, "Brane antibrane inflation in orbifold and orientifold models," JHEP 0203 (2002) 052; [arXiv:hep-th/0111025].

[48] S. Watson and R. H. Brandenberger, "Isotropization in brane gas cosmology," Phys. Rev. D 67 (2003) 043510 [arXiv:hep-th/0207168.

[49] J. Khoury, B. A. Ovrut, N. Seiberg, P. J. Steinhardt and N. Turok, "From big crunch to big bang," Phys. Rev. D 65 (2002) 086007 [arXiv:hep-th/0108187].

[50] G. R. Dvali, Q. Shafi and S. Solganik, "D-brane inflation," [arXiv:hep-th/0105203.

[51] G. R. Dvali, G. Gabadadze and M. Porrati, "Metastable gravitons and infinite volume extra dimensions," Phys. Lett. B 484 (2000) 112; arXiv:hep-th/0002190].

[52] G. Dvali, G. Gabadadze and M. Porrati, "4D gravity on a brane in 5D Minkowski space," Phys. Lett. B485 (2000) 208:[arXiv:hep-th/0005016].

[53] G. Dvali and G. Gabadadze, "Gravity on a brane in infinite-volume extra space," Phys. Rev. D63 (2001) 065007; arXiv:hep-th/0008054.

[54] M.Carena, A. Delgado, J. Lykken, S. Pokorski, M. Quiros and C. Wagner, "Brane effects on extra dimensional scenarios: A tale of two gravitons," Nucl. Phys. B609 (2001) 499 [arXiv:hep-ph/0102172].

[55] C. Deffayet, G. Dvali, G. Gabadadze and A. Vainshtein, "Nonperturbative continuity in graviton mass versus perturbative discontinuity," [arXiv:hep-th/0106001].

[56] E. Kiritsis, G. Kofinas, N. Tetradis, T. N. Tomaras and V. Zarikas, "Cosmological evolution with brane-bulk energy exchange," JHEP 0302 (2003) 035;

[arXiv:hep-th/0207060].

[57] N. Tetradis, "Cosmological acceleration from energy influx," Phys. Lett. B 569 (2003) $1 ;$ arXiv:hep-th/0211200].

[58] P. S. Apostolopoulos and N. Tetradis, "Brane Cosmology with Matter in the Bulk," [arXiv:hep-th/0404105].

[59] T. N. Tomaras, "Brane-world evolution with brane-bulk energy exchange," arXiv:hep-th/0404142.

[60] G. W. Gibbons and C. A. Herdeiro, "Born-Infeld theory and stringy causality," Phys. Rev. D 63 (2001) 064006 arXiv:hep-th/0008052].

[61] G. Gibbons, K. Hashimoto and P. Yi, "Tachyon condensates, Carrollian contraction of Lorentz group, and fundamental strings," JHEP 0209 (2002) 061 [arXiv:hep-th/0209034.

[62] E. Verlinde, "On the holographic principle in a radiation dominated universe," arXiv:hep-th/0008140. 
[63] S. H. Alexander, "Inflation from D - anti-D brane annihilation," Phys. Rev. D 65 (2002) 023507 arXiv:hep-th/0105032.

[64] P. Horava and E. Witten, "Heterotic and type I string dynamics from eleven dimensions," Nucl. Phys. B 460 (1996) 506 arXiv:hep-th/9510209.

[65] A. Sen, "An introduction to non-perturbative string theory," arXiv:hep-th/9802051.

[66] E. Kiritsis, "Duality and instantons in string theory," arXiv:hep-th/9906018.

[67] C. Bachas, C. Fabre, E. Kiritsis, N. A. Obers and P. Vanhove, "Heterotic/type-I duality and D-brane instantons," Nucl. Phys. B 509 (1998) 33 arXiv:hep-th/9707126].

[68] E. Kiritsis and N. A. Obers, "Heterotic/type-I duality in $D<10$ dimensions, threshold corrections and D-instantons," JHEP 9710 (1997) 004 arXiv:hep-th/9709058.

[69] E. Gava, A. Hammou, J. F. Morales and K. S. Narain, "On the perturbative corrections around D-string instantons," JHEP 9903 (1999) 023 arXiv:hep-th/9902202.

[70] A. B. Hammou and J. F. Morales, "Fivebrane instantons and higher derivative couplings in type I theory," Nucl. Phys. B 573 (2000) 335 [arXiv:hep-th/9910144].

[71] I. Antoniadis and B. Pioline, "Low-scale closed strings and their duals," Nucl. Phys. B 550 (1999) 41 arXiv:hep-th/9902055.

[72] I. Antoniadis, K. Benakli and A. Laugier, "D-brane models with non-linear supersymmetry," Nucl. Phys. B 631 (2002) 3 arXiv:hep-th/0111209/.

[73] I. Antoniadis, K. Benakli, A. Laugier and T. Maillard, "Brane to bulk supersymmetry breaking and radion force at micron distances," Nucl. Phys. B 662 (2003) 40 [arXiv:hep-ph/0211409].

[74] E. Kiritsis, N. A. Obers and B. Pioline, "Heterotic/type II triality and instantons on K3," JHEP 0001 (2000) 029 [arXiv:hep-th/0001083].

[75] S. Kachru and C. Vafa, "Exact results for N=2 compactifications of heterotic strings," Nucl. Phys. B 450 (1995) 69 [arXiv:hep-th/9505105].

[76] I. Antoniadis, S. Dimopoulos and A. Giveon, "Little string theory at a TeV," JHEP 0105 (2001) 055 arXiv:hep-th/0103033.

[77] D. Kutasov, "Introduction to little string theory," PDF file SPIRES entry Prepared for ICTP Spring School on Superstrings and Related Matters, Trieste, Italy, 2-10 Apr 2001

[78] C. Angelantonj and A. Sagnotti, "Open strings," Phys. Rept. 371 (2002) 1; [arXiv:hep-th/0204089].

[79] A. Dabholkar, "Lectures on orientifolds and duality," arXiv:hep-th/9804208. 
[80] C. Bachas, "A Way to break supersymmetry," arXiv:hep-th/9503030.

[81] M. Berkooz, M. R. Douglas and R. G. Leigh, "Branes intersecting at angles," Nucl. Phys. B 480 (1996) 265 [arXiv:hep-th/9606139].

[82] R. Blumenhagen, L. Goerlich, B. Kors and D. Lüst, "Noncommutative compactifications of type I strings on tori with magnetic background flux," JHEP 0010 (2000) 006 [arXiv:hep-th/0007024.

[83] R. Blumenhagen, B. Kors, D. Lüst and T. Ott, "The standard model from stable intersecting brane world orbifolds," Nucl. Phys. B 616 (2001) 3 arXiv:hep-th/0107138.

[84] R. Blumenhagen, B. Kors and D. Lüst, "Type I strings with F- and B-flux," JHEP 0102 (2001) 030 arXiv:hep-th/0012156/.

[85] C. Angelantonj, I. Antoniadis, E. Dudas and A. Sagnotti, "Type-I strings on magnetised orbifolds and brane transmutation," Phys. Lett. B 489 (2000) 223; arXiv:hep-th/0007090].

[86] M. R. Douglas and G. W. Moore, "D-branes, Quivers, and ALE Instantons," arXiv:hep-th/9603167.

[87] G. Aldazabal, L. E. Ibãnez, F. Quevedo and A. M. Uranga, "D-branes at singularities: A bottom-up approach to the string embedding of the standard model," JHEP 0008 (2000) 002 [arXiv:hep-th/0005067.

[88] G. Aldazabal, S. Franco, L. E. Ibãnez, R. Rabadan and A. M. Uranga, "Intersecting brane worlds," JHEP 0102 (2001) 047; [arXiv:hep-ph/0011132].

[89] G. Aldazabal, S. Franco, L. E. Ibãnez, R. Rabadan and A. M. Uranga, "D = 4 chiral string compactifications from intersecting branes," J. Math. Phys. 42 (2001) 3103; [arXiv:hep-th/0011073].

[90] L. E. Ibãnez, F. Marchesano and R. Rabadan, "Getting just the standard model at intersecting branes," JHEP 0111 (2001) 002; [arXiv:hep-th/0105155].

[91] D. Cremades, L. E. Ibãnez and F. Marchesano, "Standard model at intersecting D5branes: Lowering the string scale," Nucl. Phys. B 643 (2002) 93; [arXiv:hep-th/0205074].

[92] M. Porrati, "Off-Shell Ward Identities And Gauge Symmetries In String Theory," Phys. Lett. B 231 (1989) 403.

[93] J. D. Blum and K. R. Dienes, "Duality without supersymmetry: The case of the SO(16) x SO(16) string," Phys. Lett. B 414 (1997) 260; arXiv:hep-th/9707148. "Strong/weak coupling duality relations for non-supersymmetric string theories," Nucl. Phys. B 516 (1998) 83 [arXiv:hep-th/9707160].

[94] Z. Kakushadze and S. H. H. Tye, "Brane world," Nucl. Phys. B 548 (1999) 180 arXiv:hep-th/9809147]. 
[95] I. Antoniadis, G. D'Appollonio, E. Dudas and A. Sagnotti, "Partial breaking of supersymmetry, open strings and M-theory," Nucl. Phys. B 553 (1999) 133; arXiv:hep-th/9812118;

[96] I. Antoniadis, G. D'Appollonio, E. Dudas and A. Sagnotti, "Open descendants of $Z_{2} \times Z_{2}$ freely-acting orbifolds," Nucl. Phys. B 565, 123 (2000) [arXiv:hep-th/9907184.

[97] I. Antoniadis, E. Dudas and A. Sagnotti, "Brane supersymmetry breaking," Phys. Lett. B 464 (1999) 38; arXiv:hep-th/9908023];

[98] G. Aldazabal and A. M. Uranga, "Tachyon-free non-supersymmetric type IIB orientifolds via brane-antibrane systems," JHEP 9910, 024 (1999) arXiv:hep-th/9908072.

[99] C. Angelantonj, I. Antoniadis, G. D'Appollonio, E. Dudas and A. Sagnotti, "Type I vacua with brane supersymmetry breaking," Nucl. Phys. B 572 (2000) 36; [arXiv:hep-th/9911081.

[100] A. L. Cotrone, "A $Z_{2} \times Z_{2}$ orientifold with spontaneously broken supersymmetry," Mod. Phys. Lett. A 14 (1999) 2487 [arXiv:hep-th/9909116].

[101] C. A. Scrucca, M. Serone and M. Trapletti, "Open string models with Scherk-Schwarz SUSY breaking and localized anomalies," Nucl. Phys. B 635, 33 (2002) [arXiv:hep-th/0203190].

[102] P. Anastasopoulos, A. B. Hammou and N. Irges, "A class of non-supersymmetric open string vacua," Phys. Lett. B 581 (2004) 248 [arXiv:hep-th/0310277].

[103] C. A. Scrucca and M. Serone, "Anomalies in field theories with extra dimensions," arXiv:hep-th/0403163.

[104] D. Cremades, L. E. Ibãnez and F. Marchesano, "SUSY quivers, intersecting branes and the modest hierarchy problem," JHEP 0207 (2002) 009;

arXiv:hep-th/0201205].

[105] T. Kugo and S. Uehara, "Conformal And Poincare Tensor Calculi In N=1 Supergravity," Nucl. Phys. B 226 (1983) 49; "On The 'New Nonminimal Versions' Of N=1 Poincare Supergravity," Nucl. Phys. B 226 (1983) 93.

[106] J. Polchinski and Y. Cai, "Consistency Of Open Superstring Theories," Nucl. Phys. B 296 (1988) 91.

[107] D. Cremades, L. E. Ibãnez and F. Marchesano, "Intersecting brane models of particle physics and the Higgs mechanism," JHEP 0207 (2002) 022 [arXiv:hep-th/0203160].

[108] I. Antoniadis, E. Kiritsis and T. N. Tomaras, "A D-brane alternative to unification," Phys. Lett. B 486 (2000) 186 [arXiv:hep-ph/0004214];

"D-brane Standard Model," Fortsch. Phys. 49 (2001) 573 [arXiv:hep-th/0111269]. 
[109] I. Antoniadis, E. Kiritsis, J. Rizos and T. N. Tomaras, "D-branes and the standard model," Nucl. Phys. B 660 (2003) 81; arXiv:hep-th/0210263].

[110] R. Blumenhagen, D. Lüst and S. Stieberger, "Gauge unification in supersymmetric intersecting brane worlds," JHEP 0307 (2003) 036; [arXiv:hep-th/0305146].

[111] H. Arason, D. J. Castano, B. Keszthelyi, S. Mikaelian, E. J. Piard, P. Ramond and B. D. Wright, "Renormalization group study of the standard model and its extensions. 1. The Standard model," Phys. Rev. D 46 (1992) 3945.

[112] M. K. Parida and A. Usmani, "Quark-lepton Yukawa-unification at lower mass scales," Phys. Rev. D 54 (1996) 3663.

[113] D. M. Ghilencea, L. E. Ibãnez, N. Irges and F. Quevedo, "TeV-scale Z' bosons from D-branes," JHEP 0208 (2002) 016 [arXiv:hep-ph/0205083].

[114] E. Kiritsis and P. Anastasopoulos, "The anomalous magnetic moment of the muon in the D-brane realization of the standard model," JHEP 0205 (2002) 054 arXiv:hep-ph/0201295/.

[115] A. Sagnotti, "A Note on the Green-Schwarz mechanism in open string theories," Phys. Lett. B 294 (1992) 196 [arXiv:hep-th/9210127].

[116] L. E. Ibãnez, R. Rabadan and A. M. Uranga, "Anomalous U(1)'s in type I and type $\mathrm{IIB} \mathrm{D}=4, \mathrm{~N}=1$ string vacua," Nucl. Phys. B 542 (1999) 112 [arXiv:hep-th/9808139.

[117] I. Antoniadis, C. Bachas and E. Dudas, "Gauge couplings in four-dimensional type I string orbifolds," Nucl. Phys. B 560 (1999) 93;

[arXiv:hep-th/9906039.

[118] E. Poppitz, "On the one loop Fayet-Iliopoulos term in chiral four dimensional type I orbifolds," Nucl. Phys. B 542 (1999) 31 [arXiv:hep-th/9810010].

[119] C. P. Burgess, L. E. Ibãnez and F. Quevedo, "Strings at the intermediate scale or is the Fermi scale dual to the Planck scale?," Phys. Lett. B 447 (1999) 257 [arXiv:hep-ph/9810535.

[120] L. E. Ibãnez and F. Quevedo, "Anomalous U(1)'s and proton stability in brane models," JHEP 9910 (1999) 001 [arXiv:hep-ph/9908305].

[121] G. 't Hooft, "How Instantons Solve The U(1) Problem," Phys. Rept. 142 (1986) 357.

[122] C. A. Scrucca, M. Serone and M. Trapletti, "Open string models with Scherk-Schwarz SUSY breaking and localized anomalies," Nucl. Phys. B 635 (2002) 33; [arXiv:hep-th/0203190].

[123] I. Antoniadis, E. Kiritsis and J. Rizos, "Anomalous U(1)s in type I superstring vacua," Nucl. Phys. B 637 (2002) 92; arXiv:hep-th/0204153. 
[124] P. Anastasopoulos, "Anomalous U(1)s masses in non-supersymmetric open string vacua," Phys. Lett. B 588 (2004) 119 [arXiv:hep-th/0402105].

[125] M. Klein, "Anomaly cancellation in $\mathrm{D}=4, \mathrm{~N}=1$ orientifolds and linear/chiral multiplet duality," Nucl. Phys. B 569 (2000) 362 [arXiv:hrep-th/9910143.

[126] I. Antoniadis, C. Bachas and E. Dudas, "Gauge couplings in four-dimensional type I string orbifolds," Nucl. Phys. B 560 (1999) 93; arXiv:hep-th/9906039].

[127] G. Aldazabal, A. Font, L. E. Ibãnez and G. Violero, "D = 4, N = 1, type IIB orientifolds," Nucl. Phys. B 536 (1998) 29 [arXiv:hep-th/9804026].

[128] P. Anastasopoulos, "4D anomalous U(1)'s, their masses and their relation to 6D anomalies," JHEP 0308 (2003) 005 [arXiv:hep-th/0306042].

[129] I. Antoniadis and C. Bachas, "Branes and the gauge hierarchy," Phys. Lett. B 450 (1999) 83 [arXiv:hep-th/9812093.

[130] K. R. Dienes, E. Dudas and T. Gherghetta, "Light neutrinos without heavy mass scales: A higher-dimensional seesaw mechanism," Nucl. Phys. B 557 (1999) 25 [arXiv:hep-ph/9811428.

[131] N. Arkani-Hamed, S. Dimopoulos, G. R. Dvali and J. March-Russell, "Neutrino masses from large extra dimensions," Phys. Rev. D 65 (2002) 024032 [arXiv:hep-ph/9811448.

[132] A. E. Faraggi and M. Pospelov, "Phenomenological issues in TeV scale gravity with light neutrino masses," Phys. Lett. B 458 (1999) 237 [arXiv:hep-ph/9901299.

[133] G. R. Dvali and A. Y. Smirnov, "Probing large extra dimensions with neutrinos," Nucl. Phys. B 563 (1999) 63 [arXiv:hep-ph/9904211].

[134] R. N. Mohapatra, S. Nandi and A. Perez-Lorenzana, "Neutrino masses and oscillations in models with large extra dimensions," Phys. Lett. B466 (1999) 115, [arXiv:hep-ph/9907520].

[135] R. N. Mohapatra and A. Perez-Lorenzana, "Sterile neutrino as a bulk neutrino," Nucl. Phys. B 576 (2000) 466 [arXiv:hep-ph/9910474].

[136] R. Barbieri, P. Creminelli and A. Strumia, "Neutrino oscillations from large extra dimensions," Nucl. Phys. B 585 (2000) 28 arXiv:hep-ph/0002199].

[137] G. Aldazabal, L. E. Ibãnez and F. Quevedo, "Standard-like models with broken supersymmetry from type I string vacua," JHEP 0001 (2000) 031 arXiv:hep-th/9909172.

[138] G. Aldazabal, L. E. Ibãnez and F. Quevedo, "A D-brane alternative to the MSSM," JHEP 0002 (2000) 015 arXiv:hep-ph/0001083.

[139] R. Blumenhagen, B. Kors and D. Lüst, "Moduli stabilization for intersecting brane worlds in type 0' string theory," Phys. Lett. B 532 (2002) 141 arXiv:hep-th/0202024. 
[140] J. R. Ellis, P. Kanti and D. V. Nanopoulos, "Intersecting branes flip SU(5)," Nucl. Phys. B 647, 235 (2002) [arXiv:hep-th/0206087].

[141] C. Kokorelis, "GUT model hierarchies from intersecting branes," JHEP 0208 (2002) 018 [arXiv:hep-th/0203187]; "New standard model vacua from intersecting branes," JHEP 0209 (2002) 029 [arXiv:hep-th/0205147]; "Exact standard model compactifications from intersecting branes," JHEP 0208 (2002) 036 [arXiv:hep-th/0206108]; "Exact standard model structures from intersecting D5-branes," Nucl. Phys. B 677 (2004) 115 [arXiv:hep-th/0207234]; "Deformed intersecting D6-brane GUTs. I," JHEP 0211 (2002) 027 arXiv:hep-th/0209202.

[142] A. M. Uranga, "Local models for intersecting brane worlds," JHEP 0212 (2002) 058 [arXiv:hep-th/0208014].

[143] D. Bailin, G. V. Kraniotis and A. Love, "New standard-like models from intersecting D4-branes," Phys. Lett. B 547 (2002) 43 [arXiv:hep-th/0208103]. "Standard-like models from intersecting D5-branes," Phys. Lett. B 553 (2003) 79 arXiv:hep-th/0210219; "Intersecting D5-brane models with massive vector-like leptons," JHEP 0302 (2003) 052 [arXiv:hep-th/0212112].

[144] M. Axenides, E. Floratos and C. Kokorelis, "SU(5) unified theories from intersecting branes," JHEP 0310 (2003) 006 [arXiv:hep-th/0307255].

[145] R. Blumenhagen, V. Braun, B. Kors and D. Lüst, "Orientifolds of K3 and Calabi-Yau manifolds with intersecting D-branes," JHEP 0207 (2002) 026 [arXiv:hep-th/0206038.

[146] J. F. G. Cascales and A. M. Uranga, "Chiral 4d N = 1 string vacua with D-branes and NSNS and RR fluxes," JHEP 0305 (2003) 011 [arXiv:hep-th/0303024].

[147] G. Honecker, "Chiral supersymmetric models on an orientifold of $Z_{4} \times Z_{2}$ with intersecting D6-branes," Nucl. Phys. B 666 (2003) 175 [arXiv:hep-th/0303015.

[148] R. Blumenhagen, D. Lüst and T. R. Taylor, "Moduli stabilization in chiral type IIB orientifold models with fluxes," arXiv:hep-th/0303016.

[149] J. F. G. Cascales, M. P. Garcia del Moral, F. Quevedo and A. M. Uranga, "Realistic D-brane models on warped throats: Fluxes, hierarchies and moduli stabilization," JHEP 0402 (2004) 031 arXiv:hep-th/0312051.

[150] T. P. T. Dijkstra, L. R. Huiszoon and A. N. Schellekens, "Chiral supersymmetric standard model spectra from orientifolds of Gepner models," arXiv:hep-th/0403196.

[151] G. Honecker and T. Ott, "Getting just the supersymmetric standard model at intersecting branes on the $Z_{6}$-orientifold," arXiv:hep-th/0404055.

[152] M. Cvetic, G. Shiu and A. M. Uranga, "Three-family supersymmetric standard like models from intersecting brane worlds," Phys. Rev. Lett. 87 (2001) 201801 
arXiv:hep-th/0107143]; "Chiral four-dimensional N = 1 supersymmetric type IIA orientifolds from intersecting D6-branes," Nucl. Phys. B 615 (2001) 3

[arXiv:hep-th/0107166].

[153] R. Blumenhagen, L. Gorlich and T. Ott, "Supersymmetric intersecting branes on the type IIA $T^{6} / Z_{4}$ orientifold," JHEP 0301 (2003) 021 [arXiv:hep-th/0211059].

[154] M. Cvetic, I. Papadimitriou and G. Shiu, "Supersymmetric three family SU(5) grand unified models from type IIA orientifolds with intersecting D6-branes," [arXiv:hep-th/0212177].

[155] M. Cvetic and I. Papadimitriou, "More supersymmetric standard-like models from intersecting D6-branes on type IIA orientifolds," [arXiv:hep-th/0303197].

[156] M. Cvetic, P. Langacker and G. Shiu, "Phenomenology of a three-family standardlike string model," Phys. Rev. D 66 (2002) 066004 arXiv:hep-ph/0205252]; "A threefamily standard-like orientifold model: Yukawa couplings and hierarchy," Nucl. Phys. B 642 (2002) 139 arXiv:hep-th/0206115].

[157] M. Cvetic and I. Papadimitriou, "More supersymmetric standard-like models from intersecting D6-branes on type IIA orientifolds," Phys. Rev. D 67 (2003) 126006 arXiv:hep-th/0303197].

[158] M. Cvetic, P. Langacker and J. Wang, "Dynamical supersymmetry breaking in standard-like models with intersecting D6-branes," Phys. Rev. D 68 (2003) 046002 arXiv:hep-th/0303208.

[159] D. Lüst and S. Stieberger, "Gauge threshold corrections in intersecting brane world models," arXiv:hep-th/0302221].

[160] D. Cremades, L. E. Ibãnez and F. Marchesano, "Yukawa couplings in intersecting D-brane models," JHEP 0307 (2003) 038 arXiv:hep-th/0302105].

[161] S. A. Abel and A. W. Owen, "Interactions in intersecting brane models," Nucl. Phys. B 663 (2003) 197 arXiv:hep-th/0303124].

[162] B. Kors and P. Nath, "Effective action and soft supersymmetry breaking for intersecting D-brane models," Nucl. Phys. B 681 (2004) 77 arXiv:hep-th/0309167.

[163] S. A. Abel, O. Lebedev and J. Santiago, "Flavour in intersecting brane models and bounds on the string scale," arXiv:hep-ph/0312157].

[164] D. Lüst, P. Mayr, R. Richter and S. Stieberger, "Scattering of gauge, matter, and moduli fields from intersecting branes," arXiv:hep-th/0404134].

[165] D. Cremades, L. E. Ibãnez and F. Marchesano, "Computing Yukawa couplings from magnetized extra dimensions," arXiv:hep-th/0404229.

[166] R. Maartens, "Brane-world gravity," arXiv:gr-qc/0312059. 
[167] C. Csaki, "TASI lectures on extra dimensions and branes," arXiv:hep-ph/0404096.

[168] P. Brax, C. van de Bruck and A. C. Davis, "Brane world cosmology," arXiv:hepth/0404011].

[169] J. Garriga and T. Tanaka, "Gravity in the brane-world," Phys. Rev. Lett. 84 (2000) 2778 arXiv:hep-th/9911055].

[170] S. B. Giddings, E. Katz and L. Randall, "Linearized gravity in brane backgrounds," JHEP 0003 (2000) 023 [arXiv:hep-th/0002091.

[171] N. Deruelle and T. Dolezel, "On linearized gravity in the Randall-Sundrum scenario," Phys. Rev. D 64 (2001) 103506 [arXiv:gr-qc/0105118].

[172] E. Kiritsis, N. Tetradis and T. N. Tomaras, "Induced gravity on RS branes," JHEP 0203 (2002) 019 arXiv:hep-th/0202037.

[173] Z. Kakushadze, "Gravity in Randall-Sundrum brane world revisited," Phys. Lett. B 497 (2001) 125 arXiv:hep-th/0008128.

[174] M. Visser, "Sakharov's induced gravity: A modern perspective," Mod. Phys. Lett. A 17 (2002) 977 [arXiv:gr-qc/0204062].

[175] S. Corley, D. A. Lowe and S. Ramgoolam, "Einstein-Hilbert action on the brane for the bulk graviton," JHEP 0107 (2001) 030 [arXiv:hep-th/0106067.

[176] I. Antoniadis, E. Gava and K. S. Narain, "Moduli corrections to gravitational couplings from string loops," Phys. Lett. B 283 (1992) 209 arXiv:hep-th/9203071.

[177] E. Kiritsis and C. Kounnas, "Infrared regularization of superstring theory and the one loop calculation of coupling constants," Nucl. Phys. B 442 (1995) 472;

[arXiv:hep-th/9501020]; "Curved four-dimensional space-times as infrared regulator in superstring theories," Nucl. Phys. Proc. Suppl. 41 (1995) 331 arXiv:hep-th/9410212].

[178] E. Kiritsis, "Introduction to superstring theory,", Leuven University Press (1998), [arXiv:hep-th/9709062].

[179] I. Antoniadis, S. Ferrara, R. Minasian and K. S. Narain, " $R^{4}$ couplings in M- and type II theories on Calabi-Yau spaces," Nucl. Phys. B 507 (1997) 571; arXiv:hep-th/9707013.

[180] E. Kohlprath, "Renormalization of the Planck mass for type II superstrings on symmetric orbifolds," JHEP 0210 (2002) 026; arXiv:hep-th/0207023].

[181] I. Antoniadis, R. Minasian and P. Vanhove, "Non-compact Calabi-Yau manifolds and localized gravity," Nucl. Phys. B 648 (2003) 69; [arXiv:hep-th/0209030].

[182] I. Antoniadis, C. Bachas, C. Fabre, H. Partouche and T. R. Taylor, "Aspects of type I - type II - heterotic triality in four dimensions," Nucl. Phys. B 489 (1997) 160 [arXiv:hep-th/9608012]. 
[183] E. Kiritsis, N. Tetradis and T. N. Tomaras, "Brane Induced Gravity: Realizations and Limitations" JHEP 0108 (2001) 012; [arXiv:hep-th/0106050].

[184] E. Kohlprath, "Induced gravity in Z(N) orientifold models," arXiv:hep-th/0311251].

[185] G. Dvali, G. Gabadadze, M. Kolanovic and F. Nitti, "Scales of gravity," Phys. Rev. D64 (2001) 084004 arXiv:hep-ph/0102216.

[186] S. L. Dubovsky and V. A. Rubakov, "On models of gauge field localization on a brane," Int. J. Mod. Phys. A 16 (2001) 4331 arXiv:hep-th/0105243.

[187] Put here the papers of vainshtein et al

[188] A. Gruzinov, "On the graviton mass," arXiv:astro-ph/0112246].

[189] A. Lue, "Cosmic strings in a brane world theory with metastable gravitons," Phys. Rev. D 66 (2002) 043509 arXiv:hep-th/0111168].

[190] M. Porrati, "Fully covariant van Dam-Veltman-Zakharov discontinuity, and absence thereof," Phys. Lett. B 534 (2002) 209 [arXiv:hep-th/0203014].

[191] M. A. Luty, M. Porrati and R. Rattazzi, "Strong interactions and stability in the DGP model," JHEP 0309 (2003) 029 [arXiv:hep-th/0303116].

[192] V. A. Rubakov, "Strong coupling in brane-induced gravity in five dimensions," arXiv:hep-th/0303125.

[193] C. Deffayet, G. R. Dvali, G. Gabadadze and A. I. Vainshtein, "Nonperturbative continuity in graviton mass versus perturbative discontinuity," Phys. Rev. D 65 (2002) 044026 [arXiv:hep-th/0106001.

[194] O. Corradini, A. Iglesias, Z. Kakushadze and P. Langfelder, "Gravity on a 3-brane in 6D bulk," Phys. Lett. B 521 (2001) 96 [arXiv:hep-th/0108055].

[195] I. Giannakis and H. c. Ren, "Linearized analysis of the Dvali-Gabadadze-Porrati brane model," Phys. Lett. B 528 (2002) 133 arXiv:hep-th/0111127.

[196] C. Deffayet, S. J. Landau, J. Raux, M. Zaldarriaga and P. Astier, "Supernovae, CMB, and gravitational leakage into extra dimensions," Phys. Rev. D 66 (2002) 024019 [arXiv:astro-ph/0201164].

[197] C. Deffayet, "On brane world cosmological perturbations," Phys. Rev. D 66 (2002) 103504 arXiv:hep-th/0205084.

[198] G. Kofinas, E. Papantonopoulos and V. Zamarias, "Black hole solutions in braneworlds with induced gravity," Phys. Rev. D 66 (2002) 104028 arXiv:hep-th/0208207].

[199] C. Middleton and G. Siopsis, "Fat branes in infinite volume extra space," arXiv:hep-th/0210033. 
[200] M. Kolanovic, "Gravity induced over a smooth soliton," Phys. Rev. D 67 (2003) 106002 arXiv:hep-th/0301116].

[201] K. i. Maeda, S. Mizuno and T. Torii, "Effective gravitational equations on brane world with induced gravity," Phys. Rev. D 68 (2003) 024033 [arXiv:gr-qc/0303039].

[202] U. Ellwanger, "Blown-up p-branes and the cosmological constant," JCAP 0311 (2003) 013 [arXiv:hep-th/0304057].

[203] M. Kolanovic, M. Porrati and J. W. Rombouts, "Regularization of brane induced gravity," Phys. Rev. D 68 (2003) 064018 [arXiv:hep-th/0304148.

[204] R. Gregory, "Inflating p-branes," JHEP 0306 (2003) 041 [arXiv:hep-th/0304262.

[205] C. Charmousis, S. C. Davis and J. F. Dufaux, "Scalar brane backgrounds in higher order curvature gravity," JHEP 0312 (2003) 029 [arXiv:hep-th/0309083].

[206] S. L. Dubovsky and M. V. Libanov, "On brane-induced gravity in warped backgrounds," JHEP 0311 (2003) 038 [arXiv:hep-th/0309131].

[207] C. Middleton and G. Siopsis, "The Schwarzschild solution in the DGP model," [arXiv:hep-th/0311070].

[208] A. Karch and L. Randall, "Locally localized gravity," JHEP 0105 (2001) 008 arXiv:hep-th/0011156].

[209] T. Shiromizu, K. Maeda and M. Sasaki, "The Einstein equations on the 3-brane world," Phys. Rev. D 62 (2000) 024012; [arXiv:gr-qc/9910076].

[210] P. Kraus, "Dynamics of anti-de Sitter domain walls," JHEP 9912 (1999) 011 [arXiv:hep-th/9910149].

[211] C. Deffayet, "Cosmology on a brane in Minkowski bulk," Phys. Lett. B502 (2001) 199 [arXiv:hep-th/0010186].

[212] G. Kofinas, "General brane cosmology with $R_{4}$ term in $\mathrm{AdS}_{5}$ or Minkowski bulk," JHEP 0108 (2001) 034 arXiv:hep-th/0108013.

[213] J. E. Kim, B. Kyae and H. M. Lee, "Various modified solutions of the RandallSundrum model with the Gauss-Bonnet interaction," Nucl. Phys. B 582 (2000) 296 [Erratum-ibid. B 591 (2000) 587] [arXiv:hep-th/0004005].

[214] I. Low and A. Zee, "Naked singularity and Gauss-Bonnet term in brane world scenarios," Nucl. Phys. B 585 (2000) 395 [arXiv:hep-th/0004124].

[215] B. Abdesselam and N. Mohammedi, "Brane world cosmology with Gauss-Bonnet interaction," Phys. Rev. D 65 (2002) 084018 [arXiv:hep-th/0110143].

[216] C. Charmousis and J. F. Dufaux, "General Gauss-Bonnet brane cosmology," Class. Quant. Grav. 19 (2002) 4671 arXiv:hep-th/0202107. 
[217] N. Deruelle and M. Sasaki, "Newton's law on an Einstein 'Gauss-Bonnet' brane," Prog. Theor. Phys. 110 (2003) 441 [arXiv:gr-qc/0306032].

[218] G. Kofinas, R. Maartens and E. Papantonopoulos, "Brane cosmology with curvature corrections," JHEP 0310 (2003) 066 [arXiv:hep-th/0307138].

[219] J. F. Dufaux, J. E. Lidsey, R. Maartens and M. Sami, "Cosmological perturbations from brane inflation with a Gauss-Bonnet term," arXiv:hep-th/0404161.

[220] O. Corradini, "4D gravity on a brane from bulk higher-curvature terms," arXiv:hep-th/0405038.

[221] C. van de Bruck, M. Dorca, C. J. Martins and M. Parry, "Cosmological consequences of the brane/bulk interaction," Phys. Lett. B 495 (2000) 183 arXiv:hep-th/0009056.

[222] U. Ellwanger, "Cosmological evolution in compactified Horava-Witten theory induced by matter on the branes," Eur. Phys. J. C 25, 157 (2002) [arXiv:hep-th/0001126].

[223] A. Hebecker and J. March-Russel, "Randall-Sundrum II cosmology, AdS/CFT, and the bulk black hole," Nucl. Phys. B608 (2001) 375, [arXiv:hep-ph/0103214].

[224] P. Brax, C. van de Bruck and A. C. Davis, "Brane-world cosmology, bulk scalars and perturbations," JHEP 0110 (2001) 026 [arXiv:hep-th/0108215];

[225] D. Langlois, L. Sorbo and M. Rodriguez-Martinez, "Cosmology of a brane radiating gravitons into the extra dimension," Phys. Rev. Lett. 89 (2002) 171301 [arXiv:hep-th/0206146].

[226] D. Langlois and L. Sorbo, "Bulk gravitons from a cosmological brane," Phys. Rev. D 68 (2003) 084006 [arXiv:hep-th/0306281].

[227] E. Leeper, R. Maartens and C. F. Sopuerta, "Dynamics of radiating braneworlds," Class. Quant. Grav. 21 (2004) 1125; [arXiv:gr-qc/0309080].

[228] F. Hoyle and J.V. Narlikar, Proc. Royal Soc. A277 (1964) 1; Ann. Phys. 54 (1969) 207.

[229] T. Damour, F. Piazza and G. Veneziano, "Violations of the equivalence principle in a dilaton-runaway scenario," Phys. Rev. D 66 (2002) 046007 arXiv:hep-th/0205111.

[230] C. Deffayet, G. Dvali, G. Gabadadze and A. Lue, "Braneworld flattening by a cosmological constant," Phys. Rev. D64 (2001) 104002; arXiv:hep-th/0104201.

[231] C. Deffayet, G. Dvali and G. Gabadadze, "Accelerated universe from gravity leaking to extra dimensions," Phys. Rev. D 65 (2002) 044023 [arXiv:astro-ph/0105068]; "Comments on "A Supernova Brane Scan"," [arXiv:astro-ph/0106449].

[232] H.A. Chamblin and H.S. Reall, "Dynamic dilatonic domain walls," Nucl. Phys. B 562 (1999) 133 arXiv:hep-th/9903225. 
[233] A. Chamblin, M.J. Perry and H.S. Reall, "Non-BPS D8-branes and dynamic domain walls in massive IIA supergravities," JHEP 09 (1999) 014 , arXiv:hep-th/9908047.

[234] E. Kiritsis, "Supergravity, D-brane probes and thermal super Yang-Mills: A comparison," JHEP 9910 (1999) 010 [arXiv:hep-th/9906206].

[235] F. Quevedo, "Lectures on string / brane cosmology," Class. Quant. Grav. 19 (2002) 5721 arXiv:hep-th/0210292].

[236] R. R. Caldwell, "A Phantom Menace?," Phys. Lett. B 545 (2002) 23 [arXiv:astro-ph/9908168].

[237] G. W. Gibbons, "Phantom matter and the cosmological constant," arXiv:hep-th/0302199.

[238] S. M. Carroll, M. Hoffman and M. Trodden, "Can the dark energy equation-of-state parameter w be less than -1?," Phys. Rev. D 68, 023509 (2003); arXiv:astro-ph/0301273.

[239] D.J. Chung and K. Freese, "Can geodesics in extra dimensions solve the cosmological horizon problem?," arXiv:hep-ph/991023 5.

[240] G. Kalbermann and H. Halevi, "Nearness through an extra dimension," [arXiv:gr-qc/9810083].

[241] G. Kalbermann, "Communication through an extra dimension," [arXiv:gr-qc/9910063].

[242] H. Ishihara, "Causality of the brane universe," Phys. Rev. Lett. 86 (2001) 381 [arXiv:gr-qc/0007070].

[243] R. R. Caldwell and D. Langlois, "Shortcuts in the fifth dimension," Phys. Lett. B 511 (2001) 129 [arXiv:gr-qc/0103070.

[244] E. Abdalla, B. Cuadros-Melgar, S. S. Feng and B. Wang, "The shortest cut in brane cosmology," Phys. Rev. D 65 (2002) 083512 arXiv:hep-th/0109024.

[245] J. Moffat, "Superluminary universe: A Possible solution to the initial value problem in cosmology," IJMP D2 (1993) 351, [arXiv:gr-qc/9211020].

[246] A. Albrecht and J. Magueijo, "A time varying speed of light as a solution to cosmological puzzles," Phys Rev. D59 (1999) 43516, arXiv:astro-ph/9811001.

[247] J. Barrow and J. Magueijo, "Solutions to the Quasi-flatness and Quasi-lambda Problems," Phys. Lett. B447 (1999) 246, arXiv:astro-ph/9811073]; "Varying- $\alpha$ Theories And Solutions To The Cosmological Problems," ibid. B443 (1998) 104;

arXiv:astro-ph/9811072. 
[248] D. Youm, "Brane world cosmologies with varying speed of light," Phys. Rev. D 63 (2001) 125011 arXiv:hep-th/0101228/.] "Variable-speed-of-light cosmology from brane world scenario," Phys. Rev. D 64 (2001) 085011 arXiv:hep-th/0102194];

[249] J. W. Moffat, "Acceleration of the universe, string theory and a varying speed of light," [arXiv:hep-th/0105017.

[250] E. Papantonopoulos and I. Pappa, "Type 0 brane inflation from mirage cosmology," Mod. Phys. Lett. A 15 (2000) 2145 arXiv:hep-th/0001183.

[251] C. Park and S. J. Sin, "Moving domain walls in AdS(5) and graceful exit from inflation," Phys. Lett. B 485 (2000) 239 [arXiv:hep-th/0005013].

[252] J. Y. Kim, "Brane inflation in tachyonic and non-tachyonic type 0B string theories," Phys. Rev. D 63 (2001) 045014 [arXiv:hep-th/0009175].

[253] E. Papantonopoulos and I. Pappa, "Cosmological evolution of a brane universe in a type 0 string background," Phys. Rev. D 63 (2001) 103506 [arXiv:hep-th/0010014.

[254] D. Youm, "Closed universe in mirage cosmology," Phys. Rev. D 63 (2001) 085010 [Erratum-ibid. D 63 (2001) 129902] [arXiv:hep-th/0011290].

[255] A. Kehagias and K. Tamvakis, "A note on brane cosmology," Phys. Lett. B 515 (2001) 155 [arXiv:hep-ph/0104195].

[256] M. F. Parry and D. A. Steer, "Brane gas inflation," JHEP 0202 (2002) 032 [arXiv:hep-ph/0109207].

[257] T. Boehm and D. A. Steer, "Perturbations on a moving D3-brane and mirage cosmology," Phys. Rev. D 66 (2002) 063510 [arXiv:hep-th/0206147].

[258] J. Y. Kim, "Mirage cosmology in M-theory," Phys. Lett. B 548 (2002) 1; [arXiv:hep-th/0203084].

[259] D. A. Steer and M. F. Parry, "Brane cosmology, varying speed of light and inflation in models with one or more extra dimensions," Int. J. Theor. Phys. 41 (2002) 2255 [arXiv:hep-th/0201121].

[260] E. Papantonopoulos and I. Pappa, "Inflation induced by vacuum energy and graceful exit from it," Mod. Phys. Lett. A 16 (2001) 2545 arXiv:gr-qc/0103101.

[261] G. Kofinas, "New perspectives on moving domain walls in (A)dS(5) space," Nucl. Phys. B 622 (2002) 347 [arXiv:hep-th/0103045].

[262] D. Youm, "Closed universe in mirage cosmology," Phys. Rev. D 63 (2001) 085010 [Erratum-ibid. D 63 (2001) 129902] [arXiv:hep-th/0011290].

[263] P. Brax and D. A. Steer, "Non-BPS brane cosmology," JHEP 0205 (2002) 016 arXiv:hep-th/0204120. 
[264] E. Kiritsis, unpublished, 1999.

[265] S. H. Alexander, "On the varying speed of light in a brane-induced FRW universe," JHEP 0011 (2000) 017 [arXiv:hep-th/9912037.

[266] P. Brax and D. A. Steer, "Remark on bouncing and cyclic branes in more than one extra dimension," Phys. Rev. D 66 (2002) 061501

[267] S. Kachru and L. McAllister, "Bouncing brane cosmologies from warped string compactifications," JHEP 0303 (2003) 018 [arXiv:hep-th/0205209].

[268] P. Kanti and K. Tamvakis, "Challenges and obstacles for a bouncing universe in brane models," Phys. Rev. D 68 (2003) 024014 [arXiv:hep-th/0303073].

[269] C. P. Burgess, P. Martineau, F. Quevedo and R. Rabadan, "Branonium," [arXiv:hep-th/0303170].

[270] E. Kiritsis and T. R. Taylor, "Thermodynamics of D-brane probes," arXiv:hep-th/9906048.

[271] N. Iizuka, D. Kabat, G. Lifschytz and D. A. Lowe, "Probing black holes in nonperturbative gauge theory," Phys. Rev. D 65 (2002) 024012 arXiv:hep-th/0108006.

[272] R. C. Myers, "Dielectric-branes," JHEP 9912 (1999) 022 [arXiv:hep-th/9910053.

[273] M. Gell-Mann and B. Zwiebach, "Curling Up Two Spatial Dimensions With SU $(1,1)$ / U(1)," Phys. Lett. B 147 (1984) 111; "Space-Time Compactification Due To Scalars," Phys. Lett. B 141 (1984) 333; "Dimensional Reduction Of Space-Time Induced By Nonlinear Scalar Dynamics And Noncompact Extra Dimensions,"

Nucl. Phys. B 260 (1985) 569.

[274] M. Visser, "An Exotic Class Of Kaluza-Klein Models," Phys. Lett. B 159 (1985) 22 arXiv:hep-th/9910093].

[275] G.W. Gibbons and D.L. Wiltshire, "Space-Time As A Membrane In Higher Dimensions," Nucl. Phys. B287 (1987) 717 arXiv:hep-th/0109093] and references therein.

[276] H. Verlinde, "Holography and compactification," Nucl. Phys. B 580 (2000) 264 arXiv:hep-th/9906182].

[277] Z. Kakushadze, "Orientiworld," JHEP 0110 (2001) 031 [arXiv:hep-th/0109054].

[278] O. Corradini, A. Iglesias, Z. Kakushadze and P. Langfelder, "Gravity on a 3-brane in 6D bulk," Phys. Lett. B 521 (2001) 96 [arXiv:hep-th/0108055.

[279] N. Arkani-Hamed, S. Dimopoulos and G. Dvali, "Phenomenology, Astrophysics and Cosmology of Theories with Sub-Millimeter Dimensions and TeV Scale Quantum Gravity", Phys. Rev. D59 (1999) 086004;arXiv:hep-ph/9807344]. 
[280] M. Trodden and S. M. Carroll, "TASI lectures: Introduction to cosmology," [arXiv:astro-ph/0401547].

[281] G. R. Dvali and S. H. H. Tye, "Brane inflation," Phys. Lett. B 450 (1999) 72 arXiv:hep-ph/9812483.

[282] G. W. Gibbons, "Cosmological evolution of the rolling tachyon," Phys. Lett. B 537 (2002) 1 arXiv:hep-th/0204008

[283] M. Fairbairn and M. H. G. Tytgat, "Inflation from a tachyon fluid?," Phys. Lett. B 546 (2002) 1 [arXiv:hep-th/0204070.

[284] S. Mukohyama, "Brane cosmology driven by the rolling tachyon," Phys. Rev. D 66 (2002) 024009 [arXiv:hep-th/0204084].

[285] A. Feinstein, "Power-law inflation from the rolling tachyon," Phys. Rev. D 66 (2002) 063511 arXiv:hep-th/0204140.

[286] A. V. Frolov, L. Kofman and A. A. Starobinsky, "Prospects and problems of tachyon matter cosmology," Phys. Lett. B 545 (2002) 8 [arXiv:hep-th/0204187].

[287] D. Choudhury, D. Ghoshal, D. P. Jatkar and S. Panda, "On the cosmological relevance of the tachyon," Phys. Lett. B 544 (2002) 231 [arXiv:hep-th/0204204].

[288] G. Shiu and I. Wasserman, "Cosmological constraints on tachyon matter," Phys. Lett. B 541 (2002) 6 arXiv:hep-th/0205003.

[289] L. Kofman and A. Linde, "Problems with tachyon inflation," JHEP 0207 (2002) 004 arXiv:hep-th/0205121.

[290] A. Sen, "Time evolution in open string theory," JHEP 0210 (2002) 003; [arXiv:hep-th/0207105.

[291] G. Shiu, S. H. H. Tye and I. Wasserman, "Rolling tachyon in brane world cosmology from superstring field theory," Phys. Rev. D 67 (2003) 083517 [arXiv:hep-th/0207119].

[292] J. M. Cline, H. Firouzjahi and P. Martineau, "Reheating from tachyon condensation," JHEP 0211 (2002) 041 arXiv:hep-th/0207156].

[293] M. C. Bento, O. Bertolami and A. A. Sen, "Tachyonic inflation in the braneworld scenario," Phys. Rev. D 67 (2003) 063511 [arXiv:hep-th/0208124].

[294] P. Mukhopadhyay and A. Sen, "Decay of unstable D-branes with electric field," JHEP 0211 (2002) 047 arXiv:hep-th/0208142.

[295] G. Shiu, "Tachyon dynamics and brane cosmology," [arXiv:hep-th/0210313.

[296] Z. K. Guo, Y. S. Piao, R. G. Cai and Y. Z. Zhang, "Inflationary attractor from tachyonic matter," Phys. Rev. D 68 (2003) 043508 arXiv:hep-ph/0304236. 
[297] S. Sarangi and S. H. H. Tye, "Inter-brane potential and the decay of a non-BPS-Dbrane to closed strings," Phys. Lett. B 573 (2003) 181 arXiv:hep-th/0307078.

[298] D. A. Steer and F. Vernizzi, "Tachyon inflation: Tests and comparison with single scalar field inflation," [arXiv:hep-th/0310139].

[299] F. Epple and D. Lüst, "Tachyon condensation for intersecting branes at small and large angles," Fortsch. Phys. 52 (2004) 367 [arXiv:hep-th/0311182].

[300] A. Sen, "Remarks on tachyon driven cosmology," [arXiv:hep-th/0312153].

[301] M. R. Garousi, M. Sami and S. Tsujikawa, "Inflation and dark energy arising from rolling massive scalar field on the D-brane," arXiv:hep-th/0402075].

[302] B. s. Kyae and Q. Shafi, "Branes and inflationary cosmology," Phys. Lett. B 526 (2002) 379 [arXiv:hep-ph/0111101].

[303] R. Blumenhagen, B. Kors, D. Lüst and T. Ott, "Hybrid inflation in intersecting brane worlds," Nucl. Phys. B 641 (2002) 235 [arXiv:hep-th/0202124].

[304] J. M. Cline and H. Firouzjahi, "Real-time D-brane condensation," Phys. Lett. B 564 (2003) 255 [arXiv:hep-th/0301101].

[305] S. Kachru, R. Kallosh, A. Linde and S. P. Trivedi, "De Sitter vacua in string theory," Phys. Rev. D 68 (2003) 046005 [arXiv:hep-th/0301240].

[306] M. Majumdar and A. C. Davis, "D-brane anti-brane annihilation in an expanding universe," JHEP 0312 (2003) 012 [arXiv:hep-th/0304153].

[307] R. Brandenberger, D. A. Easson and A. Mazumdar, "Inflation and brane gases," Phys. Rev. D 69 (2004) 083502 [arXiv:hep-th/0307043].

[308] S. Kachru, R. Kallosh, A. Linde, J. Maldacena, L. McAllister and S. P. Trivedi, "Towards inflation in string theory," JCAP 0310 (2003) 013 arXiv:hep-th/0308055.

[309] E. Dudas, J. Mourad and C. Timirgaziu, "On cosmologically induced hierarchies in string theory," JCAP 0403 (2004) 005 [arXiv:hep-th/0309057].

[310] G. Dvali and S. Kachru, "New old inflation," arXiv:hep-th/0309095].

[311] C. P. Burgess, N. E. Grandi, F. Quevedo and R. Rabadan, "D-brane chemistry," JHEP 0401 (2004) 067 arXiv:hep-th/0310010.

[312] E. Silverstein and D. Tong, "Scalar speed limits and cosmology: Acceleration from D-cceleration," arXiv:hep-th/0310221.

[313] J. P. Hsu, R. Kallosh and S. Prokushkin, "On brane inflation with volume stabilization," JCAP 0312 (2003) 009 arXiv:hep-th/0311077.

[314] A. Buchel and R. Roiban, "Inflation in warped geometries," arXiv:hep-th/0311154. 
[315] H. Firouzjahi and S. H. H. Tye, "Closer towards inflation in string theory," Phys. Lett. B 584 (2004) 147 arXiv:hep-th/0312020.

[316] L. Pilo, A. Riotto and A. Zaffaroni, "Old inflation in string theory," [arXiv:hep-th/0401004].

[317] P. Binetruy, G. Dvali, R. Kallosh and A. Van Proeyen, "Fayet-Iliopoulos terms in supergravity and cosmology," arXiv:hep-th/0402046].

[318] C. P. Burgess, J. M. Cline, H. Stoica and F. Quevedo, "Inflation in realistic D-brane models," [arXiv:hep-th/0403119.

[319] O. DeWolfe, S. Kachru and H. Verlinde, "The giant inflaton," JHEP 0405 (2004) 017 [arXiv:hep-th/0403123.

[320] N. Iizuka and S. P. Trivedi, "An inflationary model in string theory," arXiv:hep-th/0403203].

[321] N. Barnaby and J. M. Cline, "Creating the universe from brane-antibrane annihilation," arXiv:hep-th/0403223].

[322] M. Alishahiha, E. Silverstein and D. Tong, "DBI in the sky," arXiv:hep-th/0404084.

[323] M. Berg, M. Haack and B. Kors, "Loop corrections to volume moduli and inflation in string theory," arXiv:hep-th/0404087.

[324] A. Buchel and A. Ghodsi, "Braneworld inflation," [arXiv:hep-th/0404151].

[325] S. V. Babak and L. P. Grishchuk, "Finite-range gravity and its role in gravitational waves, black holes and cosmology," Int. J. Mod. Phys. D 12 (2003) 1905 [arXiv:gr-qc/0209006].

[326] T. Damour and I. I. Kogan, "Effective Lagrangians and universality classes of nonlinear bigravity," Phys. Rev. D 66 (2002) 104024 [arXiv:hep-th/0206042].

[327] T. Damour, I. I. Kogan and A. Papazoglou, "Non-linear bigravity and cosmic acceleration," Phys. Rev. D 66 (2002) 104025 [arXiv:hep-th/0206044].

[328] N. Arkani-Hamed, H. Georgi and M. D. Schwartz, "Effective field theory for massive gravitons and gravity in theory space," Annals Phys. 305 (2003) 96; [arXiv:hep-th/0210184].

[329] J. J. Atick and E. Witten, "The Hagedorn Transition And The Number Of Degrees Of Freedom Of String Theory," Nucl. Phys. B 310 (1988) 291.

[330] E. Witten, "Space-Time And Topological Orbifolds," Phys. Rev. Lett. 61 (1988) 670.

[331] D. Amati, M. Ciafaloni and G. Veneziano, "Superstring Collisions At Planckian Energies," Phys. Lett. B 197 (1987) 81; "Classical And Quantum Gravity Effects From Planckian Energy Superstring Collisions," Ænt. J. Mod. Phys. A 3 (1988) 1615. 
[332] D. J. Gross and P. F. Mende, "The High-Energy Behavior Of String Scattering Amplitudes," Phys. Lett. B 197 (1987) 129; "String Theory Beyond The Planck Scale," Nucl. Phys. B 303 (1988) 407;

[333] D. J. Gross, "High-Energy Symmetries Of String Theory," Phys. Rev. Lett. 60 (1988) 1229.

[334] P. F. Mende and H. Ooguri, "Borel Summation Of String Theory For Planck Scale Scattering," Nucl. Phys. B 339 (1990) 641.

[335] E. Witten, "Baryons In The 1/N Expansion," Nucl. Phys. B 160 (1979) 57.

[336] G. 't Hooft, "A Planar Diagram Theory For Strong Interactions," Nucl. Phys. B 72 (1974) 461.

[337] G. 't Hooft, "Dimensional Reduction In Quantum Gravity," [arXiv:gr-qc/9310026].

[338] L. Susskind, "The World as a hologram," J. Math. Phys. 36 (1995) 6377 arXiv:hep-th/9409089.

[339] R. L. Jaffe, K. Johnson and Z. Ryzak, "Qualitative Features Of The Glueball Spectrum," Annals Phys. 168 (1986) 344.

[340] R. Sundrum, "Towards an effective particle-string resolution of the cosmological constant problem," JHEP 9907 (1999) 001; [arXiv:hep-ph/9708329].

[341] "Fat gravitons, the cosmological constant and sub-millimeter tests,"Phys. Rev. D 69 (2004) 044014; arXiv:hep-th/0306106.

[342] A. Zee, "Dark energy and the nature of the graviton," arXiv:hep-th/0309032].

[343] N. Arkani-Hamed, A. G. Cohen and H. Georgi, "(De)constructing dimensions," Phys. Rev. Lett. 86 (2001) 4757 arXiv:hep-th/0104005].

[344] M. R. Douglas and S. H. Shenker, "Strings In Less Than One-Dimension," Nucl. Phys. B 335 (1990) 635.

[345] E. Brezin and V. A. Kazakov, "Exactly Solvable Field Theories Of Closed Strings," Phys. Lett. B 236 (1990) 144.

[346] D. J. Gross and A. A. Migdal, "Nonperturbative Two-Dimensional Quantum Gravity," Phys. Rev. Lett. 64 (1990) 127.

[347] T. Banks, W. Fischler, S. H. Shenker and L. Susskind, "M theory as a matrix model: A conjecture," Phys. Rev. D 55 (1997) 5112 arXiv:hep-th/9610043.

[348] N. Ishibashi, H. Kawai, Y. Kitazawa and A. Tsuchiya, "A large-N reduced model as superstring," Nucl. Phys. B 498 (1997) 467 [arXiv:hep-th/9612115].

[349] M. J. Teper, "Glueball masses and other physical properties of SU(N) gauge theories in $\mathrm{D}=3+1$ : A review of lattice results for theorists," [arXiv:hep-th/9812187]. 
[350] A. M. Polyakov, "The wall of the cave," Int. J. Mod. Phys. A 14 (1999) 645 [arXiv:hep-th/9809057].

[351] J. Polchinski and M. J. Strassler, "Hard scattering and gauge/string duality," Phys. Rev. Lett. 88 (2002) 031601 [arXiv:hep-th/0109174].

[352] T. Banks, M. R. Douglas, G. T. Horowitz and E. J. Martinec, "AdS dynamics from conformal field theory," arXiv:hep-th/9808016].

[353] V. Balasubramanian, P. Kraus, A. E. Lawrence and S. P. Trivedi, "Holographic probes of anti-de Sitter space-times," Phys. Rev. D 59 (1999) 104021 [arXiv:hep-th/9808017].

[354] P. Kraus, F. Larsen and S. P. Trivedi, "The Coulomb branch of gauge theory from rotating branes," JHEP 9903 (1999) 003 [arXiv:hep-th/9811120].

[355] S. R. Das, "Holograms of branes in the bulk and acceleration terms in SYM effective action," JHEP 9906 (1999) 029 arXiv:hep-th/9905037].

[356] I. R. Klebanov and M. J. Strassler, "Supergravity and a confining gauge theory: Duality cascades and chiSB-resolution of naked singularities," JHEP 0008 (2000) 052 [arXiv:hep-th/0007191].

[357] J. M. Maldacena and C. Nunez, "Supergravity description of field theories on curved manifolds and a no go theorem," Int. J. Mod. Phys. A 16 (2001) 822 [arXiv:hep-th/0007018]; "Towards the large N limit of pure N = 1 super Yang Mills," Phys. Rev. Lett. 86 (2001) 588 [arXiv:hep-th/0008001]. 